\title{
ÜBER DAS VERHALTEN DER ANALYTISCHEN ABBILDUNGEN RIEMANNSCHER FLÄCHEN AUF DEM IDEALEN RAND VON MARTIN
}

\author{
C. CONSTANTINESCU und A. CORNEA
}

Es sei $f$ eine meromorphe nichtkonstante Funktion im Kreise $|z|<1$. Die Theorie des Verhaltens von $f$ auf dem Rande des Kreises wird von $z$ wei bekannten Sätzen beherrscht: der Satz von Riesz-Lusin-Priwaloff-FrostmanNevanlinna, auf Grund dessen eine Menge aus $|z|=1$, für die die Winkelgrenzwerte von $f$ in einer Menge der Kapazität Null liegen, vom Lebesgueschen Masse Null ist und der Satz von Fatou-Nevanlinna, welcher besagt, dass, falls die Funktion $f$ beschränktartig ist, dann hat sie fast überall auf $|z|=1$ Winkelgrenzwerte. In vorliegender Arbeit werden diese zwei Sätze auf folgende Weise verallgemeinert. An Stelle des Kreises $|z|<1$ betrachten wir eine beliebige Riemannsche Fläche $R$ mit Greenscher Funktion, und an Stelle der meromorphen Funktion $f$ nehmen wir eine nichtkonstante analytische Abbildung von $R$ in einer Riemannschen Fläche $R^{\prime}\left(R^{\prime}\right.$ offen oder kompakt, mit oder ohne Greenscher Funktion). Es sei $\Delta$, bzw. $\Delta^{\prime}$, der ideale Rand von $R$, bzw. $R^{\prime}$, im Sinne von $\operatorname{Martin}^{11}$ [11]; wir werden im Abschnitt IV eine Teilmenge $\mathfrak{F}(f)$ der Menge $\Delta$ und eine Abbildung $\hat{f}$ von $\tilde{F}(f)$ in $R^{\prime} \cup \Delta^{\prime}$ definieren. Die Verallgemeinerung des Satzes von Fatou-Nevanlinna besteht in der Behauptung, dass, falls $f$ eine Lindelöfsche Abbildung ist [6], so ist $\Delta-\mathfrak{F}(f)$ von harmonischen Masse Null und die des Satzes von Riesz-Lusin-Priwaloff-Frostman-Nevanlinna darin, dass, wenn $\hat{f}(A)$ eine polare Menge auf $R^{\prime} \cup \Delta^{\prime}$ ist ${ }^{2)}$, so ist die Menge $A \subset \mathfrak{F}(f)$ vom harmonischen Masse Null. Ist $R$ der Kreis $|z|<1$ und $R^{\prime}$ die Riemannsche Kugel $|w| \leqq \infty$, so fallen, wie bekannt ist, die Begriffe "beschränktartige Funktion" und "Lindelöfsche Abbildung" zusammen; die polaren Mengen auf

Received October 30, 1959.

1) Hat $R^{\prime}$ keine Greensche Funktion, so definieren wir $\Delta^{\prime}$ in Bezug auf $R^{\prime}-G^{\prime}$, wo $G^{\prime}$ eine Kreisscheibe auf $R^{\prime}$ ist; der so erhaltene ideale Rand hängt von $G^{\prime}$ nicht ab. Ist $R^{\prime}$ kompakt, so ist $\Delta^{\prime}$ leer.

2) Eine polare Menge auf $R^{\prime} \cup \Delta^{\prime}$ ist eine Menge der Kapazität Null auf $R^{\prime}$ und vom harmonischen Masse Null auf $\Delta^{\prime}$. 
$R^{\prime}$ sind gerade die Mengen der Kapazität Null und $\Delta$ ist die Menge $|z|=1$. Es sei $\mathfrak{F}^{*}(f)$ die Menge der Punkte $e^{i \theta}$, für die $f$ einen Winkelgrenzwert besitzt und

$$
f^{*}\left(e^{i \theta}\right)=\lim _{z \rightarrow \ngtr e^{i \theta}} f(z) \quad\left(e^{i \theta} \in \mathfrak{F}^{*}(f)\right) .
$$

Da wir beweisen werden, dass, bis auf eine Menge vom Lebesgueschen Masse Null, $\mathfrak{F}^{*}(f) \subset \widetilde{F}(f)$ ist und $\hat{f}$ mit $f^{*}$ fast überall auf $\mathfrak{F}^{*}(f) \cap \mathfrak{F}(f)$ gleich sind, so folgt sofort aus der hier angegebener Verallgemeinerung der Satz von RieszLusin-Priwaloff-Frostman-Nevanlinna. Ist $f$ beschränktartig (und sogar in allgemeineren Fällen), so wird sich ergeben, dass $\mathfrak{F}(f)$ und $\mathfrak{F}^{*}(f)$, bis auf eine Menge vom Lebesgueschen Masse Null, gleich sind. Das erlaubt uns aus der allgemeineren Behauptung, dass $\Delta-\mathfrak{F}(f)$ vom harmonischen Masse Null ist, den klassischen Satz von Fatou-Nevanlinna zu folgern.

Es sei jetzt $|f(z)|<1$ in $|z|<1, f(0)=0, A$ eine Borelsche Menge aus $\mathfrak{F}^{*}(f)$ und $f^{*}(A)$ eine Menge auf $|w|=1$. Nach dem Lemma von Löwner [16] ist das Mass von $f^{*}(A)$ nicht kleiner als das der Menge $A$. Auch dieser Satz findet eine Verallgemeinerung mittels der oben eingeführten Begriffe.

Die ersten zwei Sätze finden einige Anwendungen in der Theorie der Klassifikation der Riemannschen Flächen.

Die wesentlichen Ergebnisse dieser Arbeit sind in einer Note [2] publiziert worden.

In den ersten zwei Anschnitten führen wir zwei Operatore $E_{f}$ und $I_{f}$ ein, die mit der Abbildung $f$ verknüpft sind; falls $R$ eine Teilmenge von $R^{\prime}$ und $f$ die identische Abbildung von $R$ in $R^{\prime}$ ist, so fallen die Operatore $E_{f}$ und $I_{f}$ mit den Extremisierungs-und Inextremisierungsoperatoren zusammen [9], [5], [1]. Da diese Operatore auch ein selbständiges Interesse aufweisen, haben wir ihre Theorie etwas mehr entwickelt, als das für den Beweis der obenerwähnten Sätze unbedingt notwendig war. In III untersuchen wir den Zusammenhang zwischen diesen Operatoren und den idealen Rand von Martin. Im Abschnitt IV beweisen wir die Fundamentalsätze und in V zeigen wir, wie die klassischen Sätze aus ihnen abgeleitet werden können. Die letzten Abschnitte sind einigen Anwendungen gewidmet.

\section{Der Operator $E_{f}$}

Wir werden folgende Tatsachen und Bezeichnungen benutzen. Es sei $G$ 
eine offene Menge auf einer nicht umbedingt zusammenhängenden Riemannschen Fläche $R$ derart, dass jede Komponente von $G$ eine Greensche Funktion besitzt, und $\psi$ eine "Randfunktion" von $G$, d.h. eine reelle Funktion auf dem relativen Rande von $G$ (wobei die Werte $\pm \infty$ nicht ausgeschlossen sind). Wir bezeichnen mit $H_{\tilde{G}}^{\psi}$ die normierte Lösung des Dirichletschen Problems auf $G$ mit $\psi$ als Randfunktion (falls diese existiert) [13]. Darunter versteht man

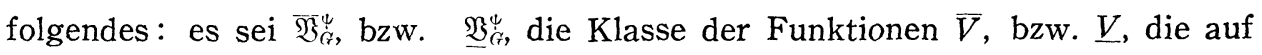
$G$ superharmonisch und nach unten beschränkt sind, bzw. subharmonisch und nach oben beschränkt sind, ${ }^{3)}$ und welche noch folgende Bedingungen erfüllen:

$$
\lim _{q \rightarrow p} \bar{V}(q) \geq \psi(p), \text { bzw. } \varlimsup_{q \rightarrow p} \underline{V}(q) \leq \psi(p)
$$

für jeden Punkt $p \in F r G$, bis auf eine Menge der Kapazität Null,') und für jede nichtkompakte Folge $\left\{q_{n}\right\}$ auf $G$ ist

$$
\lim _{n \rightarrow \infty} \vec{V}\left(q_{n}\right) \geq 0, \quad \text { bzw. } \lim _{n \rightarrow \infty} \underline{V}\left(g_{n}\right) \leq 0 .
$$

Man bezeichnet

$$
\begin{aligned}
& \bar{H}_{\dot{G}}^{\psi}(p)=\inf \left\{\bar{V}(p) \mid \bar{V} \in \overline{\mathfrak{B}}_{\dot{G}}^{\psi}\right\} \\
& \underline{H}_{\tilde{G}}^{\psi}(p)=\sup \left\{\underline{V}(p) \mid \underline{V} \in \underline{\mathfrak{B}}_{G}^{\psi}\right\} .
\end{aligned}
$$

Sind diese zwei Funktionen gleich, so bezeichnet man sie mit $H_{\tilde{G}}^{\iota}$, und $\psi$ heisst eine lösbare Randfunktion; $H_{G}^{\psi}$ ist in jeder Komponente von $G$ entweder eine harmonische Funktion, oder identisch $\pm \infty$. Jede halbbeschränkte Borelsche Funktion ist lösbar. Ist $\left\{\psi_{n}\right\}$ eine nichtabnähmende Folge von nichtnegativen lösbaren Randfunktionen, die gegen die Randfunktion $\psi$ konvergieren, so ist $\psi$ lösbar und

$$
\lim _{n \rightarrow \infty} H_{\dot{G}}^{\psi}=H_{\dot{G}}^{\psi}
$$

Sind $\psi_{1}, \psi_{2}$ positiv und lösbar und $\alpha_{1}, \alpha_{2}$ positive Zahlen, so ist auch $\alpha_{1} \psi_{1}+\alpha_{2} \psi_{2}$ lösbar und

$$
H_{G}^{\alpha_{1} \psi_{1}+\alpha_{2} \psi_{2}}=\alpha_{1} H_{G}^{\psi_{1}}+\alpha_{2} H_{\dot{G}}^{\psi_{2}^{2}} .
$$

Ist $S$ superharmonisch und nichtnegativ auf $R$, so ist $H_{G}^{S} \leq S$ auf $G$. Ist $G_{1} \subset G_{2}$, so ist $H_{G_{2}}^{S} \leq H_{G_{1}}^{S}$ auf $G_{1}$.

3) Wir lassen zu, dass eine superharmonische Funktion identisch $+\infty$ in einer oder mehreren Komponenten von $G$ ist. Dasselbe für subharmonische Funktionen und $-\infty$.

4' d.h. ihr Durchschnitt mit jeder Kreisscheibe ist eine Menge der Kapazität Null auf dieser Kreisscheibe. 
Es sei $F$ eine abgeschlossene Menge auf $R$, derart dass jede Komponente von $R-F$ eine Greensche Funktion besitzt, und $S$ eine positive superharmonische Funktion auf $R$. Wir bezeichnen nach Martin [11] mit $S_{F}$ die Funktion, die auf $F$ gleich $S$ und auf $R-F$ gleich $H_{R-F}^{S}$ ist; weiter sei $S_{F}^{*}$ die Funktion

$$
S_{F}^{*}(p)=\lim _{q \rightarrow p} S_{F}(q) .
$$

$S_{F}^{*}$ ist eine nichtnegative superharmonische Funktion auf $R$ [11].

Es sei $u$ eine nichtnegative harmonische Funktion auf $R$, und $S_{1}, S_{2}$ zwei nichtnegative superharmonische Funktionen auf $R$, für die $u \leq S_{1}+S_{2}$ ist. Dann kann man zwei harmonische Funktionen $u_{1}, u_{2}$ auf $R$ konstruieren ([8] Hilfssatz von Kjellberg), derart dass

$$
u=u_{1}+u_{2}, \quad 0 \leq u_{i} \leq S_{i} \quad(i=1,2)
$$

ist.

Für zwei superharmonische (bzw. subharmonische) Funktionen $S_{1}, S_{2}$ auf $R$ werden wir mit $S_{1} \vee S_{2}$ (bzw. $S_{1} \wedge S_{2}$ ) die Funktion auf $R$ bezeichnen, die durch folgende Beziehung definiert ist:

$S_{1} \vee S_{2}(p)=\inf \left\{S(p) \mid S\right.$ superharmonisch auf $\left.R, S_{1} \leq S, S_{2} \leq S\right\}$

(bzw. $S_{1} \wedge S_{2}(p)=\sup \left\{S(p) \mid S\right.$ subharmonisch auf $\left.R, S_{1} \geq S, S_{2} \geq S\right\}$ ).

Es sei $S_{0}$ die Funktion

$$
S_{0}(p)=\lim _{q \rightarrow p} S_{1} \vee S_{2}(q) .
$$

Sie sind offenbar nach unten halbstetig und nicht grösser als $S_{1} \vee S_{2}$. Es sei $G$ eine Kreisscheibe auf $R$ und $S$ eine superharmonische Funktion auf $R$ für die $S_{i} \leq S(i=1,2)$ ist. Dann ist auf $G$

$$
H_{G}^{s_{0}} \leq H_{G}^{s} \leq S \quad \text { und } \quad H_{G}^{S_{0}}(q) \leq S_{1} \vee S_{2}(q)
$$

für $q \in G$. Da $H_{G}^{S_{0}}$ stetig ist, so ist auch $H_{G}^{S_{0}} \leq S_{0}$, woraus man erkennt, dass $S_{0}$ superharmonisch ist. Da $S_{i} \leq S_{1} \vee S_{2}$ und $S_{i}$ halbstetig nach unten ist, so ist auch $S_{i} \leq S_{0}$ und somit, laut der Definition von $S_{1} \vee S_{2}$,

$$
S_{1} \vee S_{2} \leq S_{0} .
$$

Daraus folgt, dass $S_{1} \vee S_{2}$ gleich $S_{0}$ und deshalb superharmonisch ist. Ähnlicherweise beweist man, dass $S_{1} \wedge S_{2}$ (für $S_{1}, S_{2}$ subharmonische Funktionen) subharmonisch ist. Für $\alpha \leq 0$ ist 


$$
\alpha\left(S_{1} \vee S_{2}\right)=\left(\alpha S_{1}\right) \wedge\left(\alpha S_{2}\right)
$$

Es seien $u_{1}, u_{2}$ harmonische Funktionen. Dann ist

$$
u_{1}+u_{2}=\max \left(u_{1}, u_{2}\right)+\min \left(u_{1}, u_{2}\right) .
$$

Es seien $\left\{R_{\ell}\right\}_{\iota \in J}$ die Komponenten von $R,\left\{R_{\iota n}\right\}_{n}$ eine normale Ausschöpfung von $R_{\iota}([14]$, Seite 25$)$ und $R_{n}=\bigcup_{\iota \in J} R_{\iota n}$. Dann ist

$$
u_{1} \vee u_{2}=\lim _{n \rightarrow \infty} H_{R_{n}}^{\max \left(u_{1}, n_{2}\right)}, \quad u_{1} \wedge u_{2}=\lim _{n \rightarrow \infty} H_{R_{n}}^{\min \left(u_{1}, u_{2}\right)},
$$

und

$$
H_{R_{n}}^{\max \left(u_{1}, u_{2}\right)}+H_{R_{n}}^{\min \left(u_{1}, u_{2}\right)}=u_{1}+u_{2} .
$$

Hieraus ersieht man, dass, falls $u_{1} \vee u_{2}<+\infty$ auf $R$ ist, ${ }^{5)}$ so ist auch $u_{1} \wedge u_{2}$ $>-\infty$ auf $R$ und umgekehrt. In diesem Fall ist

$$
u_{1}+u_{2}=\left(u_{1} \vee u_{2}\right)+\left(u_{1} \wedge u_{2}\right)
$$

Mit $H P(R)$ (bzw. $S P(R)$ ) werden wir die Klasse der nichtnegativen harmonischen (bzw. superharmonischen ${ }^{6}$ ) Funktionen auf $R$ bezeichnen. Ist $R \in O_{G}$, so enthält $S P(R)$ nur konstante Funktionen und umgekehrt.

In der ganzen Arbeit sollen $R$ und $R^{\prime}$ zwei beliebige Riemannsche Flächen und $f$ eine nichtkonstante analytische Abbildung von $R$ in $R^{\prime}$ sein.

Wir werden in den ersten zwei Abschnitten nicht annehmen, dass die Riemannschen Flächen $R$ und $R^{\prime}$ zusammenhängend sind. Wir wollen nähmlich den Fall, wo $R$ eine offene (nicht zusammenhängende) Menge auf $R^{\prime}$ und $f$ die identische Abbildung ist, der in dieser Arbeit sehr oft vorkommen wird, in diese Betrachtungen einschliessen. Wie man sehen wird, spielt der Zusammenhang keine Rolle in Bezug auf den Operatoren $E_{f}$ und $I_{f}$, so dass die Beweise dadurch nicht komplizierter werden. Der Fall, wenn $R$ oder $R^{\prime}$ eine kompakte Komponente besitzen, muss für die Operatore $E_{f}$ und $I_{f}$ besonders betrachtet werden und, da dieser Fall trivial ist, werden wir der Einfachheit halber in den ersten zwei Abschnitten annehmen, dass keine der Komponente von $R$ oder $R^{\prime}$ kompakt ist.

5) d.h. $u_{1} \bigvee u_{2}(p) \neq+\infty$ für jedes $p \in R$.

6) Es ist erlaubt, dass $S \equiv+\infty$ auf einer oder mehreren Komponenten von $R$ ist, 
Definition. Für jedes $S \in S P(R)$ bezeichnen wir mit $E_{f} S$ die Funktion auf $R^{\prime}$, die durch folgende Beziehung definiert wird:

$$
E_{f} S\left(p^{\prime}\right)=\inf \left\{S^{\prime}\left(p^{\prime}\right) \mid S^{\prime} \circ f \geq S, S^{\prime} \in S P\left(R^{\prime}\right)\right\} \quad\left(p^{\prime} \in R^{\prime}\right) .
$$

Es sei $R_{\ell}^{\prime}$ eine Komponente von $R^{\prime}, R_{\iota}=f^{-1}\left(R_{\ell}^{\prime}\right) \neq \phi$ und $f_{\iota}$ die Abbildung von $R_{\iota}$ in $R_{\iota}^{\prime}$, die mit $f$ zusammenfällt. Es sei $S \in S P(R)$ und $S_{\iota}$ die Einschränkung von $S$ auf $R_{\iota}$; dann ist $E_{f} S$ gleich $E_{f_{\iota}} S_{\iota}$ auf $R_{\ell}^{\prime}$.

Ist $R$ eine offene Menge auf $R^{\prime}$ und $f$ die identische Abbildung, so werden wir auch $\underset{R}{E}$ an Stelle von $E_{f}$ setzen, da $f$ implizite bekannt ist. Falls keine Missverständnisse $z \mathrm{u}$ befürchten sind, so werden wir auch einfach $E$ schreiben.

Für jedes $S \in S P(R)$ werden wir mit $\bar{S}$ die Funktion auf $R^{\prime}$ bezeichnen, die folgendermassen definiert ist: ist $p^{\prime} \in f(R)$, so ist

$$
\bar{S}\left(p^{\prime}\right)=\sup \left\{S(p) \mid f(p)=p^{\prime}\right\} ;
$$

ist $p^{\prime} \in R^{\prime}-f(R)$, so ist $\bar{S}\left(p^{\prime}\right)$ Null. $\bar{S}$ ist nach unten halbstetig. In der Tat, sei $\left\{p_{n}^{\prime}\right\}$ eine Folge auf $R^{\prime}$ die gegen $p^{\prime} \in f(R)$ konvergiert, $p$ ein Punkt der sich in $p^{\prime}$ projeziert und $\left\{p_{n}\right\}$ eine Folge auf $R$, die gegen $p$ strebt und für die $f\left(p_{n}\right)=p_{n}^{\prime}$ ist. Dann ist

$$
\lim _{n \rightarrow \infty} \bar{S}\left(p_{n}^{\prime}\right) \geq \lim _{n \rightarrow \infty} S\left(p_{n}\right) \geq S(p) .
$$

Da $p$ beliebig war, so ist $\lim _{n \rightarrow \infty} \bar{S}\left(p_{n}^{\prime}\right) \geq \bar{S}\left(p^{\prime}\right)$. In den Punkten $p^{\prime} \in R^{\prime}-f(R)$ ist diese Beziehung offenbar. Die Ungleichungen

$$
S^{\prime} \circ f \geq S \quad \text { und } \quad S^{\prime} \geq \bar{S}
$$

sind offenbar äquivalent und deshalb ist

$$
E_{f} S\left(p^{\prime}\right)=\inf \left\{S^{\prime}\left(p^{\prime}\right) \mid S^{\prime} \geq \bar{S}, S^{\prime} \in S P\left(R^{\prime}\right)\right\} .
$$

SATZ 1. ES ist eine nichtnegative superharmonische Funktion und

$$
S \leq(E S) \circ f .
$$

Für $S_{1}, S_{2} \in S P(R)$ ist

$$
E\left(S_{1} \vee S_{2}\right)=E S_{1} \vee E S_{2} \quad \text { und } \quad E\left(S_{1}+S_{2}\right) \leq E S_{1}+E S_{2} .
$$

Wir werden mit $(E S)^{*}$ die Funktion

$$
(E S)^{*}\left(p^{\prime}\right)=\lim _{q^{\prime} \rightarrow p^{\prime}} E S\left(q^{\prime}\right)
$$


bezeichnen; sie ist offenbar nach unten halbstetig. Wir wollen zeigen, dass $(E S)^{*} \in S P\left(R^{\prime}\right)$ und daraus wird sich sofort ergeben, dass sie mit ES zusammenfällt. Es sei $S^{\prime} \geq \bar{S}, S^{\prime} \in S P\left(R^{\prime}\right)$ und $G^{\prime}$ eine Kreisscheibe auf $R^{\prime}$; auf dem Rande von $G^{\prime}$ ist $S^{\prime}$ nicht kleiner als $(E S)^{*}$ und deswegen ist $H_{G^{\prime}}^{(E S)^{*}} \leq S^{\prime}$ überall in $G^{\prime}$. Daraus folgt

$$
H_{G^{\prime}}^{(E S)^{*}} \leq E S \quad \text { und } \quad H_{G^{\prime}}^{(E S)^{*}} \leq(E S)^{*}
$$

in $G^{\prime}$, da $H_{G^{\prime}}^{(F S)^{*}}$ stetig ist. ( $\left.E S\right)^{*}$ ist somit eine superharmonische Funktion. Aus $\bar{S} \leq E S$ folgt, da $\bar{S}$ nach unten halbstetig ist, $\bar{S} \leq(E S)^{*}$. Deshalb ist

$$
E S \leq(E S)^{*}, \quad E S=(E S)^{*} .
$$

Damit ist der erste Teil des Satzes bewiesen. Die Subadditivität von $E$ folgt sofort aus der Definition. Aus $S_{i} \leq S_{1} \vee S_{2} \quad(i=1,2)$ folgt

$$
E S_{i} \leq E\left(S_{1} \vee S_{2}\right) \quad \text { und } \quad E S_{1} \vee E S_{2} \leq E\left(S_{1} \vee S_{2}\right)
$$

Da aber $S_{i} \leq\left(E S_{i}\right) \circ f \leq\left(E S_{1} \vee E S_{2}\right) \circ f$ ist, so ist auch

$$
S_{1} \vee S_{2} \leq\left(E S_{1} \vee E S_{2}\right) \circ f
$$

und

$$
E\left(S_{1} \vee S_{2}\right) \leq E S_{1} \vee E S_{2} .
$$

SATz 2. Es sei $R \stackrel{f}{\longrightarrow} R^{\prime} \stackrel{f}{\longrightarrow} R^{\prime \prime}$. Dann ist $E_{f^{\prime} \cdot f}=E_{f}, E_{f}$.

Wir bezeichnen $f^{\prime \prime}=f^{\prime} \circ f$. Für jedes $S \in S P(R)$ ist

$$
S \leq\left(E_{f} S\right) \circ f, \quad E_{f} S \leq\left(E_{f}, E_{f} S\right) \circ f^{\prime} .
$$

Daraus folgert man

$$
S \leq\left(E_{f}, E_{f} S\right) \circ f^{\prime \prime}, \quad E_{f^{\prime}} S \leq E_{f}, E_{f} S .
$$

Aus $S \leq\left(E_{f^{\prime \prime}} S\right) \circ f^{\prime \prime}=\left(\left(E_{f^{\prime \prime}} S\right) \circ f^{\prime}\right) \circ f$ folgt $E_{f} S \leq\left(E_{f^{\prime \prime}} S\right) \circ f^{\prime}$ und daher

$$
E_{f}, E_{f} S \leq E_{f \prime} S, \quad E_{f}, E_{f} S=E_{f^{\prime \prime}} S .
$$

SATz 3. Es sei $\left\{R_{n}^{\prime}\right\}$ eine nicht abnähmende Folge von offenen Mengen auf $R^{\prime}$, deren Vereinigung $R^{\prime}$ ist. Es sei $f_{n}$ die analytische Abbildung von $f^{-1}\left(R_{n}^{\prime}\right)$ in $R_{n}^{\prime}$ die mit $f$ zusammenfällt. Dann ist $E_{f_{n}} S \uparrow E_{f} S$ für jedes $S \in S P(R)$.

$E_{f_{n+1}} S$ ist superharmonisch und nichtnegativ auf $R_{n}^{\prime}$ und es ist $S \leq\left(E_{f_{n+1}} S\right) \circ f_{n}$ auf $f^{-1}\left(R_{n}^{\prime}\right)$. Daraus folgt $E_{f_{n}} \mathrm{~S} \leq E_{f_{n+1}} S$ auf $R_{n}^{\prime}$ und ähnlicherweise 
$E_{f_{n}} S \leq E_{f} S . \quad$ Es ist also

$$
\lim _{n \rightarrow \infty} E_{f_{n}} S \leq E_{f} S
$$

Die Funktion $\lim _{n \rightarrow \infty} E_{f_{n}} S$ ist nichtnegativ und superharmonisch auf $R^{\prime}$ und

$$
S \leq\left(\lim _{n \rightarrow \infty} E_{f_{n}} S\right) \circ f,
$$

woraus

$$
E_{f} S \leq \lim _{n \rightarrow \infty} E_{f_{n}} S
$$

folgt.

SATZ 4. Ist $S, S_{n} \in S P(R)(n=1,2, \ldots)$ und $S \leq \lim _{n \rightarrow \infty} S_{n}$, so ist auch

$$
E S \leq \lim _{n \rightarrow \infty} E S_{n} .
$$

Ist die Folge $\left\{S_{n}\right\}$ nichtabnehmend, so ist

$$
E\left(\lim _{n \rightarrow \infty} S_{n}\right)=\lim _{n \rightarrow \infty} E S_{n}
$$

Es seien $S^{\prime}, S^{*}$ die Funktionen

$$
S^{\prime}\left(p^{\prime}\right)=\lim _{n \rightarrow \infty} E S_{n}\left(p^{\prime}\right), \quad S^{*}\left(p^{\prime}\right)=\lim _{q^{\prime} \rightarrow p^{\prime}} S^{\prime}\left(q^{\prime}\right) .
$$

Man erkennt wie im Satz 1, dass $S^{*}$ superharmonisch ist. Aus

$$
S^{\prime}(f(p))=\lim _{n \rightarrow \infty} E S_{n}(f(p)) \geq \lim _{n \rightarrow \infty} S_{n}(p) \geq S(p)
$$

folgt $S^{\prime} \geq \bar{S}$ und, da $\bar{S}$ nach unten halbstetig ist, $S^{*} \geq \bar{S}$. Es ist also

$$
E S \leq S^{*} \leq S^{\prime}=\lim _{n \rightarrow \infty} E S_{n}
$$

Ist die Folge $\left\{S_{n}\right\}$ nicht abnähmend, so ist $\left\{E S_{n}\right\}$ auch nicht abnehmend und

$$
\lim _{n \rightarrow \infty} E S_{n} \leq E \lim _{n \rightarrow \infty} S_{n}
$$

Nach dem ersten Teil des Satzes ist aber

$$
E \lim _{n \rightarrow \infty} S_{n} \leq \lim _{n \rightarrow \infty} E S_{n},
$$

womit der Satz vollständig bewiesen ist.

Hilfssatz 1. Es sei $S \in S P(R)$ und $G^{\prime}$ eine offene Menge auf $R^{\prime}$, für die alle Komponenten eine Greensche Funktion besitzen. Ist $S_{R-f^{-1}\left(G^{\prime}\right)}^{*}=S$, so ist 


$$
(E S)_{R^{\prime}-G^{\prime}}^{*}=E S \text {. }
$$

Ist also ES nicht identisch unendlich auf einer Komponente $R_{f}^{\prime}$ von $R^{\prime}$, so ist ES harmonisch auf $G^{\prime} \cap R_{\iota}^{\prime}$.

Es sei $\bar{V}^{\prime} \in \overline{\mathfrak{V}}_{G^{\prime}}^{\bar{s}}$; dann ist offenbar $\bar{V}^{\prime} \circ f \in \overline{\mathfrak{V}}_{f^{-1}\left(G^{\prime}\right)}^{s}$. Daraus folgt sofort

$$
\overline{H_{f^{\prime}}^{s}}, \bar{f} \geq H_{f^{-1}\left(G^{\prime}\right)}^{s}=S, \quad \bar{S} \leq H_{G^{\prime}}^{\bar{s}} .
$$

Aus $\bar{S} \leq E S$ folgert man

$$
\bar{S} \leq \overline{H_{G^{\prime}}^{s}} \leq H_{\bar{G}^{\prime}}^{E S} \leq E S
$$

auf $G^{\prime} . \quad$ Da $\bar{S}$ nach unten halbstetig ist, so ist $\bar{S} \leq(E S)_{R^{\prime}-G^{\prime}}^{*} \leq E S$. Daraus erhalten wir

$$
(E S)_{R^{\prime}-G^{\prime}}^{*}=E S \text {, }
$$

da $(E S)_{R^{\prime}-G^{\prime}}^{*} \in S P\left(R^{\prime}\right)$ ist, was $z u$ beweisen war.

Wir bezeichnen mit $H P(f)$ die Klasse der Funktionen $u$ aus $H P(R)$, für welche, für jedes relativkompaktes Gebiet $G^{\prime}$ auf $R^{\prime}$,

$$
H_{\left.f-1, G^{\prime}\right)}^{u}=u
$$

auf $f^{-1}\left(G^{\prime}\right)$ ist. Diese Beziehung ist mit $u_{R-f^{-1}\left(G^{\prime}\right)}^{*}=u$ äquivalent. Die Klasse $H P(f)$ besitzt folgende Eigenschaften.

a) Ist $u \leq v$ und $u \in H P(R), v \in H P(f)$, so ist auch $u \in H P(f)$.

b) Ist $u, v \in H P(f)$ und sind $\alpha, \beta$ reelle nichtnegative Zahlen, so ist $\alpha u+\beta v \in H P(f)$.

c) Ist $u, v \in H P(f)$, so ist $u \wedge v, u \vee v \in H P(f)$.

d) Ist $\left\{u_{n}\right\}$ eine Folge aus $H P(f)$, die gegen u konvergiert und gleichmässig von einer Funktion $u_{0} \in H P(R)$ majoriert wird, so ist $u \in H P(f)$.

Es sei $G^{\prime}$ ein relativkompaktes Gebiet auf $R^{\prime}$.

a) Aus

$$
v=H_{f^{-1}\left(G^{\prime}\right)}^{v}=H_{f^{-1}\left(G^{\prime}\right)}^{u}+H_{f^{-1}\left(G^{\prime}\right)}^{v-u} \leq H_{f^{-1}\left(G^{\prime}\right)}^{u}+v-u
$$

erhalten wir

$$
u \leq H_{f^{-1}\left(G^{\prime}\right)}^{u}, \quad u=H_{f^{-1}\left(G^{\prime}\right)}^{u} .
$$

b) folgt sofort aus

$$
H_{f^{-1}\left(G^{\prime}\right)}^{\alpha u+\beta v}=\alpha H_{f^{-1}\left(G^{\prime}\right)}^{u}+\beta H_{f^{-1}\left(G^{\prime}\right)}^{v} .
$$

c) folgt aus $0 \leq u \wedge v \leq u \vee v \leq u+v$ und aus a) und b). 
d) Wir setzen

$$
v_{n}=u_{1} \vee u_{2} \vee \cdots \vee u_{n}
$$

$\left\{v_{n}\right\}$ ist eine nichtabnehmende Folge aus $H P(f)$, die gegen eine harmonische Funktion $v \leq u_{0}$ konvergiert. Wir haben

$$
H_{f^{-1}\left(G^{\prime}\right)}^{v}=\lim _{n \rightarrow \infty} H_{f^{-1}\left(G^{\prime}\right)}^{v_{n}}=\lim _{n \rightarrow \infty} v_{n}=v .
$$

Aus $u \leq v \in H P(f)$ und a) folgt $u \in H P(f)$.

SATZ 5. Ist $u \in H P(f)$, so ist Eu auf jeder Komponente von $R^{\prime}$ entweder harmonisch, oder identisch unendlich.

Dieser Satz folgt unmittelbar aus dem Hilfssatz 1 und aus der Definition von $H P(f)$.

Es sei $G$ eine offene Menge auf $R$, so dass jede Komponente von $G$ eine Greensche Funktion besitzt. Wir bezeichnen mit $\mathfrak{u}_{G}$ die Klasse der Funktionen $u \in H P(G)$ für die, für alle Punkte $q$ des relativen Randes von $G$,

$$
\varlimsup_{p \rightarrow q} u(p)<\infty
$$

ist und

$$
\varlimsup_{p \rightarrow q} u(p)=0
$$

ist, für alle Punkte $q$ des relativen Randes von $G$, die für das Dirichletsche Problem regulär sind. Für eine Funktion $u \in H P(G)$ bezeichnen wir mit $\bar{u}$ die Funktion auf $R$ die auf $G$ gleich $u$ und auf $R-G$ Null ist.

Satz 6. Es sei $\eta$ die identische Abbildung von $G$ in $R$.

a) Es sei $u \in \mathfrak{U}_{G}$ und $D$ eine relativkompakte offene Menge auf $R$; dann ist

$$
\bar{u} \leq H_{D}^{\bar{u}}
$$

auf $D$.

b) $\mathfrak{H}_{G} \subset H P(\eta)$.

c) Ist $u \in H P(\eta)$ und

$$
\lim _{p \rightarrow q} u(p)<\infty
$$

für alle Punkte $q$ des relativen Randes von $G$, so ist $u \in \mathfrak{H}_{G}$.

d) Der Operator $E_{\eta}$ ist auf $H P(\eta)$ linear. 
e) Es sei $\left\{u_{n}\right\}$ eine nicht zunehmende Folge aus $H P(\eta)$, derart dass

$$
E_{\eta} u_{n_{0}}<\infty
$$

für ein $n_{0}$ ist. Dann ist $\lim _{n \rightarrow \infty} E_{\eta} u_{n}=E_{\eta}\left(\lim _{n \rightarrow \infty} u_{n}\right)$.

a) Es genügt die Ungleichung auf $G \cap D$ zu beweisen. Aus $\bar{u} \in \underline{\mathfrak{B}}_{\text {rn }}^{\bar{u}}$ und $H_{D}^{\bar{u}} \in \overline{\mathfrak{B}}_{G \cap D}^{\bar{u}}$ folgt $\bar{u} \leq H_{D}^{\bar{u}}$ auf $G \cap D$.

b) Es sei $u \in \mathfrak{H}_{a}$ und $D$ ein relativkompaktes Gebiet auf $R$. Dann ist $\overline{\mathfrak{V}}_{G \cap D^{\circ} \eta}^{\bar{u}}=\overline{\mathfrak{V}}_{\eta-1(D)}^{u}$ und deshalb ist

$$
H_{G \cap D^{\circ} \eta}^{\bar{u}}=H_{\eta_{-1}(D)}^{u} .
$$

Nach a) ist

$$
u=\bar{u} \circ \eta \leq H_{u \cap D}^{\bar{u}} \circ \eta=H_{\eta-1(D)}^{u}
$$

und somit $u \in H P(\eta)$.

c) Es ist nur zu beweisen, dass

$$
\lim _{p \rightarrow q} u(p)=0
$$

ist, wo $q$ ein regulärer Punkt des relativen Randes von $G$ ist. Es sei $D$ eine Kreisscheibe, die den Punkt $q$ enthält. Nach dem Beweis von b) ist

$$
u=H_{\eta-1(D)}^{u}=H_{G \cap D}^{\bar{u}} \circ \eta \text {. }
$$

Daraus, aus der Regularität von $q$, aus $\bar{u}(q)=0$ und aus der Stetigkeit von $\bar{u}$ in $q$ folgt sofort die obige Beziehung.

d) Es genügt den Beweis im Falle dass $R$ zusammenhängend ist durchzuführen. Es sei $u_{1}, u_{2} \in H P(\eta)$. Ist $E u_{i}(i=1,2)$ identisch unendlich für wenigstens ein $i$, so ist evident

$$
E\left(u_{1}+u_{2}\right)=E u_{1}+E u_{2} .
$$

Wir können also nach Satz 5 annehmen, dass jede Funktion $E u_{i}$ harmonisch ist. Da $u_{i} \leq E u_{i} \circ \eta$ ist, so folgt aus c) $u_{i} \in \mathfrak{U}_{G}$. Es sei $\left\{R_{n}\right\}$ eine normale Ausschöpfung von $R$. Sei $\eta_{n}$ die identische Abbildung von $\eta^{-1}\left(R_{n}\right)$ in $R_{n}$. Nach a) ist $\bar{u}_{i} \leq H_{R_{i} \text {. }}^{\bar{u}_{i}}$ Daraus folgt

$$
E_{\tau_{n}} u_{i} \leq H_{B_{n} \cdot}^{\bar{u}_{i}}
$$

Da aber $E_{\eta_{n}} u_{i} \in \overline{\mathfrak{V}}_{R_{n}}^{\overline{u_{i}}}$, so ist

$$
E_{r_{n}} u_{i}=H_{R_{n}}^{\bar{u}_{i}}
$$


Ähnlicherweise zeigt man, dass $E_{\eta_{n}}\left(u_{1}+u_{2}\right)=H_{R_{n}}^{\bar{u}_{1}+\bar{u}_{2}}$ ist. Daraus ergibt sich

$$
E_{\eta_{n}}\left(u_{1}+u_{2}\right)=E_{\eta_{n}} u_{1}+E_{\eta_{n}} u_{2}
$$

und für $n \rightarrow \infty$ folgt nach Satz 3

$$
E_{\eta}\left(u_{1}+u_{2}\right)=E_{\eta} u_{1}+E_{\eta} u_{2} .
$$

e) Wir bezeichnen $v_{n}=u_{n_{0}}-u_{n}\left(n>n_{0}\right)$. Dann $\left\{v_{n}\right\}$ ist eine nicht abnähmende gleichmässig beschränkte Folge aus $H P(\eta)$ und nach d) und Satz 4 ist

$$
\begin{aligned}
& \lim _{n \rightarrow \infty} E u_{n}=\lim _{n \rightarrow \infty} E\left(u_{n_{0}}-v_{n}\right)=E u_{n_{0}}-\lim _{n \rightarrow \infty} E v_{n}= \\
& =E u_{n_{0}}-E\left(\lim _{n \rightarrow \infty} v_{n}\right)=E u_{n_{0}}-E\left(u_{n_{0}}-\lim _{n \rightarrow \infty} u_{n}\right)=E\left(\lim _{n \rightarrow \infty} u_{n}\right) .
\end{aligned}
$$

Es sei $R$ zusammenhängend ${ }^{7)}$. Eine Funktion $u$ aus $H P(R)$ heisst singulär, wenn jede beschränkte nichtnegative harmonische Funktion, die kleiner als $u$ ist, identisch verschwindet. Die Funktion heisst quasibeschränkt, falls sie Grenzfunktion einer nichtabnehmenden Folge von beschränkten harmonischen Funktionen ist. Ist $v$ quasibeschränkt und $w$ singulär, so ist $v \wedge w=0$. Parreau hat beweisen, dass jede Funktion $u \in H P(R)$ eindeutig als Summe einer quasibeschränkten und einer singulären Funktion darstellbar ist [13]. Es sei $\alpha$ eine positive Zahl. Dann ist

$$
u-\alpha \leq(u-\alpha) \vee 0 \leq u, \quad 0 \leq u-(u-\alpha) \vee 0 \leq \alpha .
$$

Ist $u$ singulär, so ist

$$
0 \leq u-(u-\alpha) \vee 0 \leq \alpha \wedge u \leq u \text { und }(u-\alpha) \vee 0=u .
$$

Die Funktion $u \in H P(R) \quad(u \neq 0)$ heisst minimal, wenn aus $v \in H P(R)$ und $v \leq u$,

$$
v=\alpha u
$$

folgt, wo $\alpha$ eine reelle Zahl ist [11]. Für zwei nichtproportionale minimale Funktionen $u, v$ ist $u \wedge v=0$.

Eine Funktion $u \in H P(R)$ heisst eine diskrete Funktion, wenn sie als endliche oder abzählbare Summe von minimalen Funktionen darstellbar ist; $u$ heisst total nichtdiskrete Funktion, falls es keine minimale Funktion gibt, die

7) Auch für die weitere Betrachtungen ist der Zusammenhang von $R$ nicht notwendig, aber sie sind nur für $R$ zusammenhängend interessant. 
kleiner als $u$ ist. Jede Funktion $u \in H P(R)$ ist eindeutig als Summe einer diskreten und einer total nichtdiskreten Funktion darstellbar. In der Tat, es sei $\left\{K_{s}\right\}$ die Klasse aller minimalen Funktionen auf $R$, die in einem Punkt gleich 1 sind und $\alpha(s)$ die grösste reelle Zahl für die $\alpha(s) K_{s} \leq u$ ist. Da $K_{s} \wedge K_{s^{\prime}}=0$ für $s \neq s^{\prime}$ ist, so ist ([5], Theorem 2.2)

$$
\sum_{i=1}^{n} \alpha\left(s_{i}\right) K_{s_{i}}=\bigvee_{i=1}^{n} \alpha\left(s_{i}\right) K_{s_{i}} \leq u
$$

Daraus folgt, dass $\alpha(s)$ nur für abzählbar vielen $s$ positiv ist und es gilt $\sum_{\alpha(s)>0} \alpha(s) K_{s} \leq u$.

$$
u=\sum_{\alpha(s)>0} \alpha(s) K_{s}+\left(u-\sum_{\alpha(s)>0} \alpha(s) K_{s}\right)
$$

ist offenbar die gesuchte Darstellung.

$u \in H P(R)$ heisst ein harmonisches Mass [5], [7], wenn

$$
u \leq 1, \quad u \wedge(1-u)=0
$$

ist. Jede minimale beschränkte Funktion $u$, für die sup $u=1$ ist, ist ein harmonisches Mass. Jedes harmonische Mass $u$ besitzt die Eigenschaft

$$
\frac{u-\alpha}{1-\alpha} \vee 0=u \quad(0<\alpha<1)
$$

Da $u$ nicht grösser als 1 ist, ist nähmlich

$$
\frac{u-\alpha}{1-\alpha} \leq u \text { und } \frac{u-\alpha}{1-\alpha} \vee 0 \leq u
$$

Es sei $v=u-\frac{u-\alpha}{1-\alpha} \vee 0 \leq u$. Dann ist

$$
0 \leq v \leq u-\frac{u-\alpha}{1-\alpha}=\frac{\alpha(1-u)}{1-\alpha}
$$

und

$$
0 \leq v \leq \frac{\alpha(1-u)}{1-\alpha} \wedge u \leq \max \left(1, \frac{\alpha}{1-\alpha}\right) \cdot((1-u) \wedge u)=0
$$

Umgekehrt, besitzt $u \in H P(R) \quad(u \leq 1)$ die Eigenschaft $(M)$, so ist $u$ ein harmonisches Mass. Laut (2) ist nähmlich

$$
\left(\frac{u-\alpha}{1-\alpha} \vee 0\right)+\left(\frac{u-\alpha}{1-\alpha} \wedge 0\right)=\frac{u-\alpha}{1-\alpha}
$$

und somit 


$$
\begin{aligned}
& \frac{u-\alpha}{1-\alpha} \wedge 0=\frac{u-\alpha}{1-\alpha}-u=-\frac{\alpha(1-u)}{1-\alpha}, \\
& -\frac{1}{\alpha}((u-\alpha) \wedge 0)=1-u .
\end{aligned}
$$

Nach (1) ist dann auch

$$
\frac{\alpha-u}{\alpha} \vee 0=-\frac{1}{\alpha}((u-\alpha) \wedge 0)=1-u
$$

Es sei $\left\{R_{n}\right\}$ eine normale Ausschöpfung von $R$. Dann ist offenbar

$$
(1-u) \vee u \geq H_{R_{n}}^{\left(\frac{u-\alpha}{1-\alpha}\right)^{+}}+H_{R_{n}}^{\left(\frac{\alpha-u}{\alpha}\right)^{+}},
$$

wo $\varphi^{+}=\max (\varphi, 0)$ gesetzt wurde. Für $n \rightarrow \infty$ erhalten wir

$$
(1-u) \vee u \geq \frac{u-\alpha}{1-\alpha} \vee 0+\frac{\alpha-u}{\alpha} \vee 0=u+(1-u)=1
$$

und, nach (2), $(1-u) \wedge u=0$.

Aus der Eigenschaft $(M)$ folgt unmittelber, dass für ein harmonisches Mass $u$

$$
\sup u=1 \quad \text { oder } \quad 0
$$

ist.

Die harmonischen Masse (bzw. singulären Funktionen) besitzen noch eine wichtige Eigenschaft. Für jedes $\alpha<1$ (bzw. $\alpha<\infty$ ) ist

$$
H_{\{u<\alpha\}}^{u}=u \text {. }
$$

In der Tat, die Funktion

$$
S=\left\{\begin{array}{cc}
H_{\{u<\alpha\}}^{u} & \text { in }\{u<\alpha\} \\
u & \text { in }\{u \geq \alpha\}
\end{array}\right.
$$

ist superharmonisch und

$$
\left.\frac{u-\alpha}{1-\alpha} \leq S \leq u \quad \text { (bzw. } u-\alpha \leq S \leq u\right),
$$

woraus

$$
\left.u=\frac{u-\alpha}{1-\alpha} \vee 0 \leq S \leq u \quad \text { (bzw. } u=(u-\alpha) \vee 0 \leq S \leq u\right)
$$

folgt.

SATz 7. Es sei u eine minimale Funktion auf $R$. Dann können nur folgende Fälle vorkommen: 
a) $E u \equiv \infty$,

b) $E u=\alpha g^{\prime} p^{\prime}$,

c) Eu ist eine minimale Funktion.

Dabei ist $g_{p^{\prime}}^{\prime}$ die Greensche Funktion von $R^{\prime}$ und $\alpha$ eine positive Zahl. Ist $u$ minimal beschränkt, so kommt nur der Fall c) vor, und Eu ist auch minimal beschränkt.

Wir nehmen an, dass $E u$ nicht identisch unendlich ist und es seien $S_{1}^{\prime}$, $S_{2}^{\prime} \in S P\left(R^{\prime}\right)$, derart dass keine identisch Null ist und $E u=S_{1}^{\prime}+S_{2}^{\prime}$. Dann ist $u \leq S_{1}^{\prime} \circ f+S_{2}^{\prime} \circ f$ und nach Kjellbergschem Hilfssatz gibt es zwei harmonische Funktionen $u_{1}, u_{2}$, derart dass $u=u_{1}+u_{2}, 0 \leq u_{i} \leq S_{i}^{\prime} \circ f(i=1,2)$ ist. Da $u$ minimal ist, so haben wir

$$
u_{i}=\alpha_{i} u\left(0 \leq \alpha_{i} \leq 1\right) \text { und } \alpha_{1}+\alpha_{2}=1 .
$$

Keine der Zahlen $\alpha_{1}, \alpha_{2}$ ist Null, denn wäre z.B. $\alpha_{1}=0$, so ist $u=u_{2} \leq S_{2}^{\prime} \circ f$, woraus

$$
E u=S_{2}^{\prime}, \quad S_{1}^{\prime}=0
$$

folgen würde, entgegen der Voraussetzung über $S_{1}^{\prime}$. Es ist also $u=\frac{1}{\alpha_{i}} u_{i}$ $\leq \frac{1}{\alpha_{i}} S_{i}^{\prime} \circ f$ und somit $E u \leq \frac{1}{\alpha_{i}} S_{i}^{\prime}$. Daraus folgt

$$
E u=\alpha_{1} E u+\alpha_{2} E u \leq S_{1}^{\prime}+S_{2}^{\prime}=E u,
$$

woraus man erkennt, dass $\alpha_{i} E u=S_{i}^{\prime}$ für jedes $i$ ist. Es ist somit $\frac{S_{1}^{\prime}}{\alpha_{1}}=\frac{S_{2}^{\prime}}{\alpha_{2}}$. Nach dem Satz von Riesz, kann man Eu in der Form

$$
E u=P^{\prime}+u^{\prime}
$$

darstellen, wo $P^{\prime}$ ein (mit Greenscher Funktion gebildeter) Potential und $\boldsymbol{u}^{\prime}$ eine nichtnegative harmonische Funktion ist. Wären beide nicht Null, so wären laut obigen Betrachtungen, $u^{\prime}$ und $P^{\prime}$ proportional, was widersinnig ist. $E u$ ist also entweder eine harmonische Funktion, oder ein Potential. Im ersten Falle ist sie minimal, denn ist $v^{\prime} \in H P\left(R^{\prime}\right)$ und $0<v^{\prime}<E u$, so setzen wir $S_{1}^{\prime}=v^{\prime}, S_{2}^{\prime}=E u-v^{\prime}$ und wir erhalten wie oben $v^{\prime}=\alpha E u$. Im zweiten Fall ist

$$
E u=\int_{R^{\prime}} g_{p^{\prime}}^{\prime} d \mu^{\prime}\left(p^{\prime}\right)
$$


Sind $A_{1}^{\prime}, A_{2}^{\prime}$ zwei punktfremde Borelsche Mengen auf $R^{\prime}$, deren Vereinigung $R^{\prime}$ ist, so hat höchstens eine von ihnen positives $\mu^{\prime}$ Mass; im entgegengesetzten Falle setzen wir

$$
S_{i}^{\prime}=\int_{A^{\prime} i} g^{\prime} p^{\prime} d \mu^{\prime}\left(p^{\prime}\right)>0 \quad(i=1,2) .
$$

Laut obiger Betrachtungen, müssen $S_{1}^{\prime}, S_{2}^{\prime}$ proportional sein, was unmöglich ist, wegen der Eindeutigkeit des Masses eines Potentials. Daraus folgt, dass ein Punkt $p^{\prime} \in R^{\prime}$ und eine reelle Zahl $\alpha$ existieren, derart dass

$$
\mu\left(A^{\prime}\right)=\left\{\begin{array}{l}
\alpha \text { für } p^{\prime} \in A^{\prime} \\
0 \text { für } p^{\prime} \notin A^{\prime}
\end{array}\right.
$$

und $E u=\alpha g^{\prime} p^{\prime}$ ist.

Ist $u$ beschränkt, so ist offenbar auch $E u$ beschränkt, was nur im Falle c) möglich ist.

In einer früheren Arbeit [1] haben wir mit $U$ die Klasse der Riemannschen Flächen mit Greenscher Funktion bezeichnet, die wenigstens eine beschränkte minimale Funktion besitzen. Aus dem Satz 7 folgt,

Folgesatz 1. Ist $R \in U$, so ist entweder $R^{\prime} \in O_{G}$ oder $R^{\prime} \in U . \quad$ Es ist $U \subset O_{A B}$.

Für die letzte Behauptung, die in [1] mittels der universellen Überlagerungsfläche bewiesen wurde, nehmen wir an, dass $f$ eine nichtkonstante beschränkte analytische Funktion auf $R$ ist. Wir bezeichnen mit $R^{\prime}$ eine Kreisscheibe, die die Werte von $f$ enthält. $\quad \mathrm{Da} R^{\prime}$ offenbar weder zu $U$ noch zu $O_{\theta}$ angehört, so haben wir einen Widerspruch erhalten.

Hilfssatz 2. Es sei u ein harmonisches Mass (bzw. eine singuläre Funktion)

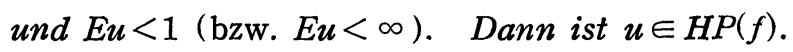

Es sei $G^{\prime}$ ein relativkompaktes Gebiet auf $R^{\prime}$. Dann ist

$$
\alpha=\sup \left\{u(p) \mid p \in f^{-1}\left(G^{\prime}\right)\right\} \leq \sup \left\{E u\left(p^{\prime}\right) \mid p^{\prime} \in G^{\prime}\right\}<1 \quad(\text { bzw. } \alpha<\infty)
$$

und

$$
f^{-1}\left(G^{\prime}\right) \subset\{p \in R \mid u<\alpha\}
$$

Dann ist nach (3)

$$
u \geq H_{f-1\left(G^{\prime}\right)}^{u} \geq H_{\{u<\alpha\}}^{u}=u,
$$

woraus $u \in H P(f)$ folgt. 
SATZ 8. Es sei $u \in H P(f)$ und Eu $+\infty$. Ist $u: a)$ quasibeschränkt $b$ ) singulär, c) minimal, d) diskret, e) ein harmonisches Mass, ${ }^{8)}$ so besitzt Eu dieselbe Eigenschaft. Ist $u=v+w$, wo $v$ quasibeschränkt und $w$ singulär ist, so ist

$$
E u=E v+E w
$$

a) Ist $u$ beschränkt, so ist auch $E u$ beschränkt. Ist $u$ quasibeschränkt, so gibt es eine nicht abnehmende Folge $\left\{u_{n}\right\}$ von beschränkten harmonischen Funktionen, die gegen $u$ streben. Aus dem Satz 4 folgt dann

$$
E u=\lim _{n \rightarrow \infty} E u_{n},
$$

woraus man gleich erkennt, dass $E u$ quasibeschränkt ist.

b) Es sei $u^{\prime}$ eine nichtnegative beschränkte harmonische Funktion auf $R^{\prime}$, für die $u^{\prime} \leq E u$ ist. Dann ist

$$
u \leq E u \circ f=u^{\prime} \circ f+\left(E u-u^{\prime}\right) \circ f .
$$

Mittels des Kjellbergschen Hilfssatzes kann man zwei harmonische Funktionen $u_{1}, u_{2}$ konstruieren, für welche $u=u_{1}+u_{2}, 0 \leq u_{1} \leq u^{\prime} \circ f, 0 \leq u_{2} \leq\left(E u-u^{\prime}\right) \circ f$ ist. Daraus folgt aber, dass $u_{1}$ beschränkt und, da $u$ singulär ist, sogar identisch Null ist. Es ist also

$$
u=u_{2} \leq\left(E u-u^{\prime}\right) \circ f,
$$

woraus man $E u \leq E u-u^{\prime}$ und $u^{\prime}=0$ folgert. Eu ist demnach singulär.

c) wurde im Satz 7 bewiesen.

d) Es sei $E u=u_{\tau}^{\prime}+u_{\sigma}^{\prime}$ die Zerlegung von $E u$ in eine total nicht diskrete Funktion $u_{\tau}^{\prime}$ und eine diskrete Funktion $u_{\sigma}^{\prime}$. Dann ist

$$
u \leq u_{\tau}^{\prime} \circ f+u_{\sigma}^{\prime} \circ f \text {. }
$$

$u_{\tau}^{\prime} \circ f$ ist total nichtdiskret. Denn aus $0<u_{0} \leq \boldsymbol{u}_{\tau}^{\prime} \circ f$, wo $\boldsymbol{u}_{0}$ eine minimale Funktion ist, folgt $0<E u_{0} \leq u_{\tau}^{\prime}$ und nach Satz 7 ist $E u_{0}$ minimal. Es ist daher $\boldsymbol{u} \leq \boldsymbol{u}_{\sigma}^{\prime} \circ \boldsymbol{f}, E \boldsymbol{u} \leq \boldsymbol{u}_{\sigma}^{\prime}$ und $\boldsymbol{u}_{\tau}^{\prime}=0$.

e) Aus $u \leq E u \circ f$ folgt

8) Heins hat bewiesen [7], dass wenn $u$ ein harmonisches Mass und Eu harmonisch ist, so ist auch $E u$ ein harmonisches Mass. Dieser Satz folgt aus dem Hilfssatz 2 und aus Satz 8. 


$$
\frac{u-\alpha}{1-\alpha} \leq \frac{E u-\alpha}{1-\alpha} \circ f \leq\left(\frac{E u-\alpha}{1-\alpha} \vee 0\right) \circ f
$$

für jedes $\alpha, 0<\alpha<1$, und nach Eigenschaft $(M)$ ist

$$
u=\frac{u-\alpha}{1-\alpha} \vee 0 \leq\left(\frac{E u-\alpha}{1-\alpha} \vee 0\right) \circ f
$$

Daraus erhalten wir

$$
E u \leq \frac{E u-\alpha}{1-\alpha} \vee 0
$$

Da $E u$ nicht grösser als 1 und nichtnegativ ist, so ist offenbar

$$
\frac{E u-\alpha}{1-\alpha} \leq E u \text {. }
$$

Daraus folgt, dass $E u$ die Eigenschaft $(M)$ besitzt und somit ein harmonisches Mass ist.

Es sei jetzt $u=v+w$, wo $v$ quasibeschränkt und $w$ singulär ist. Dann ist $v \wedge w=0$ und nach (2) ist $u=v \vee w$. Nach a) und b) sind $E v$ quasibeschränkt und $E w$ singulär und somit haben wir wie oben

$$
E v \vee E w=E v+E w .
$$

Nun ist nach Satz 1

$$
E u=E(v \vee w)=E v \vee E w=E v+E w,
$$

womit der Satz vollständig bewiesen ist.

\section{Der Operator $I_{f}$}

Definition. Es sei $u^{\prime} \in H P\left(R^{\prime}\right)$. Wir bezeichnen mit $I_{f} u^{\prime}$ die auf $R$ durch folgende Beziehung definierte Funktion:

$$
I_{f} u^{\prime}(p)=\sup \left\{u(p) \mid u \leq u^{\prime} \circ f, u \in H P(f)\right\} .
$$

Es sei $R_{\iota}$ eine Komponente von $R, R_{\iota}^{\prime}$ die Komponente von $R^{\prime}$ die $f\left(R_{\iota}\right)$ enthält und $f_{\iota}$ die Abbildung von $R_{\iota}$ in $R_{\iota}^{\prime}$ die mit $f$ zusammenfällt. Es sei $u^{\prime} \in H P\left(R^{\prime}\right)$ und $u_{\iota}^{\prime}$ die Einschränkung von $u^{\prime}$ auf $R_{\iota}^{\prime}$. Dann ist $I_{f_{\iota}} u_{\iota}^{\prime}=I_{f} u^{\prime}$ auf $R_{\iota}$.

Ist $R$ eine offene Menge auf $R^{\prime}$, und $f$ die identische Abbildung, so werden wir auch $I_{R}$ an Stelle von $I_{f}$ setzen, da $f$ implizite bekannt ist. Falls keine 
Missverständnisse $z \mathrm{u}$ befürchten sind, so werden wir auch einfach $I$ schreiben.

SATZ 9. I $u^{\prime}$ ist eine harmonische Funktion und $I u^{\prime} \in H P(f)$. Der Operator $I$ hat folgende Eigenschaften: $\left(u_{1}^{\prime}, u_{2}^{\prime} \in H P\left(R^{\prime}\right)\right)$

a) $I\left(u_{1}^{\prime} \vee u_{2}^{\prime}\right)=I u_{1}^{\prime} \vee I u_{2}^{\prime}, I\left(u_{1}^{\prime} \wedge u_{2}^{\prime}\right)=I u_{1}^{\prime} \wedge I u_{2}^{\prime}$,

b) $I\left(\alpha_{1} u_{1}^{\prime}+\alpha_{2} u_{2}^{\prime}\right)=\alpha_{1} I u_{1}^{\prime}+\alpha_{2} I u_{2}^{\prime}$,

wo $\alpha_{1}, \alpha_{2}$ reelle nichtnegative Zahlen sind.

Nach Eigenschaft c) von $H P(f)$ folgt, dass die Klasse $\left\{u \in H P(f) \mid u \leq u^{\prime} \circ f\right\}$ nach oben filtrant ist, woraus man erkennt, dass eine nichtabnehmende Folge aus dieser Klasse existiert, die gegen $I u^{\prime}$ konvergiert. Daraus und aus der Eigenschaft d) der Klasse $H P(f)$ folgt $I u^{\prime} \in H P(f)$.

Der Operator $I$ ist evident monoton und $I\left(\alpha u^{\prime}\right)=\alpha I u^{\prime} . \quad$ Aus $I u_{1}^{\prime}+I u_{2}^{\prime} \in H P(f)$ und

$$
I u_{1}^{\prime}+I u_{2}^{\prime} \leq u_{1}^{\prime} \circ f+u_{2}^{\prime} \circ f=\left(u_{1}^{\prime}+u_{2}^{\prime}\right) \circ f
$$

erhält man

$$
I u_{1}^{\prime}+I u_{2}^{\prime} \leq I\left(u_{1}^{\prime}+u_{2}^{\prime}\right)
$$

$\mathrm{Da}$

$$
I\left(u_{1}^{\prime}+u_{2}^{\prime}\right) \leq\left(u_{1}^{\prime}+u_{2}^{\prime}\right) \circ f=u_{1}^{\prime} \circ f+u_{2}^{\prime} \circ f
$$

ist, so kann man mittels des Hilfssatzes von Kjellberg zwei harmonische Funktionen $u_{1}, u_{2}$ konstruieren, derart dass

$$
I\left(u_{1}^{\prime}+u_{2}^{\prime}\right)=u_{1}+u_{2}, \quad 0 \leq u_{i} \leq u_{i}^{\prime} \circ \quad(i=1,2)
$$

ist. Aus $u_{i} \leq I\left(u_{1}^{\prime}+u_{2}^{\prime}\right)$ folgt sofort $u_{i} \in H P(f)$ und deshalb ist $u_{i} \leq I u_{i}^{\prime}$. Es ist somit

$$
I\left(u_{1}^{\prime}+u_{2}^{\prime}\right) \leq I u_{1}^{\prime}+I u_{2}^{\prime},
$$

woraus die Linearität von $I$ folgt.

Aus $u_{1}^{\prime} \wedge u_{2}^{\prime} \leq u_{i}^{\prime}(i=1,2)$, ergibt sich

$$
I\left(u_{1}^{\prime} \wedge u_{2}^{\prime}\right) \leq I u_{i}^{\prime}, I\left(u_{1}^{\prime} \wedge u_{2}^{\prime}\right) \leq I u_{1}^{\prime} \wedge I u_{2}^{\prime} .
$$

Da aber $I u_{1}^{\prime} \wedge I u_{2}^{\prime} \leq u_{i}^{\prime} \circ f$, so ist

$$
E\left(I u_{1}^{\prime} \wedge I u_{2}^{\prime}\right) \leq u_{i}^{\prime}
$$

und daraus folgt

$$
E\left(I u_{1}^{\prime} \wedge I u_{2}^{\prime}\right) \leq u_{1}^{\prime} \wedge u_{2}^{\prime}, \quad I u_{1}^{\prime} \wedge I u_{2}^{\prime} \leq\left(u_{1}^{\prime} \wedge u_{2}^{\prime}\right) \circ f,
$$




$$
I u_{1}^{\prime} \wedge I u_{2}^{\prime} \leq I\left(u_{1}^{\prime} \wedge u_{2}^{\prime}\right), \quad I u_{1}^{\prime} \wedge I u_{2}^{\prime}=I\left(u_{1}^{\prime} \wedge u_{2}^{\prime}\right)
$$

Daraus und aus

$$
\begin{aligned}
& \left(I u_{1}^{\prime} \vee I u_{2}^{\prime}\right)+\left(I u_{1}^{\prime} \vee I u_{2}^{\prime}\right)=I u_{1}^{\prime}+I u_{2}^{\prime}=I\left(u_{1}^{\prime}+u_{2}^{\prime}\right) \\
& \quad=I\left(\left(u_{1}^{\prime} \vee u_{2}^{\prime}\right)+\left(u_{1}^{\prime} \wedge u_{2}^{\prime}\right)\right)=I\left(u_{1}^{\prime} \vee u_{2}^{\prime}\right)+I\left(u_{1}^{\prime} \wedge u_{2}^{\prime}\right),
\end{aligned}
$$

ergibt sich

$$
I u_{1}^{\prime} \vee I u_{2}^{\prime}=I\left(u_{1}^{\prime} \vee u_{2}^{\prime}\right) .
$$

Es gelten folgende Rechenregeln: $\left(u \in H P(f), E u<\infty, u^{\prime} \in H P\left(R^{\prime}\right)\right)$

$$
\begin{array}{ll}
E I u^{\prime} \leq u^{\prime}, & I E I u^{\prime}=I u^{\prime}, \\
u \leq I E u, & E u=E I E u .
\end{array}
$$

Aus $I u^{\prime} \leq u^{\prime} \circ f$ folgt nähmlich

$$
E I u^{\prime} \leq u^{\prime}, \quad I u^{\prime} \leq\left(E I u^{\prime}\right) \circ f \leq u^{\prime} \circ f .
$$

Daraus folgt $I u^{\prime} \leq I E I u^{\prime} \leq I u^{\prime}$ und somit

$$
I u^{\prime}=I E I u^{\prime}
$$

Aus $u \leq E u^{\circ} f$ folgt

$$
u \leq I E u \leq E u \circ f, \quad E u \leq E I E u \leq E u
$$

und somit

$$
E I E u=E u
$$

Hilfssatz 3. Jede Funktion $u^{\prime} \in H P\left(R^{\prime}\right)$ ist in der Form $u^{\prime}=u_{1}^{\prime}+u_{2}^{\prime}$ darstellbar, wo $u_{1}^{\prime}$ das Bild durch $E$ einer Funktion aus $H P(f)$ ist und $u_{2}^{\prime}$ im Kern von I liegt. Diese Darstellung ist eindeutig. Ist $v^{\prime} \leq E u<\infty$ für ein $u \in H P(f)$, so ist

$$
v^{\prime}=E I v^{\prime}
$$

Es ist

$$
u_{1}^{\prime}=E I u^{\prime}, \quad u_{2}^{\prime}=u^{\prime}-E I u^{\prime}
$$

die gesuchte Zerlegung. Ist $u^{\prime}=u_{3}^{\prime}+u_{4}^{\prime}$ eine andere Darstellung von $u^{\prime}$ mit denselben Eigenschaften, so ist $E u=u_{3}^{\prime}$ für ein $u \in H P(f)$ und

$$
E I u_{3}^{\prime}=E I E u=E u=u_{3}^{\prime}
$$

Aber $I u^{\prime}=I u_{3}^{\prime}, \quad u_{1}^{\prime}=E I u^{\prime}=E I u_{3}^{\prime}=u_{3}^{\prime} \quad$ und $\quad u_{1}^{\prime}=u^{\prime}-u_{3}^{\prime}=u^{\prime}-u_{1}^{\prime}=u_{2}^{\prime}$. Aus $E u=v^{\prime}+\left(E u-v^{\prime}\right)$ folgt 


$$
\begin{gathered}
E u=E I E u \leq E I v^{\prime}+E I\left(E u-v^{\prime}\right) \leq E I v^{\prime}+E u-v^{\prime}, \\
v^{\prime} \leq E I v^{\prime}, \quad v^{\prime}=E I v^{\prime} .
\end{gathered}
$$

HilfsSATZ 4. Ist $G$ eine offene Menge auf $R$, derart dass jede Komponente von $G$ eine Greensche Funktion besitzt und ist $\eta$ die identische Abbildung von $G$ in $R$, so ist für jede Funktion $u \in H P(R)$

$$
u \circ \eta=I_{\eta} u+H_{G}^{u} \circ \eta
$$

Es ist offenbar $\left(u-H_{G}^{u}\right) \circ \eta \in \mathfrak{U}_{G}\left(\mathfrak{U}_{G}\right.$ wurde vor dem Satz 6 definiert $)$. Nach b), Satz 6 ist $\left(u-H_{G}^{u}\right) \circ \eta \in H P(\eta)$ und somit

$$
\left(u-H_{G}^{u}\right) \circ \eta \leq I_{\eta} u .
$$

$I_{r}, u$ gehört nach c), Satz 6 der Klasse $\mathfrak{H}_{\theta}$ an. Dann ist $u-\left(I_{r}, u\right) \circ \eta^{-1} \in \overline{\mathfrak{V}}_{\tilde{F}}^{\nu}$. Daraus folgt

$$
u-\left(I_{r} u\right) \circ \eta^{-1} \geq H_{G}^{u}, \quad u \circ \eta=I_{r} u+H_{G}^{u} \circ \eta .
$$

Laut des Hilfssatzes 4 ist, $u \in H P(f)$ gleichbedeutend $\operatorname{mit}_{f^{-1}\left(G^{\prime}\right)}^{I} u=0$ für jedes relativkompaktes Gebiet $G^{\prime}$ auf $R^{\prime}$.

SATz 10. Es sei $\left\{R_{n}^{\prime}\right\}$ eine nicht abnähmende Folge von offenen Mengen auf $R^{\prime}$, deren Vereinigung $R^{\prime}$ ist. Es sei $f_{n}$ die analytische Abbildung von $f^{-1}\left(R_{n}^{\prime}\right)$ in $R_{n}^{\prime}$, die mit $f$ zusammenfällt. Dann ist $I_{f_{n}} u^{\prime} \uparrow I_{f} u^{\prime}$ für jedes $u^{\prime} \in H P\left(R^{\prime}\right)$.

Ist $u \in H P\left(f_{n+1}\right)$, so ist $u$, als Funktion auf $f^{-1}\left(R_{n}^{\prime}\right)$ betrachtet, in der Klasse $H P\left(f_{n}\right)$ enthalten. Es sei nähmlich $G^{\prime}$ ein relativkompaktes Gebiet auf $R_{n}^{\prime}$; dann ist

$$
H_{f_{n}^{-1}\left(G^{\prime}\right)}^{u}=H_{f_{n+1}^{-1}\left(G^{\prime}\right)}^{u}=u
$$

auf $f_{n}^{-1}\left(G^{\prime}\right)$. Daraus folgt, dass die Folge $\left\{I_{f_{n}} u^{\prime}\right\}$ nicht zunähmend ist. Ähnlicherweise beweist man die Ungleichung $I_{f} u^{\prime} \leq I_{f_{n}} u^{\prime}$. Wir setzen

$$
v=\lim _{n \rightarrow \infty} I_{f_{n}} u^{\prime} \geq I_{f} u^{\prime}
$$

Es sei $G^{\prime}$ ein relativkompaktes Gebiet auf $R^{\prime}$. Für ein genügend grosses $n$ ist $\bar{G}^{\prime}$ in $R_{n}^{\prime}$ enthalten. Da $v \leq I_{f_{n}} u^{\prime} \in H P\left(f_{n}\right)$ ist, so folgt aus der Eigenschaft a) von $H P\left(f_{n}\right), v \in H P\left(f_{n}\right)$. Es ist also

$$
H_{f^{-1}\left(G^{\prime}\right)}^{v}=H_{f^{-1}\left(G^{\prime}\right)}^{v}=v
$$


auf $f^{-1}\left(G^{\prime}\right)$. Es ist also $v \in H P(f)$ und $v \leq I_{f} u^{\prime}$.

SATZ 11. Ist $u^{\prime}, u_{m}^{\prime} \in H P\left(R^{\prime}\right)(m=1,2, \ldots)$ und $u^{\prime} \geq \lim _{m \rightarrow \infty} u_{m}^{\prime}$, so ist

$$
I u^{\prime} \geq \varlimsup_{m \rightarrow \infty} I u_{m}^{\prime}
$$

Ist die Folge $\left\{u_{m}^{\prime}\right\}$ monoton und konvergent, so ist

$$
I \lim _{m \rightarrow \infty} u_{m}^{\prime}=\lim _{m \rightarrow \infty} I u_{m}^{\prime} \text {. }
$$

Es genügt den Satz im Falle dass $R^{\prime}$ zusammenhängend ist zu beweisen.

Wir werden erst beweisen, dass falls die Folge $\left\{u_{m}^{\prime} \circ f\right\}$ gleichmässig gegen $u^{\prime} \circ f$ konvergiert, so ist $I u^{\prime}=\lim _{m \rightarrow \infty} I u_{m}^{\prime}$. Für ein beliebiges $\varepsilon>0$ ist, für genügend grosse $m$,

$$
u^{\prime} \circ f \leq u_{m}^{\prime} \circ f+\varepsilon \leq u^{\prime} \cdot f+2 \varepsilon
$$

und deshalb auch

$$
I u^{\prime} \leq I u_{m}^{\prime}+I \varepsilon \leq I u^{\prime}+2 I \varepsilon,
$$

woraus die Behauptung folgt.

Wir nehmen jetzt an, dass $u^{\prime}=\lim _{m \rightarrow \infty} u_{m}^{\prime}$ ist, und es sei $\left\{R_{n}^{\prime}\right\}$ eine normale Ausschöpfung von $R^{\prime}$, und $f_{n}$ die analytische Abbildung von $f^{-1}\left(R_{n}^{\prime}\right)$ in $R_{n}^{\prime}$, die mit $f$ zusammenfällt. Auf $f^{-1}\left(R_{n}^{\prime}\right)$ konvergiert die Folge $\left\{u_{m}^{\prime} \circ f\right\}$ gleichmässig gegen $u^{\prime} \circ f$ und deshalb ist

$$
I_{f_{n}} u^{\prime}=\lim _{m \rightarrow \infty} I_{f_{n}} u_{m}^{\prime}
$$

Da aber nach dem Satz $10 I_{f_{n}} u_{m}^{\prime} \geq I_{f} u_{m}^{\prime}$ auf $f^{-1}\left(R_{n}\right)$ ist, so ist

$$
I_{f_{n}} u^{\prime} \geq \varlimsup_{m \rightarrow \infty} I_{f} u_{m}^{\prime} .
$$

Weiter ergibt sich aus demselben Satz

$$
I_{f} u^{\prime}=\lim _{n \rightarrow \infty} I_{f_{n}} u^{\prime} \geq \varlimsup_{m \rightarrow \infty} I_{f} u_{m}^{\prime} .
$$

Wir gehen jetzt zum allgemeineren Fall über, nähmlich dass $u^{\prime} \geq \varlimsup_{m \rightarrow \infty} u_{m}^{\prime}$ ist. Es sei $p \in R$ und $\left\{u_{m_{i}}^{\prime}\right\}$ eine konvergente Teilfolge von $\left\{u_{m}^{\prime}\right\}$, so dass die Folge $\left\{I u_{m_{i}}^{\prime}(p)\right\}$ konvergent und

$$
\lim _{i \rightarrow \infty} I u_{m_{i}}^{\prime}(p)=\varlimsup_{m \rightarrow \infty} I u_{m}^{\prime}(p)
$$

ist. Wir haben aus $u^{\prime} \geq \lim _{i \rightarrow \infty} u_{m_{\iota}}^{\prime}$, 


$$
I u^{\prime}(p) \geq\left(I \lim _{\imath \rightarrow \infty} u_{m_{i}}^{\prime}\right)(p) \geq \varlimsup_{i \rightarrow \infty} I u_{m_{i}}^{\prime}(p)=\varlimsup_{m \rightarrow \infty} I u_{m}^{\prime}(p),
$$

woraus die erste Behauptung des Satzes folgt.

Ist die Folge nicht zunehmend, so ist $I \lim _{n \rightarrow \infty} u_{n}^{\prime} \leq I u_{m}^{\prime}$ und deshalb

$$
I \lim _{n \rightarrow \infty} u_{n}^{\prime} \leq \lim _{m \rightarrow \infty} I u_{m}^{\prime}, \quad I \lim _{m \rightarrow \infty} u_{m}^{\prime}=\lim _{m \rightarrow \infty} I u_{m}^{\prime} .
$$

Ist die Folge nicht abnehmend, so ist $\lim _{n \rightarrow \infty} u_{n}^{\prime}-u_{m}^{\prime} \in H P\left(R^{\prime}\right)$ und

$$
I \lim _{n \rightarrow \infty} u_{n}^{\prime}=I u_{m}^{\prime}+I\left(\lim _{n \rightarrow \infty} u_{n}^{\prime}-u_{m}^{\prime}\right) .
$$

Da die Folge $\left\{\lim _{n \rightarrow \infty} u_{n}^{\prime}-u_{m}^{\prime}\right\}_{m}$ nicht zunehmend ist und gegen Null konvergiert, so ist

$$
I \lim _{m \rightarrow \infty} u_{m}^{\prime}=\lim _{m \rightarrow \infty} I u_{m}^{\prime}
$$

Hilfssatz 5. Es sei $u^{\prime} \in H P\left(R^{\prime}\right)$ und $G^{\prime}$ eine offene Menge auf $R^{\prime}$, für die jede Komponente eine Greensche Funktion besitzt. Ist $u^{\prime}=u_{R^{\prime}-G^{\prime}}^{\prime *}$, so ist

$$
I u^{\prime}=\left(I u^{\prime}\right)_{R-f^{-1}\left(G^{\prime}\right)}^{*}
$$

Wir können ohne Verlust der Allgemeinheit annehmen, dass $R^{\prime}$ zusammenhängend ist; es sei $\left\{R_{n}^{\prime}\right\}$ eine normale Ausschöpfung von $R^{\prime}, \eta_{n}$ die identische Abbildung von $R_{n}^{\prime} \cap G^{\prime}$ in $R_{n}^{\prime}$ und $\eta$ die identische Abbildung von $G^{\prime}$ in $R^{\prime}$. Aus dem Hilfssatz 4 folgt $I_{\eta_{n}} u^{\prime}=u^{\prime}$ auf $G^{\prime} \cap \partial R_{n}^{\prime}$ und $I_{\eta} u^{\prime}=0$. Nach dem Satz 10 ist also $\lim _{n \rightarrow \infty} I_{\eta_{n}} u^{\prime}=0$. Wir haben auf $f^{-1}\left(R_{n}^{\prime} \cap G^{\prime}\right)$

$$
I_{f} u^{\prime}=H_{f^{-1}\left(R^{\prime} n \cap G^{\prime}\right)}^{I_{f} u^{\prime}} \leq H_{f^{-1}\left(G^{\prime}\right)}^{I_{j} u^{\prime}}+\left(I_{\eta_{n}} u^{\prime}\right) \circ f .
$$

Für $n \rightarrow \infty$ erhalten wir

$$
I_{f} u^{\prime} \leq H_{f^{-1}\left(G^{\prime}\right)}^{I_{j} u^{\prime}}
$$

auf $f^{-1}\left(G^{\prime}\right)$, woraus folgt

$$
I_{f} u^{\prime}=\left(I_{f} u^{\prime}\right)_{R-f^{-1}\left(G^{\prime}\right)}^{*}
$$

SATZ 12. Es sei $R \stackrel{f}{\longrightarrow} R^{\prime} \stackrel{f^{\prime}}{\longrightarrow} R^{\prime \prime}$. Dann ist

$$
I_{f^{\prime} \circ f}=I_{f} I_{f^{\prime}} .
$$

Wir bezeichnen $f^{\prime \prime}=f^{\prime} \circ f$. Es sei $u^{\prime \prime} \in H P\left(R^{\prime \prime}\right), G^{\prime \prime}$ ein relativkompaktes Gebiet auf $R^{\prime \prime}$ und $G^{\prime}=f^{\prime-1}\left(G^{\prime \prime}\right)$. Da 


$$
I_{f^{\prime}} u^{\prime \prime}=\left(I_{f}, u^{\prime \prime}\right)_{R^{\prime}-G^{\prime}}^{*}
$$

ist, so ist nach dem vorangehender Hilfssatz

$$
I_{f} I_{f}, u^{\prime \prime}=\left(I_{f} I_{f}, u^{\prime \prime}\right)_{R-f^{\prime \prime-1}\left(G^{\prime \prime}\right)}^{*},
$$

d.h. $I_{f} I_{f}, u^{\prime \prime} \in H P\left(f^{\prime \prime}\right)$. Daraus und aus $I_{f} I_{f}, u^{\prime \prime} \leq u^{\prime \prime \circ} f$ folgt

$$
I_{f} I_{f^{\prime}} u^{\prime \prime} \leq I_{f^{\prime \prime}} u^{\prime \prime} \text {. }
$$

Aus $u \in H P\left(f^{\prime \prime}\right)$ und $E_{f} u<\infty$ folgt $E_{f} u \in H P\left(f^{\prime}\right)$. Denn es sei $G^{\prime \prime}$ ein relativkompaktes Gebiet auf $R^{\prime \prime}$; dann ist

$$
u=u_{R-f^{\prime \prime-1}\left(G^{\prime \prime}\right)}^{*}=u_{R-f^{-1}\left(f^{\prime-1}\left(f^{\prime \prime}\right)\right)}^{*}
$$

und aus Hilfssatz 1 haben wir

$$
E_{f} u=\left(E_{f} u\right)_{R^{\prime}-f^{\prime-1}\left(G^{\prime \prime}\right)}^{*} .
$$

Daraus erkennt man erst, dass $E_{f} u$ harmonisch auf $R^{\prime}$ ist, denn jeder Punkt von $R^{\prime}$ kann innerer Punkt einer Menge der Form $f^{\prime-1}\left(G^{\prime \prime}\right)$ werden. Weiter folgt aus der letzten Gleichheit $E_{f} u \in H P\left(f^{\prime}\right)$.

Es ist $H P\left(f^{\prime \prime}\right) \subset H P(f)$. In der Tat, es sei $u \in H P\left(f^{\prime \prime}\right)$ und $G^{\prime}$ ein relativkompaktes Gebiet auf $R^{\prime}$. Dann ist $f^{\prime}\left(G^{\prime}\right)$ ein relativkompaktes Gebiet auf $R^{\prime \prime}$ und $f^{-1}\left(G^{\prime}\right) \subset f^{\prime \prime-1}\left(f^{\prime}\left(G^{\prime}\right)\right)$. Daraus folgt

$$
u=u_{R-f^{\prime \prime-1}\left(f^{\prime}\left(G^{\prime}\right)\right)}^{*} \leq u_{R-f^{-1}\left(G^{\prime}\right)}^{*} \leq u
$$

und $u \in H P(f)$.

Aus $I_{f^{\prime \prime}} u^{\prime \prime} \in H P\left(f^{\prime \prime}\right)$ folgt $E_{f} I_{f^{\prime \prime}} u^{\prime \prime} \in H P(f)$. Es ist aber

$$
I_{f^{\prime \prime}} u^{\prime \prime} \leq u^{\prime \prime} \circ f^{\prime \prime}=\left(u^{\prime \prime} \circ f^{\prime}\right) \circ f
$$

und deshalb ist

$$
\begin{gathered}
E_{f} I_{f^{\prime \prime}} u^{\prime \prime} \leq u^{\prime \prime \circ} \circ, \quad E_{f} I_{f^{\prime \prime}} u^{\prime \prime} \leq I_{f^{\prime}} u^{\prime \prime}, \\
I_{f^{\prime \prime}} u^{\prime \prime} \leq\left(E_{f} I_{f^{\prime \prime}} u^{\prime \prime}\right) \circ f \leq\left(I_{f}, u^{\prime \prime}\right) \circ f .
\end{gathered}
$$

Daraus und aus $I_{f^{\prime \prime}} u^{\prime \prime} \in H P(f)$ ergibt sich

$$
I_{f^{\prime \prime}} u^{\prime \prime} \leq I_{f} I_{f}, u^{\prime \prime}, \quad I_{f \prime \prime} u^{\prime \prime}=I_{f} I_{f}, u^{\prime \prime} .
$$

Hilfssatz 6. Es sei $R^{\prime}$ zusammenhängend, $G^{\prime}$ eine offene Menge auf $R^{\prime}$ und $G=f^{-1}\left(G^{\prime}\right)$. Wir bezeichnen mit $f^{\prime}$ die Abbildung von $G$ in $G^{\prime}$ die mit $f$ zusammenfällt. Es sei $u^{\prime}$ eine minimale Funktion auf $R^{\prime}$ für die $\underset{G^{\prime}}{I} u^{\prime} \neq 0, I_{f} u^{\prime} \neq 0$ ist. Dann ist $G \neq \phi$ und 


$$
\underset{G}{I} I_{f} u^{\prime}=I_{f^{\prime}} I_{G^{\prime}} u^{\prime} \neq 0, \quad \underset{G G}{E I} I_{f} u^{\prime}=I_{f} u^{\prime} .
$$

Es sei erstens $G \neq \phi$. Wir setzen $u=I_{f} u^{\prime}$. Ist $I_{G} u=0$, so ist, nach Hilfssatz 4, $u=H_{G}^{u}$ auf $G$. Da $\overline{\mathfrak{V}}_{G^{\prime}}^{u^{\prime} \circ} f \subset \overline{\mathfrak{V}}_{G}^{u}$ ist, so ist $H_{G}^{u} \leq H_{G^{\prime}}^{u^{\prime} \circ} f$ und sommit $u \leq H_{G^{\prime}}^{u^{\prime} \circ} f$ auf $G$ und

$$
u \leq u_{R^{\prime}-G^{\prime}}^{*} \circ f .
$$

Ist $G=\phi$, so ist offenbar $u \leq u_{R^{\prime}-G^{\prime}}^{*} \circ f$. Es ist also in beiden Fällen ( $G=\phi$, oder $G \neq \phi$ und $\left.I_{G} I_{f} u^{\prime}=0\right)$. $\quad E_{f} u \leq u_{R^{\prime}-G^{\prime}}^{\prime *} \leq u^{\prime}$. Da aber $u^{\prime}$ minimal ist, so ist

$$
\begin{gathered}
E_{f} I_{f} u^{\prime}=\alpha u^{\prime}, \quad I_{f} u^{\prime}=I_{f} E_{f} I_{f} u^{\prime}=\alpha I_{f} u^{\prime}, \\
\alpha=1, u^{\prime}=u_{R^{\prime}-G^{\prime}}^{\prime *} .
\end{gathered}
$$

Aus dem Hilfssatz 4 folgt dann $\underset{G^{\prime}}{I} u^{\prime}=0$ entgegen der Voraussetzung. Es ist also $G \neq \phi$ und $I_{G} I_{f} u^{\prime} \neq 0$. Die Gleichheit

$$
\underset{G}{I} I_{f} u^{\prime}=I_{f^{\prime}} \underset{G^{\prime}}{I} u^{\prime}
$$

folgt aus dem Satz 12. Wir bezeichnen $u_{0}=u-\underset{G G}{E I} u$. Es ist

$$
I_{G} u_{0}=0, \quad u_{0}=H_{G}^{u_{0}} .
$$

Aus $u_{0} \leq u \leq u^{\prime} \circ f$ folgt $u_{0} \in H P(f)$ und

$$
E_{f} u_{0} \leq u^{\prime}, \quad E_{f} u_{0}=\alpha u^{\prime} \quad(0 \leq \alpha \leq 1) .
$$

Es ist also

$$
u_{0} \leq I_{f} E_{f} u_{0}=\alpha u=\alpha u_{0}+\alpha \underset{G G}{E I} u .
$$

Es sei $\alpha=1$. Aus $u_{0}=H_{G}^{u_{0}} \leq H_{G^{\prime}}^{u} \leq H_{G^{\prime}}^{u^{\prime} \circ f}$ folgt

$$
u_{0} \leq u_{R^{\prime}-G^{\prime}}^{\prime *} \circ, \quad u^{\prime}=E_{f} u_{0} \leq u_{R^{\prime}-G^{\prime}}^{\prime *} \leq u^{\prime} .
$$

Daraus folgert man $\underset{G}{I} u^{\prime}=0$ entgegen der Voraussetzung. Es ist also $\alpha<1$. Aus $(1-\alpha) u_{0} \leq \alpha \underset{G G}{E I} u$ und Hilfssatz 3 folgt

$$
u_{0}=\underset{G G}{E I} u_{0}=0, \quad u=\underset{G G}{E I} u,
$$

was $\mathrm{zu}$ beweisen war.

Folgesatz 2. Sind $G_{1}, G_{2}$ zwei offene Mengen und $u$ eine minimale Funktion auf $R(R$ zusammenhängend $)$ für die $\underset{G_{i}}{I} u \neq 0 \quad(i=1,2)$ ist, so ist $G_{1} \cap G_{2} \neq \phi$ und

$$
\underset{G_{1} \cap G_{2}}{I} u \neq 0 .
$$


Ist $I_{G} u \neq 0$ für eine offene Menge $G$, so gibt es nur eine Komponente $G_{0}$ von $G$. derart dass

$$
\underset{G_{0}}{I} u \neq 0
$$

ist.

Dieser Folgesatz folgt unmittelbar aus dem Hilfssatz 6 , wenn man $R, G_{1}$, $G_{2}, G_{1} \cap G_{2}$ an Stelle von $R^{\prime}, R, G^{\prime}, G$ setzt.

SATz 13. Es seien $R$ und $R^{\prime}$ zusammenhängend und $u^{\prime} \in H P\left(R^{\prime}\right)$. Ist $u^{\prime}$ a) singulär, b) quasibeschränkt, c) total nicht diskret, d) ein harmonisches Mass, so besitzt I $u^{\prime}$ dieselben Eigenschaften. Die Funktion $u^{\prime} \circ f-I u^{\prime}$ ist quasibeschränkt.

a) und b). Es sei $u \in H P(R)$,

$$
u \leq I u^{\prime}
$$

Da $I u^{\prime}$ der Klasse $H P(f)$ angehört, so gehört auch $u$ der Klasse $H P(f)$ an und nach dem Satz 5 ist $E u$ harmonisch. Aus obiger Ungleichung folgt noch $E u \leq u^{\prime}$.

Ist $u^{\prime}$ singulär und $u$ beschränkt, so ist auch $E u$ beschränkt und somit Null. Es ist also $u$ Null und $I u^{\prime}$ singulär. Ist $u^{\prime}$ quasibeschränkt und $u$ singulär, so ist auch $E u$ singulär und somit Null. Es ist also $u$ Null und $I u^{\prime}$ quasibeschränkt.

c) Ist $u \leq I u^{\prime}$ und $u$ minimal, so ist $E u \leq E I u^{\prime} \leq u^{\prime}$ und $E u$ ist nach Satz 8 harmonisch und minimal. Da $u^{\prime}$ total nichtdiskret ist, so sind $E u$ und $u$ Null und $I u^{\prime}$ ist total nichtdiskret.

d) Es ist

$$
\left(1-I u^{\prime}\right) \wedge I u^{\prime}+I u^{\prime} \leq\left(1-I u^{\prime}\right)+I u^{\prime}=1 .
$$

Da aber $\left(1-I u^{\prime}\right) \wedge I u^{\prime}$ und $I u^{\prime}$ der Klasse $H P(f)$ angehören, so ist

$$
\left(1-I u^{\prime}\right) \wedge I u^{\prime}+I u^{\prime} \leq I 1
$$

und

$$
\left(1-I u^{\prime}\right) \wedge I u^{\prime} \leq I\left(1-u^{\prime}\right)
$$

Daraus folgt

$$
\left(1-I u^{\prime}\right) \wedge I u^{\prime} \leq I\left(1-u^{\prime}\right) \wedge I u^{\prime}=I\left(\left(1-u^{\prime}\right) \wedge u^{\prime}\right)=0
$$

und $I u^{\prime}$ ist ein harmonisches Mass. 
Es sei $u$ singulär $u \leq u^{\prime} \circ f-I u^{\prime}$. Dann ist $E u \leq u^{\prime}<\infty$ und nach Hilfssatz 2 ist $u \in H P(f)$. Aus der Eigenschaft $\mathrm{b})$ der Klasse $H P(f)$ folgt $u+I u^{\prime} \in$ $H P(f)$ und somit

$$
u+I u^{\prime} \leq I u^{\prime}, \quad u=0 .
$$

Aus $u^{\prime}$ minimal folgt nicht immer $I u^{\prime}$ minimal.

\section{Der ideale Rand von Martin}

Es sei $R$ eine (zusammenhängende) Riemannsche Fläche mit Greenscher Funktion, $g_{q}$ die Greensche Funktion von $R$ mit dem Pol in $q$,

$$
K_{q}(p)=\frac{g_{q}(p)}{g_{q}\left(p_{0}\right)} \quad\left(q \neq p_{0}\right)
$$

die Funktion von Martin ( $p_{0}$ ist hier ein fixierter Punkt auf $R$ ), $\Delta$ der ideale Rand von $R$ im Sinne von Martin und $\Delta_{1}$ der Teil der Minimalen aus $\Delta$ [11], [13]. $\hat{R}=R \cup \Delta$ ist ein metrischer kompakter Raum. Für zwei Punkte $\hat{p}$, $\hat{q} \in \hat{R}$ bezeichnen wir mit $d(\hat{p}, \hat{q})$ ihre Entfernung in der Metrik von Martin. Martin hat bewiesen, dass jede nichtnegative harmonische Funktion $u$ auf $R$, $u \in H P(R)$, mittels einer Integrale der Form

$$
u=\int_{\Delta} K_{s} d \mu(s)
$$

dargestellt werden kann, wobei $\mu$ ein Borelsches Mass ist. Falls $\mu\left(\Delta-\Delta_{1}\right)=0$ ist, so nennt man $\mu$ ein kanonisches Mass; jede Funktion $u \in H P(R)$ ist mittels eines kanonischen Masses in dieser Form eindeutig darstellbar.

Es sei $S \in S P(R)$ und $F$ eine abgeschlossene Menge in $R$. Die superharmonische Funktion $S_{F}^{*}$ ist eindeutig in der Form

$$
S_{F}^{*}=\int_{\bar{F} \cap\left(R \cup \Delta_{1}\right)} K_{q} d \mu(q)+g_{p_{0}} \mu\left(\left\{p_{0}\right\}\right)
$$

darstellbar, wo $\bar{F}$ die abgeschlossene Hülle von $F$ in $\hat{R}$ und $\mu$ ein Borelsches Mass ist. Für $F=R$ erhält man

$$
S=\int_{R \cup \Delta_{1}} K_{q} d \mu(q)+g_{p_{0}} \mu\left(\left\{p_{0}\right\}\right)=\int_{R} K_{q} d \mu(q)+g_{p_{0} \mu}\left(\left\{p_{0}\right\}\right)+\int_{\Delta_{1}} K_{s} d \mu(s) .
$$

Die erste Integrale ist ein Potential und die zweite eine harmonische Funktion, also ist diese Zerlegung gerade der Satz von Riesz. 
Es sei $A$ eine abgeschlossene Menge auf $\Delta, A_{n}$ die Menge der Punkte von $R$, deren Entfernung von $A$ nicht grösser als $\frac{1}{n}$ ist und

$$
u=\int_{\Delta_{1}} K_{s} d \mu(s) \in H P(R) .
$$

Es ist, wie Martin bewiesen hat [11],

$$
\lim _{n \rightarrow \infty} u_{A_{n}}^{*}=\int_{A} K_{S} d \mu(s) .
$$

Es sei $s \in \Delta_{1}$ und

$$
G_{\alpha}=\left\{p \in R \mid K_{s}(p)>\alpha\right\} \quad\left(0<\alpha<\sup K_{s}\right) .
$$

Dann ist $K_{s}-\alpha \in \mathfrak{H}_{\sigma_{\alpha}}$ und aus b) Satz 6 folgt $I_{\sigma_{\alpha}} K_{s} \geq K_{s}-\alpha \neq 0$.

Es sei $s \in \Delta_{1}, \hat{G}$ eine Umgebung von $s$ auf $\hat{R}$ und $G=\hat{G} \cap R$. Dann ist

$$
\left(K_{S}\right)_{R-G}^{*}=\int_{(\overline{R-G}) \cap\left(R \cup \Delta_{1}\right)} K_{q} d \mu(q) .
$$

Ist $\underset{G}{I} K_{s}=0$, so ist nach Hilfssatz 4

$$
H_{G}^{K_{s}}=K_{s}, \quad \int_{(\overline{R-G}) \cap\left(R \cup \Delta_{1}\right)} K_{q} d \mu(q)=\left(K_{s}\right)_{R-G}^{*}=K_{s}
$$

entgegen der Eindeutigkeit solcher Darstellungen. Es ist somit [10]

$$
I_{G} K_{s} \neq 0 .
$$

Jeder Punkt $s \in \Delta_{1}$ ist ein erreichbarer Randpunkt von $R$. Es sei $G(s, \varepsilon)$ die Menge der Punkte von $R$ deren Entfernung (in der Metrik von Martin) von $s$ kleiner als $\varepsilon$ ist. Laut obiger Bemerkung ist $\underset{G(s, \varepsilon)}{I} K_{s} \neq 0$; es sei $G_{n}$ diejenige Komponente (Folgesatz 2) von $G\left(s, \frac{1}{n}\right)$, für die $\underset{G_{n}}{I} K_{s} \neq 0$ ist. Nach Folgesatz 2 ist $G_{n} \cap G_{n+1} \neq \phi$ und somit $G_{n+1} \subset G_{n}$. Man kann also einen Weg auf $R$ konstruieren, der gegen $s$ strebt.

HILFSSATZ a. Es seien $u_{1}, u_{2} \in H P(R)$ und

$$
u_{i}=\int_{\Delta_{1}} K_{s} d \mu_{i}(s) \quad(i=1,2)
$$

Ist $u_{1} \leq u_{2}$, so gibt es eine messbare Funktion $\theta$ auf $\Delta_{1}, 0 \leq \theta \leq 1$, so dass für jede Borelsche Menge $A$ 


$$
\mu_{1}(A)=\int_{A} \theta(s) d \mu_{2}(s)
$$

ist. Ist

$$
u_{i}=\int_{\Delta_{1}} K_{s} \theta_{i}(s) d \mu(s)
$$

wo $\theta_{i}$ eine nichtnegative in Bezug auf $\mu$ sumierbare Borelsche Funktion ist, so ist auch

$$
\theta_{1} \leq \theta_{2}:[\mu]^{9)}
$$

Es sei $u_{0}=u_{2}-u_{1}=\int_{\Delta_{1}} K_{s} d \mu_{0}(s)$. Aus

$$
\int_{\Delta_{1}} K_{s} d \mu_{2}(s)=u_{2}=u_{1}+u_{0}=\int_{\Delta_{1}} K_{s} d\left(\mu_{1}+\mu_{0}\right)(s)
$$

und aud der Eindeutigkeit der kanonischen Darstellung folgt

$$
\mu_{2}=\mu_{1}+\mu_{0} \geq \mu_{1}{ }^{10)}
$$

Daraus erkennt man, dass $\mu_{1}$ absolut stetig ([3], Seite 132) in Bezug auf $\mu_{2}$ ist und der Hilfssatz folgt aus dem Satz von Radon-Nykodim.

Es seien $\sigma$ eine endliche totaladditive Mengenfunktion, auf der Klasse der Borelschen Mengen von $\Delta_{1}$. Der Satz von Hahn ([3], Seite 121) behauptet, dass man eine Zerlegung von $\Delta_{1}$ in zwei Borelsche Mengen $A^{+}, A^{-}$finden kann,

$$
A^{+} \cup A^{-}=\Delta_{1}, \quad A^{+} \cap A^{-}=\phi,
$$

derart dass für $A \subset A^{+}$(bzw. $A \subset A^{-}$)

$$
\sigma(A) \geq 0, \quad(\text { bzw. } \sigma(A) \leq 0)
$$

ist. Man nennt $A^{+}$eine positive Menge und $A^{-}$eine negative Menge für $\sigma$. Man bezeichnet mit $\sigma^{+}, \sigma^{-},|\sigma|$ die Borelschen Masse,

$$
\begin{gathered}
\sigma^{+}(A)=\sigma\left(A \cap A^{+}\right), \quad \sigma^{-}(A)=-\sigma\left(A \cap A^{-}\right), \\
|\sigma|(A)=\sigma^{+}(A)+\sigma^{-}(A)
\end{gathered}
$$

und man nennt sie die positive, bzw. negative, bzw. totale Variation von $\sigma$. Für $A \subset A^{+}$ist

$$
|\sigma|(A)=\sigma^{+}(A)=\sigma(A)
$$

9) D.h. bis auf eine Menge von $\mu$-Masse Null.

10) D.h. $\mu_{2}(A) \geq \mu_{1}(A)$ für jede Borelsche Menge $A$. 
und für $A \subset A^{-}$

$$
|\sigma|(A)=\sigma^{-}(A)=-\sigma(A) .
$$

Es seien $\sigma_{1}, \sigma_{2}$ zwei endliche totaladditive Mengenfunktionen. Wir setzen

$$
\begin{aligned}
& \sigma_{1} \vee \sigma_{2}=\frac{1}{2}\left(\sigma_{1}+\sigma_{2}+\left|\sigma_{1}-\sigma_{2}\right|\right), \\
& \sigma_{1} \wedge \sigma_{2}=\frac{1}{2}\left(\sigma_{1}+\sigma_{2}-\left|\sigma_{1}-\sigma_{2}\right|\right) .
\end{aligned}
$$

Diese endlichen totaladditiven Mengenfunktionen besitzen folgende Eigenschaft : sind $\bar{\sigma}, \underline{\sigma}$ zwei endliche totaladditive Mengenfunktionen, für die $\underline{\sigma} \leq \sigma_{i} \leq \bar{\sigma}$ $(i=1,2)$ ist, so ist

$$
\underline{\sigma} \leq \sigma_{1} \wedge \sigma_{2} \leq \sigma_{i} \leq \sigma_{1} \vee \sigma_{2} \leq \bar{\sigma} .
$$

Es sei nähmlich $A^{+}$(bzw. $A^{-}$) eine positive (bzw. negative) Menge für $\sigma_{1}-\sigma_{2}$. Es ist für eine Borelsche Menge $A$

$$
\begin{aligned}
\sigma_{1} & \wedge \sigma_{2}(A)=\sigma_{1} \wedge \sigma_{2}\left(A \cap A^{+}\right)+\sigma_{1} \wedge \sigma_{2}\left(A \cap A^{-}\right)= \\
& =\frac{1}{2}\left(\sigma_{1}\left(A \cap A^{+}\right)+\sigma_{2}\left(A \cap A^{+}\right)-\left(\sigma_{1}-\sigma_{2}\right)\left(A \cap A^{+}\right)+\sigma_{1}\left(A \cap A^{-}\right)\right. \\
& +\sigma_{2}\left(A \cap A^{-}\right)+\left(\sigma_{1}-\sigma_{2}\right)\left(A \cap A^{-}\right)=\sigma_{2}\left(A \cap A^{+}\right)+\sigma_{1}\left(A \cap A^{-}\right) .
\end{aligned}
$$

Daraus folgt

$$
\begin{aligned}
& \underline{\sigma}(A)=\underline{\sigma}\left(A \cap A^{+}\right)+\underline{\sigma}\left(A \cap A^{-}\right) \leq \sigma_{2}\left(A \cap A^{+}\right)+\sigma_{1}\left(A \cap A^{-}\right) \\
& \quad=\sigma_{1} \wedge \sigma_{2}(A) \leq \sigma_{i}(A)
\end{aligned}
$$

Ähnlicherweise beweist man die entsprechende Eigenschaft von $\sigma_{1} \vee \sigma_{2}$ Insbesondere ist

$$
\sigma \vee 0=\sigma^{+}, \quad \sigma \wedge 0=-\sigma^{-} .
$$

Hilfssatz b. Es seien $u_{1}, u_{2}$ Differenzen von Funktionen aus $H P(R) . u_{i}$ gestattet eine Darstellung der Form

$$
u_{i}=\int_{\Delta_{1}} K_{s} d \sigma_{i}(s)
$$

wo $\sigma_{i}$ eine endliche totaladditive Mengenfunktion ist. Dann ist

$$
\begin{aligned}
& u_{1} \vee u_{2}=\int_{\Delta_{1}} K_{s} d\left(\sigma_{1} \vee \sigma_{2}\right)(s), \\
& u_{1} \wedge u_{2}=\int_{\Delta_{1}} K_{s} d\left(\sigma_{1} \wedge \sigma_{2}\right)
\end{aligned}
$$


Ist

$$
u_{i}=\int_{\Delta_{1}} K_{s} \theta_{i}(s) d \mu(s),
$$

wo $\mu$ ein (nichtnegatives) Mass ist, so ist

$$
\begin{aligned}
& u_{1} \vee u_{2}=\int_{\Delta_{1}} K_{s} \max \left(\theta_{1}(s), \theta_{2}(s)\right) d \mu(s), \\
& u_{1} \wedge u_{2}=\int_{\Delta_{1}} K_{s} \min \left(\theta_{1}(s), \theta_{2}(s)\right) d \mu(s) .
\end{aligned}
$$

Wir setzen $\mu_{i}=\left(\sigma_{1} \vee \sigma_{2}\right)-\sigma_{i}(i=1,2)$. Da $\mu_{i}$ ein Mass ist, so ist

$$
v_{i}=\int_{\Delta_{1}} K_{s} d \mu_{i}(s)
$$

nichtnegativ. Aus

$$
u_{i}+v_{i}=\int_{\Delta_{1}} K_{s} d\left(\sigma_{1} \vee \sigma_{2}\right)(s)
$$

folgt

$$
\begin{gathered}
u_{i} \leq \int_{\Delta_{1}} K_{s} d\left(\sigma_{1} \vee \sigma_{2}\right)(s), \\
u_{1} \vee u_{2} \leq \int_{\Delta_{1}} K_{s} d\left(\sigma_{1} \vee \sigma_{2}\right)(s) .
\end{gathered}
$$

Wir setzen $u_{1} \vee u_{2}=\int_{\Delta_{1}} K_{s} d \bar{\sigma}(s) . \quad \mathrm{Da}$

$$
u_{1} \vee u_{2}-u_{i}=\int_{\Delta_{1}} K_{s} d\left(\bar{\sigma}-\sigma_{i}\right)(s)
$$

nichtnegativ ist, so ist $\bar{\sigma}-\sigma_{i}$ ein Mass und somit

$$
\begin{aligned}
\sigma_{i} & \leq \bar{\sigma}, \quad \sigma_{1} \vee \sigma_{2} \leq \bar{\sigma} \\
\int_{\Delta_{1}} K_{s} d\left(\sigma_{1} \vee \sigma_{2}\right) \quad(s) & \leq \int_{\Delta_{1}} K_{s} d \bar{\sigma}(s)=u_{1} \vee u_{2}
\end{aligned}
$$

Ähnlicherweise verläuft der Beweis für $u_{1} \wedge u_{2}$. Setzen wir

$$
\sigma_{i}(A)=\int_{A} \theta_{i}(s) d \mu(s)
$$

so ist

$$
\sigma_{1} \vee \sigma_{2}(A)=\int_{A} \max \left(\theta_{1}(s), \theta_{2}(s)\right) d \mu(s),
$$

woraus die letzte Behauptung des Hilfssatzes folgt.

Wir werden mit $\chi_{R}$ das kanonische Mass der Funktion $1 \in \operatorname{HP}(R)$ 
bezeichnen :

$$
1=\int_{\Delta_{1}} K_{s} d \chi_{R}(s)
$$

wir werden einfach $\chi$ schreiben, wenn $R$ aus dem Text ersichltlich ist. Es sei $s \in \Delta ; \quad \chi(\{s\}) \neq 0$ ist gleichbedeutend mit $s \in \Delta_{1}$, und $K_{s}$ beschränkt. Das harmonische Mass der Borelschen Menge $A \subset \Delta$ soll die Funktion

$$
\omega(A)=\omega(A, R)=\int_{A} K_{s} d \chi_{R}(s)
$$

sein [13]. Es ist nach Hilfssatz b

$$
\begin{gathered}
\omega(A) \vee \omega(B)=\omega(A \cup B), \quad \omega(A) \wedge \omega(B)=\omega(A \cap B), \\
\omega(A) \wedge(1-\omega(A))=0 .
\end{gathered}
$$

Das harmonische Mass einer Borelschen Menge ist somit ein harmonisches Mass (Siehe Seite 13). Umgekehrt, ist $u$ ein harmonisches Mass, so gibt es eine Borelsche Menge $A \subset \Delta_{1}$ derart, dass $u=\omega(A)$ ist. In der Tat, nach Hilfssatz a ist

$$
u=\int_{\Delta_{1}} K_{s} \theta(s) d \chi(s)
$$

$0 \leq \theta \leq 1$. Daraus folgt

$$
\begin{aligned}
& 0=u \wedge(1-u)=\int_{\Delta_{1}} K_{s} \min (\theta(s), 1-\theta(s)) d \chi(s), \\
& \min (\theta(s), 1-\theta(s))=0:[\chi] .
\end{aligned}
$$

Wir setzen $A=\left\{s \in \Delta_{1} \mid \theta(s)=1\right\}, B=\left\{s \in \Delta_{1} \mid \theta(s)=0\right\}$. Obige Gleichheit liefert

$$
A \cup B=\Delta_{1}:[\chi]
$$

und das gibt $u=\omega(A)$.

Eine (nicht unbedingt Borelsche) Menge $A \subset \Delta$ heisst vom harmonischen Masse Null, wenn

$$
\inf \{\chi(\Gamma) \mid A \subset \Gamma \subset \Delta, \Gamma \text { offen }\}=0
$$

ist. $\Delta-\Delta_{1}$ ist vom harmonischen Masse Null. Wir werden den Ausdruck fast überall auf $A$ für eine Eigenschaft benutzen, wenn alle Punkte aus $A$ diese Eigenschaft besitzen, bis auf eine Menge vom harmonischen Masse Null.

Es sei $\sigma$ eine endliche totaladditive Mengenfunktion auf $\Delta_{1}$. Nach dem Satz von Lebesgue ([3], Seite 134) kann man $\sigma$ eindeutig in zwei endliche 
Megenfunktionen zerlegen,

$$
\sigma=\sigma_{0}+\sigma_{1}
$$

wobei $\sigma_{1}$ absolut stetig in Bezug auf $\%$ und $\sigma_{\text {s }}$ orthogonal $z u$ ist; d.h. man kann eine Zerlegung von $\Delta_{1}$ in zwei Borelschen Mengen finden,

$$
\Gamma_{0} \cup \Gamma_{1}=\Delta_{1}, \quad \Gamma_{0} \cap \Gamma_{1}=\phi,
$$

so dass

$$
\%\left(\Gamma_{0}\right)=0, \quad\left|\sigma_{0}\right|\left(\Gamma_{1}\right)=0
$$

ist. Es sei $u$ eine Differenz von $z$ wei Funktionen aus $H P(R)$ :

$$
u=\int_{\Delta_{1}} K_{s} d_{\sigma}(s)
$$

Zerlegt man $\sigma$, wie oben gezeigt wurde, so ist $u$ in der Form

$$
u=\int_{\Delta_{1}} K_{s} \theta(s) d \%(s)+\int_{\Delta_{1}} K_{s} d \sigma_{0}(s)
$$

darstellbar, wo die erste Funktion eine Differenz von zwei quasibeschränkten und die zweite eine Differenz von zwei singulären Funktionen sind. Das ist gerade die von Parreau angegebene Zerlegung einer Funktion aus $H P(R)$ in einer quasibeschränkten und einer singulären Funktion.

Es sei $u \in H P(R)$ :

$$
u=\int_{\Delta_{1}} K_{s} d \mu(s)
$$

es gibt höchstens abzählbar viele $s \in \Delta_{1}$, die positives $\mu$-Mass haben. Für jede Borelsche Menge $A$ bezeichnen wir

$$
\sigma(A)=\sum_{\substack{s \in A \\ \mu\{s\}>0}} \mu(\{s\}), \quad \tau(A)=\mu(A)-\sigma(A) .
$$

$\sigma$ und $\tau$ sind Borelsche Masse auf $\Delta$. Ist

$$
\begin{aligned}
& u_{\sigma}=\int_{\Delta_{1}} K_{s} d \sigma(s)=\sum_{\substack{\left.s \in \Delta_{1} \\
\mu(s)\right)>0}} \mu(\{s\}) K_{s}, \\
& u_{\tau}=\int_{\Delta_{1}} K_{s} d \tau(s)
\end{aligned}
$$

so ist $u_{\sigma}$ eine diskrete und $u_{\text {v }}$ eine total nichtdiskrete Funktion. Die Gleichheit

$$
u=u_{\sigma}+u_{\tau}
$$


stellt eine Zerlungen von $u$ in eine diskrete und eine total nichtdiskrete Funktion dar. Eine nichtnegative harmonische Funktion ist diskret dann und nur dann, wenn ihr kanonisches Mass in einer abzählbaren Menge konzentriert ist.

$R$ und $R^{\prime}$ werden von nun an zusammenhängend sein und $R \notin O_{G}$. Ist $R^{\prime}$ offen, so bezeichnen wir

$$
\begin{aligned}
& \Delta(f)=\left\{s \in \Delta \mid K_{s} \in H P(f)\right\}, \\
& \Delta_{1}(f)=\Delta(f) \cap \Delta_{1} .
\end{aligned}
$$

Ist $R^{\prime}$ kompakt, so setzen wir $\Delta(f)=\Delta_{1}(f)=\phi$. Wir wollen zeigen, dass $\Delta(f)$, $\Delta_{1}(f)$ Borelsche Mengen sind. Es sei $\left\{R_{n}^{\prime}\right\}$ eine normale Ausschöpfung von $R^{\prime}$ und $p_{0} \in f^{-1}\left(R_{1}^{\prime}\right)$. Wir bezeichnen

$$
\Gamma_{n}(\varepsilon)=\left\{s \in \Delta \mid \underset{f^{-1}\left(R^{\prime} n\right)}{I} K_{s}\left(p_{0}\right) \geq \varepsilon\right\}
$$

Es sei $\left\{s_{i}\right\}$ eine Folge aus $\Gamma_{n}(\varepsilon)$, die gegen $s_{0}$ konvergiert. Nach dem Satz 11 ist

$$
\underset{f^{-1}\left(R^{\prime} n\right)}{I} K_{s_{0}}\left(p_{0}\right) \geq \varlimsup_{i \rightarrow \infty} \underset{f^{-1}\left(R^{\prime} n\right)}{I} K_{s_{i}}\left(p_{0}\right) \geq \varepsilon
$$

Die Menge $\Gamma_{n}(\varepsilon)$ ist somit abgeschlossen. Die zu beweisende Behauptung wird aus der Gleichheit

$$
\Delta-\Delta(f)=\bigcup_{m=1}^{\infty} \bigcup_{n=1}^{\infty} \Gamma_{n}\left(\frac{1}{m}\right)
$$

folgen. Ist $s$ in $\Delta-\Delta(f)$ enthalten, so gehört $K_{s}$ der Klasse $H P(f)$ nicht an. Es gibt also ein relativkompaktes Gebiet $G^{\prime}$ derart, dass

$$
\underset{f^{-1}\left(G^{\prime}\right)}{I} K_{s} \neq 0
$$

ist. Es sei $\lambda$ ein Weg auf $R$, der $p_{0}$ mit einem Punkt $p_{1}$ von $f^{-1}\left(G^{\prime}\right)$ verbindet, für welchen

$$
\underset{f^{-1}\left(G^{\prime}\right)}{I} K_{s}\left(p_{1}\right)>0
$$

ist. Für ein genügend grosses $n$ ist

Aus dem Satz 12 folgt

$$
f(\lambda) \cup G^{\prime} \subset R_{n}^{\prime}
$$

$$
\underset{f^{-1}\left(G^{\prime}\right)}{I} K_{s} \leq\left(\underset{f^{-1}\left(E^{\prime} n\right)}{I} K_{s}\right) \circ \eta
$$


wo $\eta$ die identische Abbildung von $f^{-1}\left(G^{\prime}\right)$ in $f^{-1}\left(R_{n}^{\prime}\right)$ ist. Daraus ergibt sich, dass $\underset{f^{-1}\left(R^{\prime} n\right)}{I} K_{s}$ im Punkte $p_{0}$ nicht verschwinden kann und $s$ gehört der Menge $\bigcup_{m=1}^{\infty} \bigcup_{n=1}^{\infty} \Gamma_{n}\left(\frac{1}{m}\right)$ an. Da jeder Punkt dieser Menge offenbar der Menge $\Delta-\Delta(f)$ angehört, so ist die obige Gleichheit bewiesen.

HilfSSATZ 7. Es sei $u \in H P(R)$,

$$
u=\int_{\Delta} K_{s} d \mu(s)
$$

und $R^{\prime}$ offen. $u$ gehört der Klasse $H P(f)$ dann und nur dann an, wenn $\mu(\Delta-\Delta(f))=0$ ist. Ist $u^{\prime} \in H P\left(R^{\prime}\right)$ und

$$
u^{\prime} \circ f=\int_{\Delta_{1}} K_{s} d \mu(s)
$$

so ist

$$
I u^{\prime}=\int_{\Delta_{1}(f)} K_{s} d \mu(s)
$$

Ist $\mu(\Delta-\Delta(f))>0$, so ist $\mu\left(T_{n}(\varepsilon)\right)>0$ für wenigstens ein $n$ und ein $\varepsilon$. Ist

$$
v=\int_{\Gamma_{n}(\varepsilon)} K_{s} d \mu(s),
$$

so kann man eine Folge $\left\{u_{j}\right\}$ von Riemannschen Summen bilden,

$$
u_{j}=\sum_{i=1}^{k_{j}} K_{s_{j i}} \mu\left(A_{j i}\right) \quad\left(s_{j i} \in I_{n}^{\prime}(\varepsilon)\right)
$$

die gegen $v$ konvergiert. Dann ist nach dem Satz 11,

$$
\underset{f^{-1}\left(R^{\prime} n\right)}{I} v\left(p_{0}\right) \geq \varlimsup_{j \rightarrow \infty} \underset{f^{-1}\left(R^{\prime} n\right)}{I} u_{j}\left(p_{0}\right) \geqq \varepsilon \mu\left(\Gamma_{n}(\varepsilon)\right)>0 .
$$

$v$ gehört also der Klasse $H P(f)$ nicht an und, da $v \leq u$ ist, gehört auch $u$ der Klasse $H P(f)$ nicht an.

Es sei jetzt $\mu(\Delta-\Delta(f))=0$. Da $\Delta(f)$ eine Borelsche Menge und $\mu$ ein Borelsches Mass ist und $\Delta$ eine abzählbare Basis hat, so kann man eine nichtabnähmende Folge $\left\{A_{i}\right\}$ von abgeschlossenen Mengen finden, so dass $\mu(\Delta(f)-A)=0$ ist ([3], Seite 228), mit

$$
A=\bigcup_{i=1}^{\infty} A_{i} \subset \Delta(\dot{f})
$$


Dann ist $u=\int_{A} K_{s} d \mu(s)$. Wir bezeichnen

$$
u_{i}=\int_{A_{i}} K_{s} d \mu(s) \quad(i=1,2, \ldots) .
$$

$\left\{u_{i}\right\}$ ist eine nichtabnehmende Folge, die gegen $u$ konvergiert. Ist

$$
\underset{f^{-1}\left(g^{\prime}\right)}{I} u \neq 0
$$

für ein relativkompaktes Gebiet $G^{\prime}$ auf $R^{\prime}$, so ist laut des Satzes 11

$$
\underset{f^{-1}\left(G^{\prime}\right)}{I} u_{2} \neq 0
$$

für ein genügend grosses $i$. Wir können also vom Anfang an annehmen, dass $A$ abgeschlossen ist. Es sei $p$ ein Punkt von $f^{-1}\left(G^{\prime}\right)$, für welchen

$$
\underset{f^{-1}\left(G^{\prime}\right)}{I} u(p)>0
$$

ist, und $\left\{R_{n}\right\}$ eine normale Ausschöpfung von $R$. Wir bezeichnen mit $\eta_{n}$ die identische Abbildung von $f^{-1}\left(G^{\prime}\right) \cap R_{n}$ in $R_{n}$ und mit $\Gamma_{n}$ die Menge

$$
\Gamma_{n}=\left\{s \in A \mid I_{\eta_{n}} K_{S}(p) \geq \frac{1}{2 \mu(A)} \underset{f^{-1\left(G^{\prime}\right)}}{I} u(p)\right\}
$$

Da $A$ abgeschlossen ist, so folgt aus dem Satz 11, dass $\Gamma_{n}$ eine abgeschlossene Menge ist. Sind die Mengen $\Gamma_{n}$ nicht leer, so ist auch $\bigcap_{n=1}^{\infty} \Gamma_{n}$ nicht leer, da $A$ kompakt, und die Folge $\left\{\Gamma_{n}\right\}$ monoton ist. Für ein $s \in \bigcap_{n=1}^{\infty} \Gamma_{n}$ ist nach dem Satz 10,

$$
\underset{f^{-1}\left(G^{\prime}\right)}{I} K_{s}(p)=\lim _{n \rightarrow \infty} I_{\eta_{n}} K_{s}(p)>0,
$$

was der Beziehung $s \in \Delta(f)$ widerspricht. Für ein genügend grosses $n$ ist also $I_{n}$ leer. Es sei $\left\{u_{j}\right\}$ eine Folge von Riemannschen Summen

$$
u_{j}=\sum_{i=1}^{k_{i}} K_{s_{j i}} \mu\left(A_{j i}\right) \quad\left(s_{j i} \in A\right),
$$

die gleichmässig im Inneren von $R$ gegen $u$ konvergieren. Nach dem Beweis des Satzes 11 ist

$$
I_{\eta_{n}} u(p)=\lim _{\jmath \rightarrow \infty} I_{\eta_{n}} u_{j}(p)
$$

Aber

$$
I_{\eta_{n}} u_{j}(p)=\sum_{i=1}^{k_{j}} \mu\left(A_{j i}\right) I_{\eta_{n}} K_{s j i}(p) \leq \frac{1}{2 \mu(A)} \sum_{i=1}^{k_{j}} \mu\left(A_{j i}\right) \underset{f^{-1}\left(G^{\prime}\right)}{I} u(p)=\frac{1}{2} \underset{f^{-1_{\left(G^{\prime}\right)}}}{I} u(p)
$$


und man erhält die widersprechende Beziehung

$$
\underset{f^{-1}\left(\alpha^{\prime}\right)}{I} u(p) \leq I_{r_{n}} u(p) \leq \frac{1}{2} \underset{f^{-1}\left(\alpha^{\prime}\right)}{I} u(p) .
$$

Es ist also

$$
\underset{f^{-1}\left(G^{\prime}\right)}{I} u=0
$$

und $u \in H P(f)$.

Die Funktion $\int_{\Delta_{1}(f)} K_{s} d \mu(s)$ gehört also der Klasse $H P(f)$ an und, da sie offenbar nicht grösser als $u^{\prime} \circ f$ ist, so ist

$$
\int_{\Delta_{1}(f)} K_{s} d \mu(s) \leq I u^{\prime}
$$

Aus $I u^{\prime} \leq u^{\prime} \circ f$ und Hilfssatz a erhält man

$$
I u^{\prime}=\int_{\Delta_{1}} K_{s} \theta(s) d \mu(s),
$$

wo $\theta \leq 1$ ist. Da aber $I u^{\prime} \in H P(f)$, so folgt aus obigen Betrachtungen

$$
\begin{aligned}
& I u^{\prime}=\int_{\Delta_{1}(f)} K_{s} \theta(s) d \mu(s) \leq \int_{\Delta_{1}(f)} K_{s} d \mu(s), \\
& I u^{\prime}=\int_{\Delta_{1}(f)} K_{s} d \mu(s) .
\end{aligned}
$$

Aus diesem Hilfssatz ergibt sich $I 1=\omega\left(\Delta_{1}(f), R\right)$.

Wir nehmen jetzt an, dass $R^{\prime} \notin O_{G}$ und es sei $\Delta^{\prime}$ der ideale Rand von $R^{\prime}$ und $K_{s^{\prime}}^{\prime}$ die Funktion von Martin auf $R^{\prime}$. Für ein endliches Mass $\mu^{\prime}$ auf $\Delta^{\prime}$ werden wir mit $\mu^{\prime \circ} f$ das kanonische Mass der Funktion

$$
\left(\int_{\Delta^{\prime}} K_{s^{\prime}}^{\prime} d \mu^{\prime}\left(s^{\prime}\right)\right) \circ f
$$

bezeichnen. Aus

$$
\left(\int_{\Delta^{\prime}} K_{s^{\prime}}^{\prime} d \%_{R^{\prime}}\left(s^{\prime}\right)\right) \circ f=1 \circ f=1=\int_{\Delta_{1}} K_{S} d \chi_{R}(s)
$$

folgt $\chi_{R^{\prime}} \circ f=\psi_{R}$. Für einen Punkt $s^{\prime} \in \Delta^{\prime}$ bezeichnen wir mit $\delta_{s^{\prime}}$ das Mass auf $\Delta^{\prime}$

$$
\delta_{s^{\prime}}\left(A^{\prime}\right)=\left\{\begin{array}{l}
1 \text { für } A^{\prime} \ni s \\
0 \text { für } A^{\prime} \notin s .
\end{array}\right.
$$

Dann ist

$$
K_{\mathcal{S}^{\prime}}^{\prime} \circ f=\int_{\Delta_{1}} K_{s} d\left(\delta_{s^{\prime}} \circ f\right)(s)
$$


Es seien $X, X^{\prime}$ zwei lokalkompakte topologische Räume, $\mu^{\prime}$ ein Borelsches Mass auf $X^{\prime}$ und für jedes $x^{\prime} \in X^{\prime}$ sei $\mu_{x^{\prime}}$ ein Borelsches Mass auf $X$, so dass für jede Borelsche Menge $A$ aus $X$ die Funktion $x^{\prime} \rightarrow \mu_{x^{\prime}}(A)$ eine Borelsche Funktion ist. Wir setzen $\mu(A)=\int_{X^{\prime}} \mu_{x^{\prime}}(A) d \mu^{\prime}\left(x^{\prime}\right) ; \mu$ ist ein Borelsches Mass. Für jede nichtnegative Borelsche Funktion $\varphi$ auf $X$ ist

$$
x^{\prime} \rightarrow \int_{X} \varphi(x) d \mu_{x^{\prime}}(x)
$$

eine Borelsche Funktion auf $X^{\prime}$ und

$$
\int_{X_{1}}\left(\int_{X} \varphi(x) d \mu_{x^{\prime}}(x)\right) d \mu^{\prime}\left(x^{\prime}\right)=\int_{X} \varphi(x) d \mu(x) .
$$

Denn diese Formel ist erst für die charakteristischen Funktionen der Borelschen Mengen wahr und daraus folgert man sofort, dass sie für beliebige positive Borelsche Funktionen gilt.

Es sei $G$ eine offene Menge auf $R$ die den Punkt $p_{0}$ enthält. Die Funktion

$$
s^{\prime} \rightarrow \underset{G}{I}\left(K_{s^{\prime}}^{\prime} \circ f\right)\left(p_{0}\right)
$$

ist laut des Satzes 11 halbstetig und somit eine Borelsche Funktion. Aus

$$
H_{G}^{K^{\prime} s^{\prime} \circ f}\left(p_{0}\right)=\left(K_{s^{\prime}}^{\prime} \circ f\right)\left(p_{0}\right)-\underset{G}{I}\left(K_{s^{\prime}}^{\prime} \circ f\right)\left(p_{0}\right)
$$

(Hilfssatz 4) folgt, dass auch die Funktion

$$
s^{\prime} \rightarrow\left(K_{s^{\prime}}^{\prime} \circ f\right)_{R-G}^{*}\left(p_{0}\right)
$$

messbar ist. Es sei jetzt $A$ eine abgeschlossene Menge in $\Delta$ und

$$
A_{n}=\left\{p \in R \mid d(p, A) \leq \frac{1}{n}\right\},
$$

wo $d(p, A)$ die Entfernung zwischen $p$ und $A$ in der Metrik von Martin bezeichnet. Die Funktionen

$$
s^{\prime} \rightarrow\left(K_{s^{\prime}}^{\prime} \circ f\right)_{A_{n}}^{*}\left(p_{0}\right)
$$

bilden eine nicht zunehmende Folge und ihr Limes ist wieder eine Borelsche Funktion. Nach (4) ist

$$
\lim _{n \rightarrow \infty}\left(K_{s^{\prime}}^{\prime} \circ f\right)_{A_{n}}^{*}\left(p_{0}\right)=\int_{A} K_{s}\left(p_{0}\right) d\left(\delta_{s^{\prime}} \circ f\right)(s)=\left(\delta_{s^{\prime}} \circ f\right)(A) .
$$

Es sei $\Phi$ die Klasse der Borelschen Mengen $A \subset \Delta$, für die die Funktion 
$s^{\prime} \rightarrow\left(\delta_{s^{\prime}} \circ f\right)(A)$ messbar ist. $\emptyset$ enthält also die abgeschlossenen Mengen. Es seien $\left\{A_{i}\right\}$ eine monotone Folge von Mengen aus $\emptyset$. Aus

$$
\left(\delta_{s^{\prime}} \circ f\right)\left(\lim _{i \rightarrow \infty} A_{i}\right)=\lim _{i \rightarrow \infty}\left(\delta_{s^{\prime}} \circ f\right)\left(A_{i}\right)
$$

sieht man, dass $\Phi$ eine monotone Klasse ist ([3] Seite 26). Daraus folgt, dass $\Phi$ alle Borelschen Mengen enthält.

Hilfssatz 8. Es sei $\mu^{\prime}$ ein endliches Borelsches Mass auf $4^{\prime}$. Dann ist

$$
\int_{\Delta^{\prime}}\left(\int_{A} K_{s} d\left(\delta_{s^{\prime}} \circ f\right)(s)\right) d \mu^{\prime}\left(s^{\prime}\right)=\int_{A} K_{S} d\left(\mu^{\prime} \circ f\right)(s) .
$$

Nach obigen Bemerkungen erfüllen die Masse $\delta_{\mathcal{S}^{\prime}} \circ f$ die Bedingungen der Formel (5), und somit ist

$$
\int_{\Delta^{\prime}}\left(\int_{A} K_{s} d\left(\delta_{s^{\prime}} \circ f\right)(s)\right) d \mu^{\prime}\left(s^{\prime}\right)=\int_{A} K_{s} d \mu(s)
$$

wo

$$
\mu(B)=\int_{\Delta^{\prime}}\left(\delta_{s^{\prime}} \circ f\right)(B) d \mu^{\prime}\left(s^{\prime}\right)
$$

ist. Für $A$ gleich $\Delta_{1}$ ist also laut der Definition von $\mu^{\prime} \circ f$

$$
\begin{gathered}
\int_{\Delta^{\prime}}\left(\int_{\Delta_{1}} K_{s} d\left(\delta_{s^{\prime}} \circ f\right)(s)\right) d \mu^{\prime}\left(s^{\prime}\right)=\int_{\Delta^{\prime}}\left(K_{s^{\prime}}^{\prime} \circ f\right) d \mu^{\prime}\left(s^{\prime}\right) \\
=\left(\int_{\Delta^{\prime}} K_{s^{\prime}}^{\prime} d \mu^{\prime}\left(s^{\prime}\right)\right) \circ f=\int_{\Delta_{1}} K_{s} d\left(\mu^{\prime} \circ f\right)(s),
\end{gathered}
$$

und somit ist

$$
\int_{\Delta_{1}} K_{s} d\left(\mu^{\prime} \circ f\right)(s)=\int_{\Delta_{1}} K_{s} d \mu(s) .
$$

Aus der Eindeutigkeit der kanonischen Masse folgt $\mu=\mu^{\prime} \circ f$, was zu beweisen war.

SATZ 14. Ist $R^{\prime} \notin O_{G}$ und

$$
u^{\prime}=\int_{\Delta^{\prime}} K_{s^{\prime}}^{\prime} d \mu^{\prime}\left(s^{\prime}\right) \in H P\left(R^{\prime}\right)
$$

so ist

$$
I u^{\prime}=\int_{\Delta_{1}(f)} K_{s} d\left(\mu^{\prime} \circ f\right)(s)=\int_{\Delta^{\prime}} I K_{s^{\prime}}^{\prime} d \mu^{\prime}\left(s^{\prime}\right) .
$$

Ist für alle $s^{\prime} \in \Delta^{\prime}$, bis auf eine Borelsche Menge vom $\mu^{\prime}$-Masse Null, 


$$
I K_{S^{\prime}}^{\prime}=\int_{A} K_{S} d\left(\delta_{s^{\prime}} \circ f\right)(s),
$$

wo $A \subset \Delta_{1}$ eine Borelsche Menge bedeutet, so ist

$$
I u^{\prime}=\int_{A} K_{s} d\left(\mu^{\prime} \circ f\right)(s)
$$

Da nach der Definition von $\mu^{\prime} \circ f$

$$
u^{\prime} \circ f=\int_{\Delta_{1}} K_{s} d\left(\mu^{\prime} \circ f\right)(s)
$$

ist, so folgt aus dem Hilfssatz 7

Insbesondere ist

$$
I u^{\prime}=\int_{\Delta_{1}(f)} K_{s} d\left(\mu^{\prime} \circ f\right)(s) .
$$

$$
I K_{S^{\prime}}^{\prime}=\int_{\Delta_{1}(f)} K_{s} d\left(\delta_{s^{\prime}} \circ f\right)(s)
$$

Aus dem Hilfssatz 8 haben wir

$$
\int_{\Delta^{\prime}} I K_{s^{\prime}}^{\prime} d_{\iota^{\prime}}(s)=\int_{\Delta^{\prime}}\left(\int_{\Delta_{1}(f)} K_{s} d\left(\delta_{s^{\prime}} \circ f\right)(s)\right) d_{\ell^{\prime}}\left(s^{\prime}\right)=\int_{\Delta_{1}(f)} K_{s} d\left(\iota^{\prime} \circ f\right)(s)=I u^{\prime} .
$$

Die letzte Behauptung ergibt sich aus

$$
I u^{\prime}=\int_{\Delta^{\prime}} I K_{s^{\prime}}^{\prime} d \mu^{\prime}\left(s^{\prime}\right)=\int_{\Delta^{\prime}}\left(\int_{A} K_{s} d\left(\delta_{s^{\prime}} \circ f\right)(s)\right) d \mu^{\prime}\left(s^{\prime}\right)=\int_{A} K_{s} d\left(\mu^{\prime} \circ f\right)(s) .
$$

Wir werden mit $\Delta^{\prime}(f), \Delta_{1}^{\prime}(f)$ die Mengen

$$
\Delta^{\prime}(f)=\left\{s^{\prime} \in \Delta^{\prime} \mid I K_{s^{\prime}}^{\prime} \neq 0\right\}, \quad \Delta_{1}^{\prime}(f)=\Delta_{1}^{\prime} \cap \Delta^{\prime}(f)
$$

bezeichnen. Es ist offenbar

$$
\Delta^{\prime}(f)=\bigcup_{n=1}^{\infty}\left\{s^{\prime} \in \Delta^{\prime} \mid I K_{s^{\prime}}^{\prime}\left(p_{0}\right) \geq \frac{1}{n}\right\}
$$

Daraus erkennt man, dass $\Delta^{\prime}(f)$ eine Menge vom Typus $F_{\sigma}$ ist. Ist $G$ eine offene Menge auf $R$, so setzen wir $\Delta_{1}(G)=\left\{s \in \Delta_{1} \mid I_{G} K_{s} \neq 0\right\}$. Aus $\Delta_{1}(G)$ $=\bigcup_{i} \Delta_{1}\left(G_{i}\right)$, wo $G_{i}$ die Komponenten von $G$ sind, sieht man, dass auch $\Delta_{1}(G)$ eine Borelsche Menge ist.

HilfsSATZ 9. Ist $u \in H P(f)$ und

$$
E u=\int_{\Delta^{\prime}} K_{s^{\prime}}^{\prime} d \mu^{\prime}\left(s^{\prime}\right)<\infty
$$


so ist $\mu^{\prime}\left(\Delta^{\prime}-\Delta^{\prime}(f)\right)=0$.

Wir setzen

$$
u^{\prime}=\int_{\Delta^{\prime}-\Delta^{\prime}(f)} K_{s^{\prime}}^{\prime} d \mu^{\prime}\left(s^{\prime}\right) \leq E u .
$$

Nach Satz 14 ist $I u^{\prime}=0$ und nach Hilfssatz 3 ist

$$
u^{\prime}=E I u^{\prime}=0, \quad \mu^{\prime}\left(\Delta^{\prime}-\Delta^{\prime}(f)\right)=u^{\prime}\left(p_{0}^{\prime}\right)=0 .
$$

SATZ 15. Ist

$$
u^{\prime}=\int_{\Delta^{\prime} 1} K_{s^{\prime}}^{\prime} d \mu^{\prime}\left(s^{\prime}\right) \in H P\left(R^{\prime}\right),
$$

so ist auch

$$
E I u^{\prime}=\int_{\Delta^{\prime}(f)} K_{s^{\prime}}^{\prime} d \mu^{\prime}\left(s^{\prime}\right)
$$

Es sei $u^{\prime}=u_{1}^{\prime}+u_{2}^{\prime}$ die im Hilfssatz 3 eingeführte Zerlegung und

$$
u_{i}^{\prime}=\int_{\Delta^{\prime} 1} K_{s^{\prime}}^{\prime} d \mu_{l}^{\prime}\left(s^{\prime}\right) \quad(i=1,2)
$$

Da nach Satz 14

$$
0=I u_{2}^{\prime}=\int_{\Delta_{1}} I K_{s^{\prime}}^{\prime} d \mu_{2}^{\prime}\left(s^{\prime}\right)
$$

ist, so haben wir $\mu_{2}^{\prime}\left(\Delta_{1}^{\prime}(f)\right)=0 . \quad \mathrm{Da} u_{1}^{\prime}=E I u^{\prime}$ ist, so gilt, nach Hilfssatz 9, $\mu_{1}^{\prime}\left(\Delta_{1}^{\prime}-\Delta_{1}^{\prime}(f)\right)=0$. Daraus und aus $\mu^{\prime}=\mu_{1}^{\prime}+\mu_{2}^{\prime}$ folgt

$$
E I u^{\prime}=u_{1}^{\prime}=\int_{\Delta_{1}^{\prime}(f)} K_{s^{\prime}}^{\prime} d \mu^{\prime}\left(s^{\prime}\right) .
$$

Aus diesem Satz ergibt sich insbesondere

$$
\begin{aligned}
& E I \omega\left(A^{\prime}, R^{\prime}\right)=\int_{A^{\prime} \cap \Delta_{1}^{\prime}(f)} K_{s^{\prime}}^{\prime} d \%_{R^{\prime}}\left(s^{\prime}\right)=\omega\left(A^{\prime} \cap \Delta_{1}^{\prime}(f), R^{\prime}\right), \\
& E I 1=\omega\left(\Delta_{1}^{\prime}(f), R^{\prime}\right) .
\end{aligned}
$$

Hilfssatz 10. Für zwei offene Mengen $G_{1}, G_{2}$ auf $R$ ist

$$
\Delta_{1}\left(G_{1}\right) \cap \Delta_{1}\left(G_{2}\right)=\Delta_{1}\left(G_{1} \cap G_{2}\right) .
$$

Es ist offenbar $\Delta_{1}\left(G_{1} \cap G_{2}\right) \subset \Delta_{1}\left(G_{1}\right) \cap \Delta_{1}\left(G_{2}\right)$. Es sei jetzt $s \in \Delta_{1}\left(G_{1}\right) \cap \Delta_{1}\left(G_{2}\right)$. Dann ist

$$
\underset{G_{i}}{I} K_{s} \neq 0 \quad(i=1,2)
$$

und nach Folgesatz 2 ist $G_{1} \cap G_{2} \neq \phi$, 
und

$$
\underset{G_{1} \cap G_{2}}{I} K_{s} \neq 0
$$

$$
\Delta_{1}\left(G_{1}\right) \cap \Delta_{1}\left(G_{2}\right) \subset \Delta_{1}\left(G_{1} \cap G_{2}\right) .
$$

SATZ 15'. Ist

$$
u=\int_{\Delta_{1}} K_{s} d \mu(s) \in H P(R)
$$

und $G$ eine offene Menge von $R$, so ist

$$
\underset{G Q}{E I} u=\int_{\Delta_{1}(G)} K_{s} d \mu(s)
$$

Es seien $G_{i}$ die Komponenten von $G$. Nach Satz 15 ist

$$
\underset{G_{i} G_{i}}{E I} u=\int_{\Delta_{1}\left(G_{i}\right)} K_{s} d \mu(s) .
$$

Daraus und aus Hilfssatz 10 folgert man

$$
\sum_{i} \underset{G_{i}}{E} I u=\sum_{i} \int_{\Delta_{1}\left(G_{i}\right)} K_{s} d \mu(s)=\int_{\underset{i}{\cup \Delta_{1}\left(G_{i}\right)}} K_{s} d \mu(s)=\int_{\Delta_{1}(G)} K_{s} d \mu(s)
$$

Es sei $\eta$ die identische Abbildung von $G$ in $R$. Es ist

$$
I_{a} u \leq(\sum_{i} \underbrace{}_{G_{i}} I u) \circ \eta
$$

und deshalb

$$
\underset{G}{E} I_{G} u \leq \sum_{i} \underset{G_{i} G_{i}}{E} I u .
$$

Aus $\underset{G_{i}}{E} I_{G_{i}} u \leq \underset{G G}{E I} u$ und $\left(\underset{G_{i} G_{i}}{E I} u\right) \wedge\left(\underset{G_{j} G_{j}}{E I} u\right)=0$ für $i \neq j$, folgt

$$
\sum_{i} \underset{G_{i} G_{i}}{E} I_{G_{i}} u=\underset{i}{\bigvee} \underset{G_{i} G_{i}}{E} I \underset{G \in}{E} I u
$$

Es sei $\sigma$ eine endliche totaladditive Mengenfunktion,

$$
u=\int_{\Delta_{1}} K_{s} d \sigma(s), \quad G_{0}=\{p \in R \mid u(p)>0\}
$$

und $\eta$ die identische Abbildung von $G_{0}$ in $R$. Nach b) Satz 6 ist $u^{\circ} \eta \in H P(\eta)$. Es ist offenbar

$\mathrm{Da}$

$$
u \vee 0=\underset{G_{0}}{E}(u \circ \eta)=\underset{G_{0}}{E} \underset{G_{0}}{I} \underset{G_{0}}{E}(u \circ \eta)
$$

$$
u \vee 0=\int_{\Delta_{1}} K_{s} d \sigma^{+}
$$


ist, so folgt, aus dem Satz $15^{\prime}, \sigma^{+}\left(\Delta_{1}-\Delta_{1}\left(G_{0}\right)\right)=0$. Ähnlicherweise beweist man, dass $\sigma^{-}\left(\Delta_{1}-\Delta_{1}\left(R-\bar{G}_{0}\right)\right)=0$ ist. Daraus folgt, dass $\Delta_{1}\left(G_{0}\right)$ eine positive und $\Delta_{1}\left(R-\bar{G}_{0}\right)$ eine negative Menge für $\sigma$ ist.

Hilfssatz 11. Es sei u eine Differenz von zwei nichtnegativen harmonischen Funktionen auf $R$ :

$$
u=\int_{\Delta_{1}} K_{s} \theta(s) d \chi(s)+\int_{\Delta_{1}} K_{s} d \sigma_{0}(s),
$$

wobei o orthogonal zu $\chi$ ist. Setzt man

so ist

$$
A_{\alpha}=\left\{s \in \Delta_{1} \mid \theta(s)>\alpha\right\}, \quad G_{\alpha}=\{p \in R \mid u(p)>\alpha\},
$$

$$
A_{\alpha} \subset \Delta_{1}\left(G_{\alpha}\right):[\%]
$$

und $\Delta_{1}\left(G_{\alpha}\right)$ ist eine positive Menge für $\sigma_{0}$. Ist für ein $\alpha\left\{s \in \Delta_{1} \mid \theta(s)=\alpha\right\}$ vom harmonischen Masse Null, so ist $\Delta_{1}-\Delta_{1}\left(G_{\alpha}\right)-\Delta_{1}\left(R-\bar{G}_{\alpha}\right)$ auch vom harmonischen Masse Null.

Es genügt den Hilfssatz für $\alpha=0$ zu beweisen, denn wir können $u-\alpha$ an Stelle von $u$ betrachten. Es sei $\Gamma_{0}, \Gamma_{1}$ eine Zerlegung von $\Delta_{1}$, für die $\chi\left(\Gamma_{0}\right)=0$, $\left|\sigma_{0}\right|\left(\Gamma_{1}\right)=0$ ist und

$$
u=\int_{\Delta_{1}} K_{s} d \sigma(s), \quad \sigma(A)=\int_{A} \theta(s) d \chi(s)+\sigma_{0}(A) .
$$

Nach obigen Bemerkungen ist $\Delta_{1}\left(G_{0}\right)$ eine positive Menge für $\sigma$. Ist $A \subset \Delta_{1}\left(G_{0}\right)$, so ist

$$
\sigma_{0}(A)=\sigma_{0}\left(A \cap \Gamma_{0}\right)=\sigma\left(A \cap \Gamma_{0}\right) \geq 0 .
$$

Daraus folgt, dass $\Delta_{1}\left(G_{0}\right)$ eine positive Menge für $\sigma_{0}$ ist. Es ist

$$
\begin{aligned}
0 & \leqq \int_{A_{0} \cap\left(\Delta_{1}-\Delta_{1}\left(G_{0}\right)\right)} \theta(s) d \%(s)=\int_{\Delta_{0} \cap\left(\Delta_{1}-\Delta_{1}\left(\left(_{0}\right)\right) \cap \Gamma_{1}\right.} \theta(s) d \chi(s) \\
& =\sigma\left(A_{0} \cap\left(\Delta_{1}-\Delta_{1}\left(G_{0}\right)\right) \cap \Gamma_{1}\right)=-\sigma^{-}\left(A_{0} \cap\left(\Delta_{1}-\Delta_{1}(G)\right) \cap I_{1}\right) \leqq 0
\end{aligned}
$$

und somit

$$
\chi\left(A_{0} \cap\left(\Delta_{1}-\Delta_{1}\left(G_{0}\right)\right)\right)=0, A_{0} \supset \Delta_{1}\left(G_{0}\right):[\chi] .
$$

Wir nehmen jetzt an, dass die Menge $\left\{s \in \Delta_{1} \mid \theta(s)=\alpha\right\}$ vom harmonischen Masse Null ist und es sei

$$
A_{\alpha}^{-}=\left\{s \in \Delta_{1} \mid \theta(s)<\alpha\right\} \subset \Delta_{1}\left(R-\bar{G}_{\alpha}\right):[\%] .
$$

Dann ist $\Delta_{1}-A_{\alpha}-A_{\alpha}^{-}$vom harmonischen Masse Null. Da, nach dem Hilfssatz $10, \Delta_{1}\left(G_{\alpha}\right), \Delta_{1}\left(R-\bar{G}_{\alpha}\right)$ punktfremd sind, ist 


$$
\chi\left(\Delta_{1}\left(G_{\alpha}\right) \cup \Delta_{1}\left(R-\bar{G}_{\alpha}\right)\right)=\chi\left(\Delta_{1}\left(G_{\alpha}\right)\right)+\chi\left(\Delta_{1}\left(R-\bar{G}_{\alpha}\right)\right) \geq \chi\left(A_{\alpha}\right)+\chi\left(A_{\alpha}^{-}\right)=1
$$

und somit ist $\Delta_{1}-\Delta_{1}\left(G_{\alpha}\right)-\Delta_{1}\left(R-\bar{G}_{\alpha}\right)$ vom harmonischen Masse Null.

\section{Die Hauptsätze}

Es ist wichtig den idealen Rand von Martin auch für Riemannsche Flächen $R^{\prime}$ ohne Greensche Fnnktion einzuführen. Es sei $G^{\prime}$ eine Kreisscheibe auf $R^{\prime}$ und $R=R^{\prime}-\bar{G}^{\prime}$. $R$ hat eine Greensche Funktion und somit einen idealen Rand $\Delta$. Es sei $\Delta_{G^{\prime}}$ die Teilmenge jener Punkte von $\Delta$ die nicht auf dem Rand von $G^{\prime}$ liegen. Wir bezeichnen $\hat{R}_{G^{\prime}}^{\prime}=R^{\prime} \cup \Delta_{G^{\prime}}$ und führen auf $\hat{R}_{G^{\prime}}^{\prime}$ diejenige Topologie ein, für welche die identische Abbildung von $\hat{R}$ in $\hat{R}_{G^{\prime}}^{\prime}-G^{\prime}$ ein Homeomorphismus ist. Man kann leicht zeigen, dass der topologische (metrisierbare) Raum $\hat{R}_{G^{\prime}}^{\prime}$ von $G^{\prime}$ nicht abhängt [13]. Deshalb werden wir einfach $\Delta^{\prime}$ und $\hat{R}^{\prime}$ an Stelle von $\Delta_{Q^{\prime}}$ und $\hat{R}_{G^{\prime}}^{\prime}$ schreiben. $\quad \Delta^{\prime}$ heisst der ideale Rand von $R^{\prime}$. Ähnlicherweise führt man die Menge $\Delta_{1}^{\prime}$ ein. Ist $R^{\prime}$ kompakt, so ist offenbar $\Delta^{\prime}$ leer.

Eine (nicht unbedingt Borelsche) Menge $\hat{A}^{\prime}$ auf $\hat{R}^{\prime}$ heisst polar, falls für jede Kreisscheibe $G^{\prime}$ auf $R^{\prime}$ eine Funktion $S^{\prime} \in S P\left(R^{\prime}-\bar{G}^{\prime}\right)$ existiert, $S^{\prime}+\infty$, für die in jedem Punkt $\hat{q}^{\prime} \in \hat{A}^{\prime}-G^{\prime}$

$$
\lim _{p^{\prime} \rightarrow \hat{q}^{\prime}} S^{\prime}\left(p^{\prime}\right)=+\infty
$$

gilt. Eine Menge $M^{\prime}$ aus $R^{\prime}$ ist dann und nur dann polar, wenn sie eine Nulle äussere Kapazität hat; insbesondere ist ein Punkt aus $R^{\prime}$ eine polare Menge. Hat $R^{\prime}$ eine Greensche Funktion und ist $\hat{A}^{\prime}$ polar auf $\hat{R}^{\prime}$, so kann man leicht beweisen, dass sogar eine Funktion $S^{\prime} \in S P\left(R^{\prime}\right)$ zu finden ist, die die obige Beziehung erfüllt. Eine Teilmenge einer polaren Menge und die Vereinigung abzählbar vieler polarer Mengen sind polar.

Es sei $\Gamma$ eine offene Menge aus $A^{11}$. Dann gibt es eine Funktion $S_{\Gamma} \in S P(R)$ für die

$$
\lim _{p \rightarrow \Gamma} S_{\Gamma}(p) \geq 1, \quad S_{\Gamma}\left(p_{0}\right) \leq 2 \chi(\Gamma)
$$

ist. In der Tat, es sei $\left\{\varepsilon_{n}\right\}$ eine abnehmende Folge positiver Zahlen, die gegen Null konvergieren, so dass die Menge der Punkte von $\Gamma$, deren Entfernung (in der Metrik von Martin) von $\Delta-\Gamma$ gleich $\varepsilon_{n}$ ist, vom harmonischen Masse Null

11) $R \notin O_{G}$. 
ist. Es sei $\Gamma_{n}$ die (abgeschlossene) Menge der Punkte aus $\Gamma$, deren Entfernung von $\Delta-\Gamma$ nicht kleiner als $\varepsilon_{n}$ ist. Dann haben wir

$$
\chi\left(\Gamma_{n}-\Gamma_{n-1}\right)=\chi\left(\overline{\Gamma_{n}-\Gamma_{n-1}}\right) \quad\left(\Gamma_{0}=\phi\right) .
$$

Nach (4) kann man eine offene Menge $\hat{G}_{n}$ finden, $\hat{G}_{n} \supset{\overline{\Gamma_{n}}-I_{n-1}}_{\text {, für die }}$

$$
1 \stackrel{*}{\overline{\hat{G}}_{n \cap R}}\left(p_{0}\right) \leq 2 \omega\left(p_{0} ; \overline{\Gamma_{n}-\Gamma_{n-1}}\right)=2 \chi\left(\overline{\Gamma_{n}-\Gamma_{n-1}}\right)
$$

ist. Wir setzen

$$
S_{\Gamma}=\sum_{n=1}^{\infty} 1_{\hat{\hat{\alpha}}_{n} \cap R}^{*}
$$

Es ist

$$
S_{\Gamma}\left(p_{0}\right) \leq 2 \sum_{n=1}^{\infty} \chi\left(\overline{\Gamma_{n}-\Gamma_{n-1}}\right)=2 \chi(\Gamma)
$$

und $\lim _{p \rightarrow \Gamma} S_{\Gamma}(p) \geq 1$. Es sei $A \subset \Delta$ eine Menge vom harmonischen Masse Null und $\left\{\Gamma^{n}\right\}$ eine nichtzunehmende Folge von offenen Mengen in $\Delta$, die $A$ enthalten und

$$
\sum_{n=1}^{\infty} \chi\left(\Gamma^{n}\right)<\infty
$$

ist. Dann ist

$$
S=\sum_{n=1}^{\infty} S_{\Gamma^{n}}+\infty,
$$

$S \in S P(R)$ und

$$
\lim _{p \rightarrow A} S(p)=+\infty
$$

Jede Menge aus 4 vom harmonischen Masse Null ist polar. Insbesondere ist die Menge $\Delta-\Delta_{1}$ polar. Jeder Punkt $s \in \Delta_{1}$, für welchen $K_{s}$ nicht beschränkt ist, bildet eine polare Menge. Der ganze ideale Rand einer Riemannscher Fläche $R^{\prime} \in O_{G}$ ist polar, da er auf dem idealen Rand von $R^{\prime}-G^{\prime}$ eine Menge vom harmonischen Masse Null ist. Umgekehrt ist jede polare Menge aus 4 vom harmonischen Masse Null. Es sei $A \subset \Delta$ eine polare Menge, $S \in S P(R), S \neq+\infty$, $S\left(p_{0}\right) \neq+\infty$ und

$$
\lim _{p \rightarrow \hat{q} \in A} S(p)=\infty
$$

Wir bezeichnen

$$
\begin{aligned}
& G_{\alpha}=\{p \in R \mid S(p)>\alpha\}, \\
& G(s, \varepsilon)=\{p \in R \mid d(p, s)<\varepsilon\} .
\end{aligned}
$$


Es gibt offenbar für jedes $s \in A$ ein $\varepsilon>0$, so dass $G(s, \varepsilon) \subset G_{\alpha}$ und somit $\Delta_{1}(G(s, \varepsilon)) \subset \Delta_{1}\left(G_{\alpha}\right)$ ist. $s$ gehört aber der Menge $\Delta_{1}(G(s, \varepsilon))$ an, da

$$
\underset{G(s, \varepsilon)}{I} K_{s} \neq 0
$$

ist, wie am Anfang des Abschnitts III bewiesen wurde. Es ist also $A \subset \Delta_{1}\left(G_{a}\right)$. Aus

auf $G_{\alpha}$, folgt

$$
\underset{G \alpha}{I} 1 \leq 1 \leq \frac{1}{\alpha} S
$$

$$
\underset{G \alpha \alpha}{E} \underset{G \alpha}{I} 1 \leq \frac{1}{\alpha} S
$$

Nach Satz $15^{\prime}$ ist dann $\omega\left(\Delta_{1}\left(G_{\alpha}\right)\right) \leq \frac{1}{\alpha} S$. Daraus ergibt sich

$$
\lim _{\alpha \rightarrow \infty} \chi\left(\Delta_{1}\left(G_{\alpha}\right)\right)=\lim _{\alpha \rightarrow \infty} \omega\left(p_{0} ; \Delta_{1}\left(G_{\alpha}\right)\right) \leq \lim _{\alpha \rightarrow \infty} \frac{1}{\alpha} S\left(p_{0}\right)=0,
$$

was $\mathrm{zu}$ beweisen war.

Für jeden Punkt $s \in \Delta_{1}$ bezeichnen wir

$$
\hat{M}_{f}(s)=\hat{M}(s)=\bigcap_{\Delta_{1}(G) \ni s} \overline{f(G)} ;{ }^{12)}
$$

dabei ist $\overline{f(G)}$ die abgeschlossene Hülle von $f(G)$ in $\hat{R}^{\prime}$. Der Durchschnitt endlich vieler Mengen $\overline{f(G)}$ mit $\Delta_{1}(G) \ni s$ ist nicht leer, da nach Hilfssatz 10

$$
\bigcap_{i=1}^{n} \overline{f\left(G_{i}\right)} \supset f\left(\bigcap_{i=1}^{n} G_{i}\right) \neq \phi
$$

ist. Daraus und aus der Tatsache, dass $\hat{R}^{\prime}$ kompakt ist, folgt, dass $\hat{M}(s)$ eine abgeschlossene nichtleere Menge ist.

a) Für jede offene Menge $\hat{G}^{\prime}$ die $\hat{M}(s)$ enthält, ist $s \in \Delta_{1}\left(f^{-1}\left(\hat{G}^{\prime} \cap R^{\prime}\right)\right)$. In der Tat, wäre, für jede offene Menge $G$, für die $s \in \Delta_{1}(G)$ ist, $f(G) \nsubseteq \hat{G}^{\prime}$, so bildet $\left\{\overline{f(G)}-\hat{G}^{\prime} \mid \Delta_{1}(G) \ni s\right\}$ eine Klasse von nichtleeren abgeschlossenen Mengen. Für $\Delta_{1}\left(G_{i}\right) \ni s(i=1,2, \ldots, n)$ ist nach Hilfssatz $10 \Delta_{1}\left(\bigcap_{i=1}^{n} G_{i}\right) \ni s$ und somit

$$
\bigcap_{i=1}^{n}\left(\overline{f\left(G_{i}\right)}-\hat{G}^{\prime}\right)=\left(\bigcap_{i=1}^{n} \overline{f\left(G_{i}\right)}\right)-\hat{G}^{\prime} \supset f\left(\bigcap_{i=1}^{n} G_{i}\right)-\hat{G}^{\prime} \neq \phi .
$$

Daraus folgt, wie oben

12) Für die Definition von $\hat{M}_{f}$ genügt, dass $\hat{R^{\prime}}$ ein topologischer Raum sei. 


$$
\bigcap_{\Delta_{1}(G) \ni s}\left(\overline{f(G)}-\hat{G}^{\prime}\right) \neq \phi .
$$

Diese Menge ist einerseits in $\hat{M}(s)$, andererseits in $\hat{R}^{\prime}-\hat{G}^{\prime}$ enthalten, was widersprechend ist. Es gibt also ein $G, \Delta_{1}(G) \ni s$ mit $f(G) \subset \hat{G}^{\prime}$. Aus $G \subset f^{-1}(f(G)) \subset f^{-1}\left(\hat{G}^{\prime} \cap R^{\prime}\right)$ folgt $s \in \Delta_{1}\left(f^{-1}\left(\hat{G}^{\prime} \cap R^{\prime}\right)\right)$.

b) Die Menge $\hat{M}(s)$ ist zusammenhängend, denn im entgegengesetzten Falle könnte man zwei punktfremde offene Mengen $\hat{G}_{1}^{\prime}, \hat{G}_{2}^{\prime}$ derart finden, dass $\hat{M}(s) \subset \hat{G}_{1}^{\prime} \cup \hat{G}_{2}^{\prime}, \hat{M}(s) \cap \hat{G}_{i}^{\prime} \neq \phi(i=1,2)$ ist. Aus obigen Betrachtungen folgt $s \in A_{1}\left(f^{-1}\left(\hat{G}_{1}^{\prime} \cap R^{\prime}\right) \cup f^{-1}\left(\hat{G}_{2}^{\prime} \cap R^{\prime}\right)\right)$. Da aber $f^{-1}\left(\hat{G}_{1}^{\prime} \cap R^{\prime}\right) \cap f^{-1}\left(\hat{G}_{2}^{\prime} \cap R^{\prime}\right)=\phi$ ist, so muss $s$ wenigstens einer Menge $\Delta_{1}\left(f^{-1}\left(\hat{G}_{i}^{\prime} \cap R^{\prime}\right)\right)$ angehören. Ist das der Fall z.B. für $i=1$, so ist $\hat{M}(s) \subset \overline{\hat{G}}_{1}^{\prime}$, entgegen der Voraussetzung, dass

$$
\hat{M}(s) \cap \hat{G}_{2}^{\prime} \neq \phi
$$

ist.

c) Aus $s \in \Delta_{1}-\Delta_{1}(f)$ folgt $\hat{M}(s) \subset R^{\prime}$ und umgekehrt. Ist $R^{\prime}$ kompakt, so ist diese Bahauptung evident, so dass wir annehmen können, dass $R^{\prime}$ offen ist. Ist $s \in \Delta_{1}-\Delta_{1}(f)$, so ist $K_{s} \notin H P(f)$, und es gibt ein relativkompaktes Gebiet $G^{\prime} \subset R^{\prime}$, so dass $s \in \Delta_{1}\left(f^{-1}\left(G^{\prime}\right)\right)$. Es ist also $\hat{M}(s) \subset \overline{G^{\prime}} \subset R^{\prime} . \quad$ Ist $\hat{M}(s) \subset R^{\prime}$, so existiert ein relativkompaktes Gebiet $G^{\prime} \subset R^{\prime}$, das die Menge $\hat{M}(s)$ enthält. Dann ist nach a)

$$
\underset{f^{-1}\left(\alpha^{\prime}\right)}{I} K_{s} \neq 0
$$

und $s \in \Delta_{1}-\Delta_{1}(f)$.

d) Es gibt einen Weg $\lambda$ auf $R$ mit dem Endpunkt in s, so dass die Menge der Limespunkte von $f$ auf $\lambda$ (cluster set) mit $\hat{M}(s)$ zusammenfällt und

$$
\lim _{\lambda \ni p \rightarrow s} K_{s}(p)=\sup K_{s}
$$

ist. Es sei $G(s, 1 / n)$ (bzw. $G^{\prime}(\hat{M}(s), 1 / n)$ ) die Menge der Punkte von $R$ (bzw. $R^{\prime}$ ), deren Entfernung von $s$ (bzw. $\hat{M}(s)$ ) kleiner als $1 / n$ ist und

$$
G_{\alpha_{n}}=\left\{p \in R \mid K_{s}(p)>\alpha_{n}\right\},
$$

wo $\alpha_{n} \uparrow \sup K_{\mathrm{s}}$ ist. Am Anfang des Abschnittes III wurde gezeigt, dass

$$
\underset{G(s, 1 / n)}{I} K_{s} \neq 0, \quad \underset{G \alpha_{n}}{I} K_{s} \neq 0
$$

und somit $s \in \Delta_{1}(G(s, 1 / n)), s \in \Delta_{1}\left(G_{\alpha_{n}}\right)$ ist. Aus a) folgt $s \in \Delta_{1}\left(f^{-1}\left(G^{\prime}(\hat{M}(s), 1 / n)\right)\right)$. 
Laut des Hifssatzes 10 ist dann $s \in A_{1}\left(G(s, 1 / n) \cap G_{\alpha_{n}} \cap f^{-1}\left(G^{\prime}(\hat{M}(s), 1 / n)\right)\right)$. Es sei $D_{n}$ diejenige Komponente (Folgesatz 2) von $G(s, 1 / n) \cap G_{\alpha_{n}} \cap f^{-1}\left(G^{\prime}(\hat{M}(s)\right.$, $1 / n)$ ) für die $s \in \Delta_{1}\left(D_{n}\right)$ ist. Es ist offenbar $D_{n+1} \subset D_{n}$. Es sei $\hat{p}^{\prime}$ ein Punkt aus $\hat{M}(s)$ und $G^{\prime}\left(\hat{p}^{\prime}, \varepsilon\right)$ die Menge der Punkte von $R^{\prime}$, deren Entfernung von $\hat{p}^{\prime}$ nicht grösser als $\varepsilon$ ist. Da aber $s \in \Delta_{1}\left(D_{n}\right)$ ist, so haben wir für jedes $n$, $D_{n} \oplus f^{-1}\left(R^{\prime}-\overline{G^{\prime}\left(\hat{p}^{\prime}, \varepsilon\right)}\right)$. Es ist also $D_{n} \cap f^{-1}\left(\overline{G^{\prime}\left(\hat{p}^{\prime}, \varepsilon\right)}\right) \neq \phi$. Es sei jetzt $\left\{\hat{p}_{m}^{\prime}\right\}$ eine dichte Folge in $\hat{M}(s)$ und $p(t)(0 \leq t \leq 1-1 / 2)$ ein Weg auf $D_{1}$, der mit $f^{-1}\left(G^{\prime}\left(p_{1}^{\prime}, \varepsilon\right)\right)$ wenigstens einen Punkt gemeinsam hat und $p(1-1 / 2) \in D_{2}$ ist. Wir nehmen an, dass der Weg $p(t)$ schon für $0 \leq t \leq 1-1 / 2^{n-1}$ konstruiert wurde und $p\left(1-1 / 2^{n-1}\right) \in D_{n}$ ist. Dann konstruieren wir $p(t)$ für $1-1 / 2^{n-1}$ $\leq t \leq 1-1 / 2^{n}$ so, dass er in $D_{n}$ enthalten ist, mit $f^{-1}\left(G^{\prime}\left(\hat{p}_{i}^{\prime}, 1 / 2^{n}\right)\right)(i=1,2$, $\ldots, n)$ wenigstens einen gemeinsamen Punkt hat und $p\left(1-1 / 2^{n}\right) \in D_{n+1}$ ist. Der so konstruierte Weg $\lambda$ besitzt offenbar die oben angegebenen Eigenschaften.

Es sei $\mathfrak{F}(f)$ die Menge der Punkte s aus $\Delta_{1}$ für die $\hat{M}(s)$ aus einem einzigen Punkt besteht. Wir setzen für $s \in \mathfrak{F}(f)$

$$
\hat{f}(s)=\hat{M}(s) .
$$

Die Abbildung $\hat{f}: \mathfrak{F}(f) \rightarrow \hat{R}^{\prime}$ besitzt folgende Eigenschaften :

a) Es ist für jedes $\hat{p} \in \hat{R}^{\prime}$

$$
\hat{f}^{-1}\left(\hat{p}^{\prime}\right)=\bigcap_{n=1}^{\infty} \Delta_{1}\left(f^{-1}\left(G^{\prime}\left(\hat{p}^{\prime}, 1 / n\right)\right)\right) .
$$

b) $\hat{f}$ ist messbar, d.h. $\hat{f}^{-1}\left(\hat{A}^{\prime}\right)$ ist eine Borelsche Menge, für jede Borelsche Menge $\hat{A}^{\prime} \subset \hat{R}^{\prime}$. Insbesondere ist $\mathfrak{F}(f)$ eine Borelsche Menge.

c) $\hat{f}^{-1}\left(\Delta^{\prime}\right) \subset \Delta_{1}(f) ; \quad \hat{f}^{-1}\left(R^{\prime}\right) \subset \Delta_{1}-\Delta_{1}(f)$.

d) Für $s \in \mathfrak{F}(f)$ ist $\hat{f}(s)$ ein asymptotischer Punkt von $f$ in $s$.

e) Es sei $s \in \mathfrak{F}(f)$ und $F$ eine abgeschlossene Menge auf $R$, für die $s \notin \Delta_{1}(R-F)$ ist. Dann gibt es eine Folge $\left\{p_{n}\right\}$ aus $F$, die gegen $s$ strebt, so dass $\lim _{n \rightarrow \infty} K_{s}\left(p_{n}\right)=\sup K_{s}$ ist und die Folge $\left\{f\left(p_{n}\right)\right\}$ gegen $\hat{f}(s)$ konvergiert.

f) Für $R^{\prime} \notin O_{G}, \quad s \in \Delta_{1}$ und $E K_{s} \neq \infty$ ist $s \in \mathfrak{F}(f)$ und $E K_{s}=\alpha K_{\hat{f}(s)}^{\prime}$ mit $\alpha$ eine positive Zahl.

a) Ist $s \in \hat{f}^{-1}\left(\hat{p}^{\prime}\right)$, so gilt nach der Eigenschaft a $)$ von $\hat{M}(s), s \in \Delta_{1}\left(f^{-1}\left(G^{\prime}(\hat{p}\right.\right.$, $1 / n))$ ) für jedes $n$. Ist, umgekehrt, $s \in \Delta_{1}\left(f^{-1}\left(G^{\prime}\left(\hat{p}^{\prime}, 1 / n\right)\right)\right)$ für jedes $n$, so haben wir 


$$
\hat{M}(s) \subset \overline{f\left(f^{-1}\left(G^{\prime}\left(\hat{p}^{\prime}, 1 / n\right)\right)\right.} \subset \overline{G^{\prime}\left(\hat{p}^{\prime}, 1 / n\right)}
$$

und somit $\hat{M}(s)=\left\{\hat{p}^{\prime}\right\}$. Daraus folgt $s \in \mathfrak{F}(f), s \in \hat{f}^{-1}\left(\hat{p}^{\prime}\right)$.

b) Es genügt zu zeigen, dass $\hat{f}^{-1}\left(\hat{A}^{\prime}\right)$ eine Borelsche Menge ist, wenn $\hat{A}^{\prime}$ abgeschlossen ist. Es sei $\left\{\hat{p}_{n}^{\prime}\right\}$ eine dichte Folge auf $\hat{A}^{\prime}$. Es ist offenbar

$$
\hat{f}^{-1}\left(\hat{A}^{\prime}\right) \subset \bigcap_{m=1}^{\infty} \bigcup_{n=1}^{\infty} A_{1}\left(f^{-1}\left(G^{\prime}\left(\hat{p}_{n}^{\prime}, 1 / m\right)\right)\right) \text {. }
$$

Es sei $s \in \bigcap_{m=1}^{\infty} \bigcup_{n=1}^{\infty} \Delta_{1}\left(f^{-1}\left(G^{\prime}\left(\hat{p}_{n}^{\prime}, 1 / m\right)\right)\right)$. Dann gehört der Punkt $s$, für jedes $m$, einer Menge $\Delta_{1}\left(f^{-1}\left(G^{\prime}\left(\hat{p}_{n(m)}^{\prime}, 1 / m\right)\right)\right)$ an. Es sei $\hat{p}^{\prime}$ ein Häufungspunkt der Folge $\left\{\hat{p}_{n(m)}^{\prime}\right\}_{m}\left(\hat{p}^{\prime} \in \hat{A}^{\prime}\right)$ und $\hat{G}^{\prime}$ eine Umgebung von $\hat{p}^{\prime}$. Man kann ein $m$ finden, derart dass $G^{\prime}\left(\hat{p}_{n(m)}^{\prime}, 1 / m\right) \subset \hat{G}^{\prime}$ ist; daraus folgt $s \in \Delta_{1}\left(f^{-1}\left(\hat{G} \cap R^{\prime}\right)\right)$ und $\hat{M}(s) \subset \overline{\hat{G}^{\prime}}$. Da $\hat{G}^{\prime}$ beliebig war, so ersieht man hieraus, dass $\hat{M}(\mathrm{~s})=\left\{\hat{p}^{\prime}\right\}$ ist und $s \in \mathfrak{F}(f), s \in \hat{f}^{-1}\left(\hat{p}^{\prime}\right) \subset \hat{f}^{-1}\left(\hat{A}^{\prime}\right)$ gilt. $\quad \mathrm{Da} \Delta_{1}\left(f^{-1}\left(G^{\prime}\left(\hat{p}_{n}^{\prime}, 1 / m\right)\right)\right)$ Borelsche Mengen sind, so ergibt sich, dass auch $\hat{f}^{-1}\left(\hat{A}^{\prime}\right)$ eine Borelsche Menge und $\hat{f}$ messbar ist.

c) und d) folgen aus den entsprechenden Eigenschaften von $\hat{M}(s)$.

e) Aus $s \in \Delta_{1}(G(s, 1 / n)), s \in A_{1}\left(G_{\alpha_{n}}\right)\left(\alpha_{n} \uparrow \sup K_{s}, G_{\alpha_{n}}=\left\{p \in R \mid K_{s}(p)>\alpha_{n}\right\}\right)$, $s \in \Delta_{1}\left(f^{-1}\left(G^{\prime}(\hat{f}(s), 1 / n)\right)\right)$ und Hilfssatz 10 folgt

$$
s \in \Delta_{1}\left(G(s, 1 / n) \cap G_{\alpha_{n}} \cap f^{-1}\left(G^{\prime}(\hat{f}(s), 1 / n)\right)\right) .
$$

Da $s \notin \Delta_{1}(R-F)$ ist, so ist die Menge $G(s, 1 / n) \cap G_{\alpha_{n}} \cap f^{-1}\left(G^{\prime}(\hat{f}(s), 1 / n)\right)$ nicht in $R-F$ enthalten. Es gibt also einen Punkt $p_{n}$,

$$
p_{n} \in G(s, 1 / n) \cap G_{\alpha_{n}} \cap f^{-1}\left(G^{\prime}(\hat{f}(s), 1 / n)\right) \cap F .
$$

Die Folge $\left\{p_{n}\right\}$ besitzt offenbar die gesuchte Eigenschaft.

f) Nach Satz 7 ist $E K_{s}=\alpha g_{p^{\prime}}^{\prime}\left(\not^{\prime} \in R^{\prime}\right)$, oder $E K_{s}=\alpha K_{s^{\prime}}^{\prime}\left(s^{\prime} \in \Delta_{1}^{\prime}\right)$. Im ersten Fall sei $G^{\prime}$ eine relativkompakte Umgebung von $p^{\prime}$. Auf dem Rand von $f^{-1}\left(G^{\prime}\right)$ ist $K_{s}$ nicht grösser als $\alpha g_{p^{\prime}}^{\prime} \circ f$ und somit beschränkt. Nach Hilfssatz 4 ist

$$
K_{s}=\underset{f^{-1}\left(\sigma^{\prime}\right)}{I} K_{s}+H_{f^{-1}\left(G^{\prime}\right)}^{K_{S}}
$$

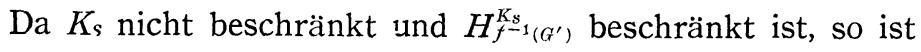

$$
\underset{f^{-1}\left(G^{\prime}\right)}{I} K_{s} \neq 0 .
$$

Es ist alșo $s \in A_{1}\left(f^{-1}\left(G^{\prime}\right)\right), s \in \widetilde{F}(f)$ und $\hat{f}(s)=p^{\prime}$. Im zweiten Fall sei $\hat{G}^{\prime}$ eine 
Umgebung von $s^{\prime}$ und $G^{\prime}=\hat{G}^{\prime} \cap R^{\prime}$. Da

$$
\underset{G^{\prime}}{I} K_{S^{\prime}}^{\prime} \neq 0, \quad I_{f} K_{S^{\prime}}^{\prime} \geq \frac{1}{\alpha} K_{s} \neq 0
$$

ist, so folgt aus dem Hilfssatz 6,

$$
\underset{f^{-1}\left(G^{\prime}\right)}{E} \underset{f^{-1}\left(G^{\prime}\right)}{I} I_{f} K_{s^{\prime}}^{\prime}=I_{f} K_{s^{\prime}}^{\prime}
$$

Nach Hilfssatz 3 ist

$$
\underset{f^{-1}\left(G^{\prime}\right)}{E} \underset{f^{-1}\left(G^{\prime}\right)}{I} K_{\varsigma}=K_{\varsigma}
$$

und $s \in \Delta_{1}\left(f^{-1}\left(G^{\prime}\right)\right)$. Es ist also $s \in \mathfrak{F}(f)$ und $\hat{f}(s)=s^{\prime}$.

SAtz $16^{13)}$ (Riesz-Lusin-Privaloff-Frostman-Nevanlinna). Es sei $\hat{A}^{\prime}$ eine polare Menge auf $\hat{R}^{\prime}$. Ist für eine Menge $A \subset \Delta_{1}, \hat{M}(s) \subset \hat{A}^{\prime}$ für jedes $s \in A$, so ist $A$ vom harmonischen Masse Null. insbesondere ist $\hat{f}^{-1}\left(\hat{A}^{\prime}\right)$ eine Menge vom harmonischen Masse Null.

Es sei $G^{\prime}$ eine Kreisscheibe auf $R^{\prime}$ und $S^{\prime} \in S P\left(R^{\prime}-\bar{G}^{\prime}\right), S^{\prime} \neq \infty$ und

$$
\lim _{p^{\prime} \rightarrow \hat{q}^{\prime} \in \hat{A}^{\prime}-G^{\prime}} S^{\prime}\left(p^{\prime}\right)=\infty
$$

Wir bezeichnen

$$
\begin{aligned}
& A_{a^{\prime}}=\left\{s \in A \mid \hat{M}(s) \subset \hat{A}^{\prime}-\bar{G}^{\prime}\right\}, \\
& G_{\alpha}^{\prime}=\left\{p^{\prime} \in R^{\prime}-\bar{G}^{\prime} \mid S^{\prime}\left(p^{\prime}\right)>\alpha\right\}, \\
& D=f^{-1}\left(R^{\prime}-\bar{G}^{\prime}\right), G_{\alpha}=f^{-1}\left(G_{\alpha}^{\prime}\right) \subset D,
\end{aligned}
$$

$\eta_{\alpha}$ die identische Abbildung von $G_{\alpha}$ in $D$ und $\eta$ die identische Abbildung von $D$ in $R$. Es sei $s \in A_{G^{\prime}}$. Dann ist $G^{\prime}(\hat{M}(s), \varepsilon) \subset G_{\alpha}^{\prime}$ für ein $\varepsilon>0$ und $s \in \Delta_{1}\left(f^{-1}\left(G^{\prime}(\hat{M}(s), \varepsilon)\right)\right) \subset \Delta_{1}\left(G_{\alpha}\right), A_{G^{\prime}} \subset \Delta_{1}\left(G_{\alpha}\right)$. Wir werden beweisen, dass

$$
\lim _{\alpha \rightarrow \infty} \omega\left(\Delta_{1}\left(G_{\alpha}\right), R\right)=0
$$

und somit $A_{\sigma^{\prime}}$ vom harmonischen Masse Null ist. Auf $G_{\alpha}$ ist

$$
I_{\eta \circ \eta_{\alpha}} 1 \leq 1 \leq \frac{1}{\alpha} S^{\prime} \circ f
$$

Daraus folgt

$$
E_{\eta_{\alpha}} I_{\eta_{\circ} \eta_{\alpha}} 1 \leq \frac{1}{\alpha} S^{\prime} \circ f
$$

13) Siehe auch Theorem 6-2 von [6] im Falle, dass $f$ eine Lindelofsche Abbildung ist. 
auf $D$ und

$$
\lim _{\alpha \rightarrow \infty} E_{\eta_{\alpha}} I_{\eta_{\circ} \eta_{\alpha}} 1=0
$$

Aus

$$
E_{\eta_{\alpha}} I_{\eta_{\circ} \eta_{\alpha}} 1=E_{\eta_{\alpha} I_{\eta_{\alpha}}} I_{\eta} 1 \leq I_{\eta} 1
$$

folgt $E_{\eta_{\alpha}} I_{\eta_{\circ \eta_{\alpha}}} 1 \in H P(\eta)$. Die Folge $\left\{E_{\eta_{n}} I_{\eta_{0} \circ \eta_{n}} 1\right\}_{n}$ ist eine nichtzunehmende Folge aus $H P(\eta)$ und nach Satz 6 ist

$$
\lim _{n \rightarrow \infty} E_{\eta} E_{\eta_{n}} I_{\eta_{0} \eta_{n}} 1=0 .
$$

Nach Satz $15^{\prime}$ ist aber

$$
E_{\eta} E_{\eta_{n}} I_{\eta_{\circ} \eta_{n}} 1=E_{\eta_{\circ} \eta_{n}} I_{\eta \circ \eta_{n}} 1=\omega\left(\Delta_{1}\left(G_{n}\right), R\right),
$$

woraus

$$
\lim _{n \rightarrow \infty} \omega\left(\Delta_{1}\left(G_{n}\right), R\right)=0
$$

folgt. Es sei $s \in A-A_{G^{\prime}}$. Dann ist $\hat{M}(s) \cap \bar{G}^{\prime} \cap \hat{A}^{\prime} \neq \phi . \quad$ Da aber $\hat{A}^{\prime} \cap \bar{G}^{\prime}$ eine Menge der Kapazität Null und $\hat{M}(s)$ ein Kontinuum ist, so reduziert sich $\hat{M}(s)$ auf einen Punkt aus $\bar{G}^{\prime}$. Ist $\Omega^{\prime}$ eine zweite Kreisscheibe auf $R^{\prime}$, so dass $\bar{\Omega}^{\prime} \cap \overline{G^{\prime}}=\phi$ ist, so ist $s \in A_{\Omega^{\prime}}$ und $A \subset A_{G^{\prime}} \cup A_{\Omega^{\prime}}$. Daraus folgt, dass $A$ eine Menge vom harmonischen Masse Null ist.

Hilfssatz 12. Hat $R^{\prime}$ eine Greensche Funktion, so ist die Menge $\hat{f}^{-1}\left(s^{\prime}\right)$ für jeden Punkt $s^{\prime} \in \Delta_{1}^{\prime}(f)$ nicht leer und

$$
I K_{s^{\prime}}^{\prime}=\int_{\hat{f}^{-1}\left(s^{\prime}\right)} K_{s} d\left(\delta_{i^{\prime}} \circ f\right)(s) .
$$

Da

$$
I_{f} K_{s^{\prime}}^{\prime} \neq 0, \quad \underset{\sigma^{\prime}\left(s^{\prime}, \varepsilon\right)}{I} K_{s^{\prime}}^{\prime} \neq 0
$$

ist, so folgt aus dem Hilfssatz 6,

$$
\underset{f^{-1}\left(G^{\prime}\left(S^{\prime}, \varepsilon\right)\right)}{E} \underset{f^{-1}\left(G^{\prime}\left(s^{\prime}, \varepsilon\right)\right)}{I} L_{f} K_{s^{\prime}}^{\prime}=I_{f} K_{S^{\prime}}^{\prime}
$$

Nach Satz 14 ist

$$
I_{f} K_{s^{\prime}}^{\prime}=\int_{\Delta_{1}(f)} K_{s} d\left(\delta_{s^{\prime}} \circ f\right)(s)
$$

Aus diesen Gleichheiten und aus Satz $15^{\prime}$ ergibt sich

$$
I_{f} K_{s^{\prime}}^{\prime}=\int_{\Delta_{1}(f) \cap \Delta_{1}\left(f^{-1}\left(G^{\prime}\left(s^{\prime}, \varepsilon\right)\right)\right)} K_{s} d\left(\delta_{s^{\prime}} \circ f\right)(s) .
$$


Aus den Eigenschaften a) und c) von $\hat{f}$ erhalten wir

$$
\begin{gathered}
I_{f} K_{s^{\prime}}^{\prime}=\lim _{n \rightarrow \infty} \int_{\Delta_{1}(f) \cap \Delta_{1}\left(f^{-1}\left(G^{\prime}\left(s^{\prime}, 1 / n\right)\right)\right)} K_{s} d\left(\delta_{s^{\prime}} \circ f\right)(s)=\int_{\Delta_{1}(f) \cap \hat{f}^{-1}\left(s^{\prime}\right)} K_{s} d\left(\delta_{s}, \circ f\right)(s) \\
=\int_{\hat{f}^{-1}\left(s^{\prime}\right)} K_{s} d\left(\delta_{s^{\prime}} \circ f\right)(s) .
\end{gathered}
$$

SATZ 17. Ist $R^{\prime} \notin O_{G}$ und

$$
u^{\prime}=\int_{A^{\prime}} K_{s^{\prime}}^{\prime} d \mu^{\prime}\left(s^{\prime}\right) \in H P\left(R^{\prime}\right),
$$

wo $A^{\prime}$ eine Borelsche Menge auf $\Delta_{1}^{\prime}$ ist, so ist

$$
I u^{\prime}=\int_{\hat{f}^{-1}\left(A^{\prime}\right)} K_{s} d\left(\mu^{\prime} \circ f\right)(s)=\int_{\widetilde{F}(f) \cap \Delta_{1}(f)} K_{s} d\left(\mu^{\prime} \circ f\right)(s)=\int_{\Delta_{1}(f)} K_{s} d\left(\mu^{\prime} \circ f\right)(s) .
$$

Es ist

$$
\begin{aligned}
& I \omega\left(A^{\prime}, R^{\prime}\right)=\omega\left(\hat{f}^{-1}\left(A^{\prime}\right), R\right), \\
& \chi_{R}\left(\Delta_{1}(f)-\tilde{f}(f)\right)=0 .
\end{aligned}
$$

Nach den Eigenschaften b), c) von $\hat{f}$, Satz 14 und Hilfssatz 12 ist

$$
I K_{s^{\prime}}^{\prime}=\int_{\hat{f}^{-1}\left(s^{\prime}\right)} K_{s} d\left(\delta_{s^{\prime}} \circ f\right)(s)=\int_{\hat{f}^{-1}\left(A^{\prime}\right)} K_{s} d\left(\delta_{s^{\prime}} \circ f\right)(s)
$$

für jedes $s^{\prime} \in A^{\prime}$. Es ist also nach Satz 14

$$
I u^{\prime}=\int_{\hat{f}^{-1}\left(A^{\prime}\right)} K_{s} d\left(\mu^{\prime} \circ f\right)(s) .
$$

Daraus und aus dem Satz 14 folgen sofort die anderen Gleichheiten aus der ersten Reihe. Die letzten Behauptungen des Satzes ergeben sich aus diesen Gleichheiten durch Betrachtung der Beziehungen

$$
\omega\left(A^{\prime}, R^{\prime}\right)=\int_{A^{\prime}} K_{S^{\prime}}^{\prime} d \chi_{P^{\prime}}\left(s^{\prime}\right), \quad \chi_{R^{\prime}} \circ f=\chi_{R}
$$

Hilfssatz 13. Es sei F eine abgeschlossene Menge der Kapazität Null auf $R, \stackrel{R}{R}=R-F, \eta$ die identische Abbildung von $\hat{R}$ in $R$, und $\dot{A}$ der ideale Rand von $\stackrel{R}{R}$. Dann ist $\hat{\eta}$ überall auf $\dot{J}_{1}(\eta)$ definiert; $\hat{\eta}$ bildet $\stackrel{\Delta}{1}_{1}(\eta)$ topologisch auf $\Delta_{1}$ und

$$
\hat{M}_{f}(s)=\hat{M}_{f \circ \eta}\left(\hat{\eta}^{-1}(s)\right)
$$

für jedes $s \in \Delta_{1}$. Ist $\AA \subset \bigsqcup_{1}(\eta)$ eine Borelsche Menge vom harmonischen Masse 
Null, so ist auch $\hat{\eta}(\AA)$ eine Borelsche Menge vom harmonischen Masse Null.

Wir konstruieren den idealen Rand von $R$ in Bezug auf einen Punkt $p_{0} \in R-F$ und den idealen Rand von $\hat{R}$ in Bezug auf $\eta^{-1}\left(p_{0}\right)$. Es sei $\stackrel{s}{s} \in \stackrel{\circ}{\Delta}_{1}(\eta)$

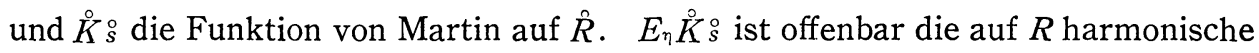
Funktion, die in allen Punkten von $R-F$ gleich $\left(\stackrel{\circ}{K}_{s}^{\circ}\right) \circ \eta^{-1}$ ist. Nach der

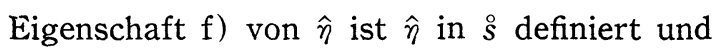

$$
E_{\eta} \stackrel{\circ}{K} \stackrel{\circ}{s}=K \hat{\eta}(\stackrel{\circ}{s}) \text {. }
$$

Aus $\hat{\eta}\left(\stackrel{\circ}{s}_{1}\right)=\hat{\eta}\left(\stackrel{\circ}{s}_{2}\right)$ folgt, dass die Funktionen $\stackrel{\circ}{K}_{s}^{\circ}, \quad \stackrel{\circ}{K}_{s_{2}}^{\circ}$ gleich sind und somit die Punkte $\stackrel{\circ}{1}_{1}, \stackrel{\circ}{s}_{2}$ zusammenfallen; $\hat{\eta}$ ist also eineindeutig. Es sei $s \in \Delta_{1}$. Dann ist offenbar $K_{s}{ }^{\circ} \eta$ eine minimale Funktion auf $\hat{R}$ aus $H P(\eta)$. Daraus folgt, dass ein $\stackrel{\circ}{s} \in \stackrel{\triangle}{1}_{1}(\eta)$ existiert, so dass $K_{s} \circ \eta=\stackrel{\circ}{K}_{s}^{\circ}$ ist. Es ist also $s=\hat{\eta}\left(s_{0}\right)$ und $\hat{\eta}$ ist auf $\Delta_{1}$. Aus

$$
\left.K \hat{\eta}(\stackrel{\circ}{s})=(\stackrel{\circ}{K} \stackrel{\circ}{s}) \circ \eta^{-1}, \quad K_{s} \circ \eta=\stackrel{\circ}{K} \hat{\eta}-1, s\right)
$$

sieht man, dass $\hat{\eta}$ und $\hat{\eta}^{-1}$ stetig sind. Es sei $G$ eine offene Menge auf $R$. Ist $s \in \Delta_{1}(G)$, so ist $\hat{\eta}^{-1}(s) \in \stackrel{\Delta}{1}_{1}\left(\eta^{-1}(G)\right)$ und umgekehrt. Daraus und aus $\overline{f(G)}=\overline{f \circ \eta\left(\eta^{-1}(G)\right)}$ folgt sofort

$$
\hat{M}_{f}(s)=\hat{M}_{f \circ \eta}\left(\hat{\eta}^{-1}(s)\right) .
$$

Die letzte Behauptung ergibt sich aus

$$
\omega(\hat{\eta}(\AA), R) \circ \eta=I_{\eta} \omega(\hat{\eta}(\AA), R)=\omega(\AA, \AA R)=0 .
$$

SATz 18 (Fatou-Nevanlinna). Ist $f$ eine Lindelöfsche Abbildung [6], (was imrrer der Fall ist, wenn $R^{\prime} \notin O_{G}$ ist), so ist $\hat{f}$ fast überall auf $\Delta_{1}$ definiert, d.h.

$$
\chi_{R}\left(\Delta_{1}-\widetilde{F}(f)\right)=0
$$

Wir bezeichnen mit $\underline{\Delta_{1}(f)}$ die Menge der Punkte $s \in \Delta_{1}$ für die $\hat{M}(s) \subset \Delta^{\prime}$

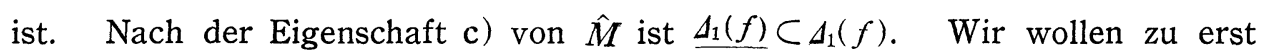
beweisen, dass

$$
\chi_{R}\left(\Delta_{1}-\underline{\Delta_{1}(f)}-\mathfrak{F}(f)\right)=0
$$

ist. Für zwei beliebige Punkte $p^{\prime}, q^{\prime}$ aus $R^{\prime}$ gibt es eine Funktion $v_{p^{\prime} q^{\prime}}$ auf $R^{\prime}$ die ausserhalb der Punkte $p^{\prime}, q^{\prime}$ harmonisch ist, im Punkte $p^{\prime}$, bzw. $q^{\prime}$, eine positive, bzw. negative, logarithmische Singularität besitzt und auf dem idealen Rand normiert ist, d.h. es ist 


$$
H_{R^{\prime}-\bar{G}^{\prime}}^{v p^{\prime} q^{\prime}}=v_{p^{\prime} q^{\prime}}
$$

für ein relativkompaktes Gebiet $G^{\prime}$, das die Punkte $p^{\prime}, q^{\prime}$ enthält. Wir bezeichnen

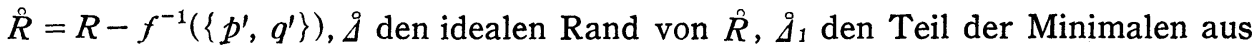
$\stackrel{\AA}{\Delta, \eta}$ die identische Abbildung von $\hat{R}$ in $R$ und

$$
\begin{aligned}
& G_{\alpha}^{\prime}=\left\{r^{\prime} \in R^{\prime} \mid v_{p^{\prime} q^{\prime}}\left(r^{\prime}\right)>\alpha\right\}, \\
& \stackrel{\circ}{\alpha}_{\alpha}=(f \circ \eta)^{-1}\left(G_{\alpha}^{\prime}\right) .
\end{aligned}
$$

Die Behauptung, dass $f$ eine Lindelöfsche Abbildung ist, ist mit der Behauptung äquivalent, dass die Funktion $v_{p^{\prime} q^{\prime}} \circ f \circ \eta$ eine Differenz von zwei nichtnegativen harmonischen Funktionen ist [6]. Nach dem Hilfssatz 11 gibt es eine dichte abzählbare Menge von reellen Zahlen $\mathfrak{N}_{p^{\prime} q^{\prime}}$ derart, dass für jedes $\alpha \in \mathfrak{N}_{p^{\prime} q^{\prime}}$

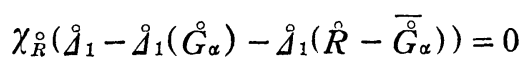

ist. Wir setzen weiter

$$
\begin{aligned}
& \check{Z}_{p^{\prime} q^{\prime}}=\bigcup_{\alpha \in \Re p^{\prime} q^{\prime}}\left(\stackrel{\leftrightarrow}{1}_{1}-\AA_{1}\left(\stackrel{\circ}{G}_{\alpha}\right)-\AA_{1}\left(\hat{R}-\overline{\mathscr{G}}_{\alpha}\right)\right), \\
& Z_{p^{\prime} q^{\prime}}=\hat{\eta}\left({\stackrel{Z}{p^{\prime} q^{\prime}}}\right) \text {. }
\end{aligned}
$$

Nach Hilfssatz 13 ist $\chi_{R}\left(Z_{p^{\prime} q^{\prime}}\right)=0$. Es sei $s \in \Delta_{1}-\underline{\Delta_{1}(f)}-Z_{p^{\prime} q^{\prime}}$ und $\stackrel{\circ}{s}=\hat{\eta}^{-1}(s)$ $\in \mathscr{S}_{1}(\eta)-\grave{Z}_{p^{\prime} q^{\prime}}$. Für jedes $\alpha \in \Re_{p^{\prime} q^{\prime}}$ ist entweder $\hat{s} \in \mathscr{J}_{1}\left(\hat{G}_{\alpha}\right)$ oder $\stackrel{o}{s} \in \stackrel{\triangle}{1}_{1}\left(\hat{R}-\overrightarrow{\hat{G}}_{\alpha}\right)$, und somit ist entweder

$$
\hat{M}_{f}(s)=\hat{M}_{f \circ \eta}\left(\stackrel{\diamond}{s} \subset \bar{G}_{\alpha}^{\prime}\right.
$$

oder

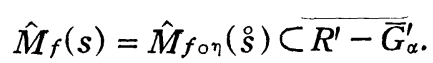

Es sei

$$
\alpha_{0}=\sup \left\{v_{p^{\prime} q^{\prime}}\left(r^{\prime}\right) \mid r^{\prime} \in \hat{M}_{f}(s) \cap R^{\prime}\right\}^{14)}
$$

Für jedes $\alpha \in \mathfrak{N}_{p^{\prime} q^{\prime}}\left(\alpha<\alpha_{0}\right)$, ist $\hat{M}_{f}(s) \notin \overline{R^{\prime}-\bar{G}_{\alpha}^{\prime}}$ und somit ist

$$
\hat{M}_{f}(s) \subset \bar{G}_{\alpha}^{\prime} .
$$

Daraus folgt, da $\Re_{p^{\prime} q^{\prime}}$ dicht ist, $\hat{M}_{f}(s) \subset \bar{G}_{\alpha_{0}}^{\prime}$ und wir haben

$$
\hat{M}_{f}(s) \subset\left\{r^{\prime} \in R^{\prime} \mid v_{p^{\prime} q^{\prime}}\left(r^{\prime}\right)=\alpha_{0}\right\} .
$$

Für fast alle Punkte von $\Delta_{1}-\underline{\Delta_{1}(f)}$ ist $\hat{M}_{f}(s)$ in den Niveaukurven von $v_{p \cdot q}$

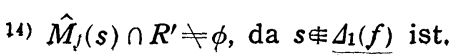


enthalten.

Es sei $\left\{p_{i}^{\prime}\right\}$ eine dichte Folge auf $R^{\prime}$ und

$$
Z=\bigcup_{i=1}^{\infty} Z_{p_{i^{\prime} q^{\prime}}}
$$

es ist $\chi_{R}(Z)=0$ und für jedes $s \in \Delta_{1}-\underline{\Delta_{1}(f)}-Z$ ist $\hat{M}_{f}(s)$ in den Niveaukurven aller Funktionen $v_{p_{i^{\prime}} q^{\prime}}$ enthalten, d.h. $\hat{M}_{f}(s)$ reduziert sich auf einen Punkt, da noch Eigenschaft b) von $\hat{M}, \hat{M}_{f}(s)$ zusammenhängend ist. Es ist also $s \in \mathfrak{F}(f)$,

$$
\Delta_{1}-\underline{\Delta_{1}(f)}-\mathfrak{F}(f) \subset Z,
$$

und

$$
\chi_{R}\left(\Delta_{1}-\underline{\Delta_{1}(f)}-\mathfrak{F}(f)\right)=0 .
$$

Ist $R^{\prime}$ kompakt, so ist $\Delta_{1}(f)$ leer, und $\operatorname{der}$ Satz ist bewiesen. Ist $\mathrm{R}^{\prime}$ offen und $R^{\prime} \in O_{G}$, so ist nach Satz 16

$$
\chi_{R}\left(\underline{A_{1}(f)}\right)=0,
$$

da $\Delta^{\prime}$ eine polare Menge ist und somit haben wir $\chi_{R}\left(\Delta_{1}-\mathfrak{F}(f)\right)=0$. Ist $R^{\prime} \notin O_{G}$, so ist nach Satz 17

$$
\chi_{R}\left(\Delta_{1}(f)-\mathfrak{F}(f)\right)=0 .
$$

Daraus und aus $\underline{\Delta_{1}(f)} \subset \Delta_{1}(f)$ schliessen wir

$$
\chi_{R}\left(\Delta_{1}-\mathfrak{F}(f)\right) \leq \chi_{R}\left(\Delta_{1}-\underline{\Delta}_{1}(f)-\mathfrak{F}(f)\right)+\chi_{R}\left(\Delta_{1}(f)-\mathfrak{F}(f)\right)=0,
$$

womit der Satz vollständig bewiesen ist.

Folgesatz 3. Es sei $R \in U$ und $K_{\varsigma}$ eine beschränkte Minimale. Dann entstehen folgende zwei Möglichkeiten: entweder besitzt $R^{\prime}$ eine Greensche Funktion, und dann ist $\hat{f}$ in s definiert, $\hat{f}(s) \in \Delta_{1}^{\prime}, K_{\hat{f}(s)}^{\prime}$ ist eine beschränkte Minimale und $R^{\prime} \in U$, oder aber besitzt $R^{\prime}$ keine Greensche Funktion, und dann ist $\hat{f}$ in $s$ nicht definiert und $f$ ist keine Lindelöfsche Abbildung.

$\{s\}$ ist eine Menge vom positiven harmonischen Masse. Nach Satz 18 ist also $\hat{f}$ in $s$ definiert, falls $R^{\prime} \notin O_{G}$. Ist, umgekehrt, $\hat{f}$ in $s$ definiert, so ist $\hat{f}(s)$ nach Satz 16 keine polare Menge. Das kann nur dann der Fall sein, wenn $R^{\prime} \notin O_{G}$ und $K_{\hat{f}(s)}^{\prime}$ eine beschränkte Minimale ist.

Folgesatz 4 [1]. Jede Fortsetzung einer Riemannschen Fläche der Klasse U gehört der Klasse $U$ an. 
Es sei $R \in U$ und $R^{\prime} \supset R$. Die identische Abbildung ist offenbar eine Lindelöfsche Abbildung. Aus dem Folgesatz 3 schliesst man zuerst $R^{\prime} \notin O_{G}$ und dann $R^{\prime} \in U$.

Heins hat mit $O_{L}$ die Klasse der Riemannschen Flächen mit Greenscher Funktionen bezeichnet, die keine Lindelöfsche Abbildung in der Riemannschen Kugel $(|w| \leq \infty)$ besitzen [6]. Es sei $O_{L^{\prime}}$ die Klasse der Riemanschen Flächen mit Greenschen Funktionen, die keine Lindelöfsche Abbildungen in Riemannschen Fläche aus der Klasse $O_{G}$ besitzen. Es ist offenbar

$$
O_{L}, \subset O_{L}
$$

Aus dem Folgesatz 3 ergibt sich

$$
U \subset O_{L^{\prime}}
$$

Man kann ein von Heins [4] gegebenes Beispiel benutzen, um zu beweisen, dass die obige Inklusion $O_{L^{\prime}} \subset O_{L}$ echt ist.

SAtz 19 (Löwner) Es sei $R^{\prime} \notin O_{G}, A$ eine Borelsche Menge auf $\Delta$ und $A^{\prime}$ eine Borelsche Menge auf $\Delta^{\prime}$. Sind alle Limes von $f$ in $A$ in $A^{\prime}$ enthalten, so ist

$$
\omega(A, R) \leq \omega\left(A^{\prime}, R^{\prime}\right) \circ f .
$$

Ist $\hat{f}$ auf $A$ definiert, und ist $\hat{f}(A) \subset \Delta^{\prime}$, so ist offenbar $\hat{f}(A)$ eine analytische $\chi^{\prime}$-messbare Menge und $\omega(A, R) \leqq \omega\left(\hat{f}(A), R^{\prime}\right) \circ f$.

Nach Satz 18 ist

$$
\omega(A, R)=\omega(A \cap \mathfrak{F}(f), R) .
$$

Es sei $s \in A \cap \mathfrak{F}(f)$. Dann ist $\hat{f}(s) \in A^{\prime}$, denn $\hat{f}(s)$ ist nach Eigenschaft d) von $\hat{f}$ ein asymptotischer Punkt in s. Daraus folgt $A \cap \mathfrak{F}(f) \subset \hat{f}^{-1}\left(A^{\prime}\right)$ und

Nach Satz 17 ist

$$
\omega(A \cap \mathfrak{F}(f), R) \leq \omega\left(\hat{f}^{-1}\left(A^{\prime}\right), R\right) .
$$

$$
\omega\left(\hat{f}^{-1}\left(A^{\prime}\right), R\right)=I \omega\left(A^{\prime}, R^{\prime}\right) \leq \omega\left(A^{\prime}, R^{\prime}\right) \circ f,
$$

was $z u$ beweisen war.

$$
\text { V. Der Fall } R=\{|z|<1\}
$$

In diesem Abschnitt werden wir als Riemannsche Fläche $R$ den Kreis $\{|z|<1\}$ nehmen. Wir konstruieren den idealen Rand von Martin in Bezug auf dem Punkt $p_{0}=0 . \quad \hat{R}$ ist homeomorph $z \mathrm{u}\{|z| \leq 1\}, \Delta \mathrm{zu}\{|z|=1\}$ und 
$\Delta_{1}=\Delta$. Wir werden somit als Punkte von $\Delta_{1}$ die Punkte $e^{i \theta}$ nehmen. Es ist

$$
K_{e^{i \theta}}=R e \frac{e^{i \theta}+z}{e^{i \theta}-z} .
$$

$\%_{R}$ ist in diesem Falle gleich dem Lebesgueschen Masse, geteilt durch $2 \pi$. Eine Menge auf $\Delta_{1}$ ist also vom harmonischen Masse Null dann und nur dann, wenn sie vom Lebesgueschen Masse Null ist.

Wir sagen, dass eine Menge $F$ auf $R$ im Punkte $e^{i \theta}$ einen Winkeleingang hat, wenn man mindestens eine Folge $\left\{z_{n}\right\}$ aus $F$ wählen kann, die gegen $e^{i \theta}$ konvergiert und für die

$$
\lim _{n \rightarrow \infty}\left|\arg \frac{e^{i \theta}-z_{n}}{e^{i \theta}}\right|<\frac{\pi}{2}
$$

ist. An Stelle dieser Beziehung werden wir kurz

$$
z_{n} \rightarrow \ngtr e^{i \theta}
$$

schreiben. Eine äquivalente Beziehung, die wir öfter benutzen, ist

$$
\lim _{n \rightarrow \infty} \frac{\left|\theta-\arg z_{n}\right|}{1-\left|z_{n}\right|}<\infty .
$$

Ist $A$ eine Menge auf $|z|=1$, so sagen wir, dass $F$ einen Winkeleingang in $A$ hat, falls sie in jedem Punkt von $A$ einen Winkeleingang hat. Weiter verstehen wir durch den Ausdruck " $f$ hat im Punkte $e^{i \theta}$ den Winkelgrenzpunkt $\hat{p}^{\prime} \in \hat{R}$ " die Beziehung

$$
\lim _{z \rightarrow ð e^{i \theta}} f(z)=\hat{p}^{\prime}
$$

Wir werden $\min \mathfrak{F}^{*}(f)$ die Menge der Punkte $e^{i \theta}$ bezeichnen, für die $f$ einen Winkelgrenzpunkt hat und mit $f^{*}$ die Abbildung $\mathfrak{F}^{*}(f) \rightarrow R^{\prime}$,

$$
f^{*}\left(e^{i \theta}\right)=\lim _{z \rightarrow \chi e^{i \theta}} f(z) .
$$

Es ist bekannt, dass $\mathfrak{F}^{*}(f)$ eine Borelsche Menge und $f^{*}$ eine messbare Abbildung ist.

Satz $20^{15)}$. Die Abbildung $\hat{f}$ ist fast überall auf $\mathfrak{F}^{*}(f)$ definiert und gleich der Abbildung $f^{*}$.

Es sei $A$ die Menge der Punkte $e^{i \theta} \in \mathfrak{F}^{*}(f)$, wo $\hat{M}\left(e^{i \theta}\right) \neq\left\{f^{*}\left(e^{i \theta}\right)\right\}$ ist.

15) Dieser Satz ist auch dann gültig, wenn $R^{\prime}$ nur ein topologischer Raum ist. 
Wir nehmen an, dass $A$ vom positiven Lebesgueschen Masse ist. Es sei

$$
G\left(e^{i \theta}, r\right)=\left\{z|r<| z|<1, \arg | \frac{e^{i \theta}-z}{e^{i \theta}} \mid<\frac{\pi}{4}\right\},
$$

und $A(r, \varepsilon)$ die Menge der Punkte $e^{i \theta} \in A$, für welche $d\left(f^{*}\left(e^{i \theta}\right), f(z)\right)<\varepsilon$ für $z \in G\left(e^{i \theta}, r\right)$ ist. $\mathrm{Da}$

$$
\bigcup_{r<1} A(r, 1 / n)=A
$$

und $A(r, 1 / n)$ zunehmend mit $r$ ist, so kann man ein $r_{n}$ finden, derart dass

$$
\chi\left(A-A\left(r_{n}, \frac{1}{n}\right)\right)<\frac{1}{3^{n}} \chi(A)
$$

gilt. Daraus folgt

$$
\chi\left(\bigcap_{n=1}^{\infty} A\left(r_{n}, \frac{1}{n}\right)\right) \geq \frac{1}{2} \chi(A)>0
$$

Es sei $B \subset \bigcap_{n=1}^{\infty} A\left(r_{n}, 1 / n\right)$ eine perfekte Menge mit positivem Masse und

$$
G=\bigcup_{e^{i} \theta \in B} G\left(e^{i \theta}, \frac{1}{2}\right) \cup\left\{z|| z \mid<\frac{2}{3}\right\}
$$

Ist $\left\{z_{k}\right\}$ eine Folge aus $G$, die gegen $e^{i \theta} \ni B$ konvergiert, so konvergiert $\left\{f\left(z_{k}\right)\right\}$ gegen $f^{*}\left(e^{i \theta}\right)$. In der Tat, für ein beliebiges $\varepsilon>0$ nehmen wir $n$ so gross, dass $1 / n<_{\varepsilon} / 3$ ist. Für $k \geq k_{\varepsilon}$ gibt es ein $\theta_{k}$, so dass $G\left(e^{i \theta}, r_{n}\right) \cap G\left(e^{i \theta_{k}}, r_{n}\right) \neq \phi, e^{i \theta_{k}} \in B$, $z_{k} \in G\left(e^{i \theta_{k}}, 1 / n\right)$ ist. Dann ist für einen Punkt $z_{k}^{\prime} \in G\left(e^{i \theta}, r_{n}\right) \cap G\left(e^{i \theta_{k}}, r_{n}\right)$,

$$
d\left(f^{*}\left(e^{i \theta}\right), f\left(z_{k}\right)\right) \leq d\left(f^{*}\left(e^{i \theta}\right), f\left(z_{k}^{\prime}\right)\right)+d\left(f\left(z_{k}^{\prime}\right), f\left(z_{k}\right)\right)<\frac{\varepsilon}{3}+\frac{2 \varepsilon}{3}=\varepsilon .
$$

Wir wollen jetzt beweisen, dass

$$
\prod_{G} \omega(B, R) \neq 0
$$

ist. Es sei $G_{\alpha}=\{z \in R \mid \omega(z ; B, R)>\alpha\}$; wir werden erst zeigen, dass für $\alpha>1 / 2, G_{\alpha} \subset G$ ist. Es sei $z_{0} \notin G, z_{0}=r e^{i \theta}$. Dann gehört $e^{i \theta}$ der Menge $B$ nicht an; es sei $\lambda$ die Komponente der Komplementarmenge von $B$ in Bezug auf $|z|=1$, die $e^{i \theta}$ enthält; $\lambda$ ist ein Kreisbogen und $\omega(B, R) \leq 1-\omega(\lambda, R)$. Man kann geometrisch zeigen, dass

$$
\omega\left(z_{0} ; \lambda, R\right)>\frac{1}{2}
$$


ist, woraus

$$
\omega\left(z_{0} ; B, R\right)<\frac{1}{2}
$$

und $z_{0} \notin G_{\alpha}$ folgt. $\quad \mathrm{Da} \omega(B, R)-\alpha$ auf $G_{\alpha}$ der Klasse $\mathfrak{U}_{G_{\alpha}}$ angehört und kleiner als $\omega(B, R)$ ist, so ist auf $G_{\alpha}$

$$
0<\omega(B, R)-\alpha \leq I_{G \alpha} \omega(B, R) \leq I_{G} \omega(B, R),
$$

woraus man erkennt, dass

$$
\prod_{G} \omega(B, R) \neq 0
$$

ist. Daraus schliesst man, dass $e^{i \theta} \in \Delta_{1}(G)$ für wenigstens ein $e^{i \theta} \in B$ ist.

Es sei $\hat{G}^{\prime}$ eine Umgebung von $f^{*}\left(e^{i \theta}\right)$. Da $f$ in $e^{i \theta}$ und auf $G$ stetig ist, so kann man eine Umgebung $G_{0}$ von $e^{i \theta}$ finden, so dass $G_{0} \cap G \subset f^{-1}\left(\hat{G}^{\prime} \cap R^{\prime}\right)$ ist. Da $e^{i \theta} \in \Delta_{1}\left(G_{0}\right)$ ist, so ist nach Hilfssatz $10, e^{i \theta} \in \Delta_{1}\left(G_{0} \cap G\right)$ und $\hat{M}\left(e^{i \theta}\right) \subset \overline{G^{\prime}}$. Da $\hat{G}^{\prime}$ eine beliebige Umgebung von $f^{*}\left(e^{i \theta}\right)$ ist, so reduziert sich $\hat{M}\left(e^{i \theta}\right)$ auf diesem Punkt und $e^{i \theta} \in \mathfrak{F}(f)$ entgegen der Vorraussetzung. Es ist somit $A$ vom Lebesgueschen Masse Null, woraus die Behauptungen des Satzes folgen.

Es ist uns nicht gelungen ein Beispiel zu kontruieren in welchem $\mathfrak{\mho}^{*}(f)-$ $\mathfrak{F}(f)$ nicht leer ist.

Es sei $f$ eine analytische Abbildung des Kreises $\{|z|<1\}$ in einer beliebigen Riemannschen Fläche $R^{\prime}$ und $A \subset \mathfrak{F}^{*}(f)$; ist $f^{*}(A)$ eine polare Menge, so ist A vom Lebesgueschen Masse Null (Siehe auch Ohtsuka M., On the boundary volues of analytic transformation of a circle onto a Riemann surface, Nagoya Math. Journ., 10 (1956), pp. 171-175). Ist $R^{\prime}$ die Riemannsche Kugel, so erhalten wir gerade den Satz von Riesz-Lusin-Priwaloff-Frostman-Nevanlinna.

Hilfssatz 14. Es sei $\left\{z_{n}\right\}$ eine Punktfolge aus $|z|<1$ die gegen $z=1$ konvergiert und $\gamma_{n}(n=1,2, \ldots)$ ein Kontinuum, das den Punkt $z_{n}$ enthält und dessen hyperbolischer Durchmesser gleich o (eine fixe von $n$ unabhängige Zahl) ist. Ist

$$
\lim _{n \rightarrow \infty} \frac{\arg z_{n}}{1-\left|z_{n}\right|}=\alpha \quad(-\infty \leq \alpha \leq+\infty)
$$

so ist

$$
\begin{aligned}
& \varlimsup_{n \rightarrow \infty} K_{\gamma_{n}}^{*}(0) \leq \frac{\bar{c}_{\delta}}{1+\alpha^{2}}, \\
& \lim _{n \rightarrow \infty} K_{\gamma_{n}}^{*}(0) \geq \frac{\underline{c_{\delta}}}{1+\alpha^{2}} .
\end{aligned}
$$


Dabei ist $K=K_{1}=\operatorname{Re} \frac{1+z}{1-z}$ und $\bar{c}_{\delta}, \underline{c}_{\delta}$ sind endliche positive Zahlen.

Wir bezeichnen mit $r$ den nichteuklidischen Kreis mit dem Mittelpunkt in $z=0$ und den (hyperbolischen) Radius $\delta$, und

$$
\bar{a}=\sup \{K(z) \mid z \in \gamma\}, \quad \omega=1_{\gamma}^{*} .
$$

Es sei $\lambda$ ein Kontinuum, das den Punkt $z=0$ enthält, und dessen hyperbolischen Durchmesser gleich $\delta$ ist, und

$$
1 \geq a_{\lambda}=\inf \left\{1_{\lambda}^{*}(z) \mid z \in \gamma\right\}>0 .
$$

Man kann zeigen, dass

$$
a=\inf \left\{a_{\lambda} \mid \lambda \text { wie oben }\right\}>0
$$

ist. Wir setzen

$$
\underline{a}=a \inf \{K(z) \mid z \in \gamma\} .
$$

Wir bezeichnen mit $T_{n}$ die eineindeutige und konforme Selbstabbildung des Kreises $R$, die den Punkt $z=1$ fest lässt und den Punkt $z_{n}$ in 0 überführt:

$$
T_{n}(z)=\frac{z-z_{n}}{1-z \bar{z}_{n}} \cdot \frac{1-\bar{z}_{n}}{1-z_{n}}
$$

$K \circ T_{n}^{-1}$ ist minimal und deshalb ist $K \circ T_{n}^{-1}=K\left(z_{n}\right) K$. Es ist

$$
K_{\gamma_{n}}^{*} \circ T_{n}^{-1}=\left(K^{\circ} \circ T_{n}^{-1}\right)_{\lambda_{n}}^{*}={ }^{\circ} K\left(z_{n}\right) K_{\lambda_{n}}^{*},
$$

wo $\lambda_{n}=T_{n}\left(\gamma_{n}\right)$ ist. Wir haben $a \omega \leq 1_{\lambda n}^{*}$ und somit

$$
\underline{a} \omega \leq \inf \{K(z) \mid z \in \gamma\} 1_{\lambda_{n}}^{*} \leq K_{\lambda_{n}}^{*} \leq \bar{a} \omega .
$$

Daraus folgt

$$
\underline{a} K\left(z_{n}\right) \omega \leq K_{\gamma_{n}}^{*} \circ T_{n}^{-1} \leq \bar{a} K\left(z_{n}\right) \omega .
$$

Schreiben wir diese Ungleichungen im Punkte $T_{n}(0)$, so erhalten wir

$$
\underline{a} K\left(z_{n}\right) \omega\left(T_{n}(0)\right) \leq K_{r_{n}}^{*}(0) \leq \bar{a} K\left(z_{n}\right) \omega\left(T_{n}(0)\right) .
$$

Es ist aber

$$
\begin{aligned}
& \omega\left(T_{n}(0)\right)=\omega\left(-z_{n} \frac{1-\bar{z}_{n}}{1-z_{n}}\right)=\omega\left(\left|-z_{n} \frac{1-\bar{z}_{n}}{1-z_{n}}\right|\right)=\omega\left(r_{n}\right), \\
& K\left(z_{n}\right)=\frac{1-r_{n}^{2}}{\left(1-r_{n}\right)^{2}+4 r_{n} \sin ^{2} \frac{\theta_{n}}{n}},
\end{aligned}
$$


wo wir $z_{n}=r_{n} e^{i \theta_{n}}$ gesetzt haben. Daraus folgt

$$
\underline{a} \frac{2}{1+\alpha^{2}}\left|\frac{\partial \omega}{\partial r}\right|_{r=1} \leq \lim _{n \rightarrow \infty} K_{\Upsilon_{n}}^{*}(0) \leq \varlimsup \lim K_{\Upsilon_{n}}^{*}(0) \leq \bar{a} \frac{2}{1+\alpha^{2}}\left|\frac{\partial \omega}{\partial r}\right|_{r=1}
$$

und somit auch die gesuchten Ungleichungen.

Es sei $z_{n} \rightarrow \succ e^{i \theta}$ und $\zeta_{n}$ ein Punkt, dessen hyperbolische Entfernung von $z_{n}$ kleiner als $\delta$ ist. Dann ist auch $\zeta_{n} \rightarrow \succ^{i \theta}$. In der Tat, sei

$$
\alpha^{\prime}=\varlimsup_{n \rightarrow \infty} \frac{\left|\theta-\arg \zeta_{n}\right|}{1-\left|\zeta_{n}\right|}
$$

und $\gamma_{n}$ ein nichteuklidischer Kreis mit dem Durchmesser $\delta$, der die Punkte $z_{n}$ und $\zeta_{n}$ enthält. Indem wir zu einer Teilfolge übergehen, können wir annehmen, dass

$$
\begin{aligned}
& \alpha^{\prime}=\lim _{n \rightarrow \infty} \frac{\left|\theta-\arg \zeta_{n}\right|}{1-\left|\zeta_{n}\right|}, \\
& \alpha=\lim _{n \rightarrow \infty} \frac{\left|\theta-\arg z_{n}\right|}{1-\left|z_{n}\right|}<\infty
\end{aligned}
$$

ist. Nach dem Hilfssatz 14 ist

$$
0<\frac{\underline{c}_{\delta}}{1+\alpha^{2}} \leq \lim _{n \rightarrow \infty} K_{\Upsilon_{n}}^{*}(0) \leq \varlimsup_{n \rightarrow \infty} K_{\Upsilon_{n}}^{*}(0) \leq \frac{\bar{c}_{\delta}}{1+\alpha^{\prime 2}}
$$

woraus $\alpha^{\prime}<\infty$ folgt.

Hilfssatz 15. Es sei $\left\{z_{n}\right\}$ eine Folge, für die $z_{n} \rightarrow \succ z_{0}=1$, und $\gamma_{n}$ wie im Hilfssatz 14. Dann ist $z_{0}=1 \notin \Delta_{1}\left(R-\bigcup_{n=1}^{\infty} \gamma_{n}\right)$.

Es ist $K_{\curlyvee}^{*} \geq K_{\Upsilon_{n}}^{*}, \gamma=\bigcup_{n=1}^{\infty} \gamma_{n}$. Wir wählen eine konvergente Teilfolge der Folge $\left\{K_{r_{n}}^{*}\right\}$. Nach Hilfssatz 14 ist die Grenzfunktion, die wir mit $u$ bezeichnen werden nicht Null. Aus $u \leq K_{\Upsilon}^{*}$ folgt

$$
\begin{aligned}
& K_{\Upsilon}^{*}=\left(K_{\Upsilon}^{*}\right)_{\curlyvee}^{*}=u_{\curlyvee}^{*}+\left(K_{\curlyvee}^{*}-u\right)_{\curlyvee}^{*} \leq u_{\Upsilon}^{*}+K_{\curlyvee}^{*}-u, \\
& u \leq u_{\curlyvee}^{*} \leq u .
\end{aligned}
$$

Aus $0<u \leq K$ folgt $u=\alpha K(0<\alpha \leq 1)$ und

$$
K_{\curlyvee}^{*}=\frac{1}{\alpha} u_{\curlyvee}^{*}=\frac{1}{\alpha} u=K
$$

Nach Hilfssatz 4 ist

$$
I_{G} K=K-K_{\curlyvee}^{*}=0
$$


wo wir $G=R-r$ gesetzt haben.

SATZ $21^{16)}$. Ist $R^{\prime}$ nicht der Riemannschen Kugel $(|w| \leq \infty)$ oder der endlichen Ebene $(|w|<\infty)$ konform äquivalent, so ist $\mathfrak{F}(f) \subset \mathfrak{F}^{*}(f)$ und $f^{*}=\hat{f}$ auf $\mathfrak{F}(f)$. Ist $R^{\prime}$ die endliche Ebene, so hat $f$ in jedem Punkt $e^{i \theta} \in \mathfrak{F}(f)$, für welchen $\hat{f}\left(e^{i \theta}\right) \neq \infty$ ist (d.h. nach Satz 16 fast überall auf $\left.\mathfrak{F}(f)\right)$ den Winkelgrenzpunkt $\hat{f}\left(e^{i \theta}\right)$.

Wir führen für jeden Punkt $\hat{p}^{\prime} \in \hat{R}^{\prime}$ eine Klasse von offenen Mengen aus $R^{\prime}, \mathbb{E}_{p^{\prime}}$, ein, für die

$$
\bigcap_{G^{\prime} \in \mathfrak{E}_{\hat{p}}} \bar{G}^{\prime}=\left\{\hat{p}^{\prime}\right\}
$$

ist. Ist $\hat{p}^{\prime}$ ein innerer Punkt von $R^{\prime}\left(p^{\prime} \in R^{\prime}\right)$, dann bestehe $\mathfrak{F}_{p^{\prime}}$ aus allen Kreisscheiben von $R^{\prime}$, die den Punkt $\hat{p}^{\prime}$ enthalten. Ist $\hat{p}^{\prime}$ ein Punkt von $\Delta^{\prime}$, und besteht $\Delta^{\prime}$ aus diesem einzigen Punkt, so soll $\mathscr{F}_{\hat{p}^{\prime}}=\left\{R^{\prime}-R_{n}^{\prime}\right\}$ sein, wobei $\left\{R_{n}^{\prime}\right\}$ eine normale Ausschöpfung von $R^{\prime}$ ist, so dass der Rand von $R_{n}^{\prime}$ aus einer einzigen Jordankurve besteht, und das Geschlecht von $R_{n}^{\prime}$ nicht Null ist. Ist $\hat{p}^{\prime}$ ein Punkt von $\Delta^{\prime}$ und enthält $\Delta^{\prime}$ auch andere Punkte, so soll $\mathbb{F}_{\hat{p}^{\prime}}$ aus den Mengen $G^{\prime}=\hat{G}^{\prime} \cap R^{\prime}$ bestehen, wo $\hat{G}^{\prime}$ eine derartige Umgebung von $\hat{p}^{\prime}$ ist, dass $R^{\prime}-G^{\prime}$ keine kompakte Komponente hat. Für die ersten zwei Fälle ist obige Bedingung offenbar erfüllt. Um das auch im letzen Falle zu beweisen, zeigen wir zuerst folgendes: es sei $\hat{D}^{\prime}$ eine beliebige Umgebung von $\hat{p}^{\prime}, F^{\prime}=R^{\prime}-\hat{D}^{\prime}$, $F_{0}^{\prime}$ eine kompakte Komponente von $F^{\prime}$ und $p_{0}^{\prime} \in F_{0}^{\prime}$. Es sei $\left\{p_{n}^{\prime}\right\}$ eine Folge auf $F^{\prime}\left(p_{n}^{\prime} \neq p_{0}^{\prime}\right)$, die gegen $p_{0}^{\prime}$ konvergiert und $F_{n}^{\prime}$ die Komponente von $F^{\prime}$, die den Punkt $p_{n}^{\prime}$ enthält. Dann können nicht alle $F_{n}^{\prime}$ nichtkompakt sein. In der Tat, es sei $\Omega^{\prime}$ ein relativkompaktes Gebiet, das $F_{0}^{\prime}$ enthält, $\underline{F}_{n}^{\prime}$ die Komponente von $F_{n}^{\prime} \cap \Omega^{\prime}$ die den Punkt $p_{n}^{\prime}$ enthält und $\underline{F}_{0}^{\prime}$ das obere topologische Limes der Folge $\left\{\underline{F}_{n}^{\prime}\right\}$, d.h. $\underline{F}_{0}^{\prime}$ ist die Menge der Punkte $q^{\prime} \in \Omega^{\prime}$, für die jede Umgebung von $q^{\prime}$ in $\Omega^{\prime}$ einen nichtleeren Durchschnitt mit unendlich vielen Mengen $\underline{F}_{n}^{\prime}$ hat. Es ist $\underline{F}_{0}^{\prime} \subset F^{\prime}, p_{0}^{\prime} \in \underline{F}_{0}^{\prime}$ und $\underline{F}_{0}^{\prime}$ zusammenhängend. Daraus folgt $\underline{F}_{0}^{\prime} \subset F_{0}^{\prime}$. Da die Mengen $F_{n}^{\prime}$ nichtkompakt sind, so gibt es einen Punkt $q_{n}^{\prime} \in \underline{F}_{n}^{\prime} \cap \partial \Omega^{\prime}$. Es sei $q^{\prime}$ ein Häufungspunkt der Folge $\left\{q_{n}^{\prime}\right\}$. Er gehört gleichzeitig der Menge $\underline{F}_{0}^{\prime}$ und $\partial \Omega^{\prime}$ an. Daraus folgt, dass $F_{0}^{\prime}$ mit $\partial \Omega^{\prime}$ einen gemeinsamen Punkt

16) Dieser Satz ist auch dann gültig, wenn $f$ blos eine innere Abbildung ist ([15] seite 107 ). 
hat, entgegen der Voraussetzung $F_{0}^{\prime} \subset \Omega^{\prime}$. Die Mengen $F_{n}^{\prime}$ können also nicht alle nichtkompakt sein. Indem man zu einer Teilfolge der Folge $\left\{F_{n}^{\prime}\right\}$ übergeht, folgert man, dass nur endlich viele von ihnen nichtkompakt sein können. Fügen wir also der Menge $\hat{D}^{\prime}$ alle kompakten Komponenten der Menge $F^{\prime} z u$, so entsteht eine offene Menge $\hat{G}^{\prime}$, für die $\hat{G}^{\prime} \cap R^{\prime} \in \mathbb{E} \hat{p}^{\prime}$. Es ist aber

$$
\overline{\hat{D}} \cap \Delta^{\prime}=\overline{\hat{G}^{\prime} \cap R^{\prime} \cap \Delta^{\prime}}
$$

und somit

$$
\left(\bigcap_{G^{\prime} \in \mathfrak{E}_{\hat{p}} \hat{G}^{\prime}} \overline{\bar{G}^{\prime}}\right) \cap \Delta^{\prime}=\left\{\hat{p}^{\prime}\right\} .
$$

Es sei $q^{\prime} \in R^{\prime}$ und $s^{\prime} \in \Delta_{1}^{\prime}, s^{\prime} \neq \hat{p}^{\prime}$. Da $s^{\prime}$ ein erreichbarer Randpunkt von $R^{\prime}$ ist, so gibt es eine Kurve $\lambda$ die $q^{\prime}$ mit $s^{\prime}$ in $R^{\prime}$ vereinigt. Es sei $F$ eine abgeschlossene Umgebung von $q^{\prime}$ und

$$
G^{\prime}=R^{\prime}-\lambda-F
$$

$G^{\prime}$ gehört der Klasse $\mathfrak{E}_{\hat{p}^{\prime}}$ an und $\bar{G}^{\prime}$ enthält den Punkt $q^{\prime}$ nicht. Da $q^{\prime}$ beliebig auf $R^{\prime}$ war, so ist

$$
\bigcap_{G^{\prime} \in \mathfrak{E} \hat{p}^{\prime}} \overline{G^{\prime}}=\left(\bigcap_{G^{\prime} \in \mathfrak{E} \hat{p}^{\prime}} \overline{G^{\prime}}\right) \cup \Delta^{\prime}=\left\{\hat{p}^{\prime}\right\} .
$$

Es sei $e^{i \theta} \in \tilde{F}(f), \hat{G}^{\prime}$ eine Umgebug von $\hat{f}\left(e^{i \theta}\right)$, derart dass $G^{\prime}=\hat{G}^{\prime} \cap R^{\prime}$ $\in E_{\hat{p}\left(e^{i} \theta\right)}$ und $G=f^{-1}\left(G^{\prime}\right)$. Wir werden beweisen, dass $R-G$ keine kompakte Komponente enthält. Es ist $R-G=f^{-1}\left(R^{\prime}-G^{\prime}\right)$. Enthält $R^{\prime}-G^{\prime}$ keine kompakte Komponente, so ist das offenbar richtig. Enthält aber $R^{\prime}-G^{\prime}$ eine kompakte Komponente, so ist $\hat{p}^{\prime}=\hat{f}\left(e^{i \theta}\right)$ ein Punkt einer abgeschlossenen Fläche oder der einzige Punkt des idealen Randes von $R^{\prime}$. In beiden Fällen ist $R^{\prime}-G^{\prime}$ ein abgeschlossenes Gebiet (triangulierbar) mit einer positiven EulerPoincaréschen Charakteristik $\rho^{\prime}$, denn der Fall der Riemannschen Kugel und der Fall der endlichen Ebene und $\hat{f}\left(e^{i \theta}\right)=\infty$ wurden ausgeschlossen. Wir nähmen an, dass $R-G$ eine kompakte Komponente $\Omega$ enthält. Es sei $\rho$ die Charakteristik von $\Omega$ und $n$ der Grad der Überlagerung von $R^{\prime}-G^{\prime}$ durch $\Omega$. Aus der Hurwitzschen Relation folgt $\rho \geq n \rho^{\prime} \geq n$. Es sei $l$ die Zahl der Randkomponenten von $\Omega$. Es ist $\rho=l-2$ und, da $R^{\prime}-G^{\prime}$ eine einzige Randkomponente hat, auch $l \leq n$, woraus die widersprechende Beziehung

$$
n-2 \geq n
$$


folgt. $R-G$ enthält also keine kompakte Komponente.

Es sei $\left\{z_{n}\right\}$ eine Folge aus $R-G$ die gegen $e^{i \theta}$ strebt, $\delta$ eine beliebige positive Zahl und $\gamma_{n}$ ein Kontinuum, das den Punkt $z_{n}$ enthält, den hyperbolischen Durchmesser gleich $\delta$ hat und in $R-G$ enthalten ist. Die Existenz von $\gamma_{n}$ ergibt sich aus der Tatsache, dass alle Komponenten von $R-G$ nichtkompakt sind. Hat die Folge $\left\{z_{n}\right\}$ im Punkte $e^{i \theta}$ einen Winkeleingang, so folgt aus dem Hilfssatz $15 e^{i \theta} \notin \Delta_{1}\left(R-\bigcup_{n=1}^{\infty} \gamma_{n}\right)$. Desto mehr ist $e^{i \theta} \notin \Delta_{1}(G)$, da $G \subset R-\bigcup_{n=1}^{\infty} \gamma_{n}$ ist, was der Definition von $\hat{f}$ und der Eigenschaft a) von $\hat{M}$ widerspricht. Für jede Folge $\left\{z_{n}\right\}$, die in $e^{i \theta}$ einen Winkeleingang hat, muss also $f\left(z_{n}\right)$ für genügend grosse $n$ in $G^{\prime}$ enthalten sein. Da aber

$$
\bigcap_{G^{\prime} \in \mathfrak{E} \hat{f}\left(e^{i \theta}\right)} \overline{G^{\prime}}=\left\{\hat{f}\left(e^{i \theta}\right)\right\}
$$

ist, so folgt, dass $\dot{f}$ im Punkte $e^{i \theta}$ den Winkelgrenzpunkt $\hat{f}\left(e^{i \theta}\right)$ hat, was zu beweisen war.

Im Falle $R^{\prime}=\{|w| \leq \infty\}$, der aus diesem Satz ausgeschlossen wurde, ist die Inklusion $\mathfrak{F}(f) \subset \mathfrak{F}^{*}(f)$ nicht mehr wahr. Wir werden später (siehe Seite 74 und den Anhang) ein Beispiel in dieser Richtung geben.

HilfsSATZ 16. Es sei $R^{\prime}$ die Riemannsche Kugel, $w_{0} \in R^{\prime}$ und $e^{i \theta}$ ein Punkt in welchem die Menge $f^{-1}\left(w_{0}\right)$ keinen Winkeleingang hat. Ist $e^{i \theta} \in \mathfrak{F}(f)$ und $\hat{f}\left(e^{i \theta}\right) \neq w_{0}$, so hat $f$ in $e^{i \theta}$ den Winkelgrenzpunkt $\hat{f}\left(e^{i \theta}\right)$.

Es sei $G^{\prime}$ eine Kreisscheibe, die den Punkt $\hat{f}\left(e^{i \theta}\right)$ enthält aber nicht den Punkt $w_{0}$, und es sei $G=f^{-1}\left(G^{\prime}\right)$. Die Menge $R-G$ hat in $e^{i \theta}$ keinen Winkeleingang. In entgegengesetzten Falle sei $\left\{z_{n}\right\}$ eine Folge aus $R-G$, für die $z_{n} \rightarrow \succ e^{i \theta}$. Wir bezeichnen mit $\gamma_{n}$ die Komponente der Menge $R-G$ die den Punkt $z_{n}$ enthält, mit $\delta$ eine positive Zahl und mit $\mathfrak{N}$ die Menge der natürlichen Zahlen, für die der nichteuklidische Durchmesser von $\gamma_{n}$ grösser als $\delta$ ist. Wäre $\Re$ unendlich, so wäre laut des Hilfssatzes $15, e^{i \theta} \notin \Delta_{1}\left(R-\bigcup_{n \in \Re} \gamma_{n}\right)$. Es ist aber $G \subset R-\bigcup_{n \in \mathfrak{N}} \gamma_{n}$ und somit $e^{i \theta} \notin \Delta_{1}(G)$, was widersprechend ist. $\mathfrak{N}$ ist also endlich. Für $n \notin \Re$ ist $\gamma_{n}$ kompakt und deshalb enthält $\gamma_{n}$ einen Punkt $\zeta_{n}$, $f\left(\zeta_{n}\right)=w_{0}$. Da die hyperbolische Entfernung zwischen $\zeta_{n}$ und $z_{n}$ nicht grösser als $\delta$ ist, so hat die Folge $\left\{\zeta_{n}\right\}$ einen Winkeleingang in $e^{i \theta}$ entgegen der Voraussetzung des Hilfssatzes. Also hat $R-G$ in $e^{i \theta}$ keinen Winkeleingang. 
Da $G^{\prime}$ beliebig war folgt sofort, dass $f$ im Punkte $e^{i \theta}$ den Winkelgrenzpunkt $\hat{f}\left(e^{i \theta}\right)$ hat.

SAtz 22. Es sei $R^{\prime}$ die Riemannsche Kugel. Existiert ein $w_{0} \in R^{\prime}$ derart, dass

$$
\sum_{f\left(z_{n}\right)=u_{0}}\left(1-\left|z_{n}\right|\right)<\infty
$$

ist, so ist $f^{*}$ fast überall auf $\tilde{F}(f)$ definiert und gleich $\hat{f}$.

Es sei $A_{1}$ die Menge der Punkte $e^{i n} \in \mathfrak{F}(f)$, wo $\left\{z_{n}\right\}$, einen Winkeleingang hat. Wir setzen

$$
G\left(e^{i \theta}, \alpha\right)=\left\{z\left|\frac{1}{2}<\right| z|<1,| \arg \frac{e^{i \theta}-z}{e^{i \theta}} \mid<\alpha\right\} \quad\left(\alpha<\frac{\pi}{2}\right) .
$$

Enthält $G\left(e^{i \theta}, \alpha\right)$ den Punkt $z_{n}=r_{n} e^{i \theta_{n}}$, so ist

$$
\left|\theta_{n}-\theta\right|<\left(1-r_{n}\right) \operatorname{tg} \alpha \text {. }
$$

Es sei $B_{\alpha}$ die Menge der Punkte $e^{i \theta}$, für die $G\left(e^{i \theta}, \alpha\right)$ unendlich viele $z_{n}$ enthält ; dann ist

$$
\chi\left(B_{\alpha}\right) \leq \operatorname{tg} \alpha \sum_{n=m}^{\infty}\left(1-r_{n}\right)<\infty
$$

für ein beliebiges $m$, und somit ist $B_{\alpha}$ vom Lebesgueschen Masse Null. Da aber $A_{1} \subset \bigcap_{\alpha<\pi / 2} B_{\alpha}$ ist, so ist $A_{1}$ vom Lebesgueschen Masse Null.

Es sei

$$
A_{2}=\left\{e^{i \theta} \in \mathfrak{F}(f) \mid \hat{f}\left(e^{i \theta}\right)=w_{0}\right\} .
$$

Aus dem Satz 16 ist $\mathrm{zu}$ ersehen, dass auch $A_{2}$ vom Lebesgueschen Masse Null ist. Ist $e^{i \theta} \in \mathfrak{F}(f)-A_{1}-A_{2}$, so folgt aus dem Hilfssatz 16 , dass $f$ in $e^{i 9}$ den Winkelgrenzwert $\hat{f}\left(e^{i \theta}\right)$ hat.

Folgesatz 5. Es sei $R^{\prime}$ die Riemannsche Kugel und $f$ eine Lindelöfsche Abbildung (beschänktartig). Dann ist $f^{*}$ fast überall auf $|z|=1$ definiert und gleich $\hat{f}$.

Nach Satz 18 ist $\hat{f}$ fast überall definiert und, da die Bedingung

$$
\sum_{f\left(x_{n}\right)=w_{0}}(1-|z|)<\infty
$$

für alle Punkte $w_{0} \in R^{\prime}$ erfüllt ist, erhalten wir sofort dẹn Folgesatz aus dẹm Satz 22, 
Dieser Folgesatz ist gerade der Satz von Fatou-Nevanlinna. Die Eigenschaft e) von $\hat{f}$ erlaubt uns die Behauptung, dass $f$ fast überall einen Winkelgrenzpunkt hat, etwas $z u$ verschärfen. Man kann nähmlich sagen, dass fast alle $e^{i \theta}$ folgende Eigenschaft besitzen: Es sei $F$ eine abgeschlossene Menge in $R$, für die $e^{i \theta} \notin \Delta_{1}(R-F)$ ist ( $F$ kann diese Bedingung erfüllen, ohne im Punkte $e^{i \theta}$ einen Winkeleingang zu haben); man kann dann eine Folge $\left\{z_{n}\right\}$ auf $F$ finden, die gegen $e^{i \theta}$ konvergiert, und für die $\left\{f\left(z_{n}\right)\right\}$ gegen $\hat{f}\left(e^{i \theta}\right)$ konvergiert.

\section{Fatousche Abbildungen}

Der Abbildung $\hat{f}$ fehlt die Kompositionseigenschaft, d.h. es ist nicht immer $\widehat{f^{\prime} \circ f}=\hat{f}^{\prime} \circ \hat{f} . \quad$ Da diese Eigenschaft bei einigen Anwendungen wichtig ist, werden wir eine Teilmenge der Menge $\mathfrak{F}(f)$ einführen, auf der die Abbildung $\hat{f}$ diese Eigenschaft besitzt. Wir bezichnen, in Falle $R^{\prime} \notin \mathrm{O}_{G}$,

$$
\widetilde{\mho}_{0}(f)=\left\{s \in \Delta_{1}(f) \mid E K_{s}<\infty\right\} .
$$

Nach der Eigenschaft $(f)$ der Abbildung $\hat{f}$ ist $\widetilde{\mho}_{0}(f) \subset \mathfrak{F}(f)$. Die Menge $\widehat{\mho}_{0}(f)$ besitzt folgende Eigenschaften.

a) $\tilde{F}_{0}(f)$ ist eine Borelsche Menge.

b) Es sei $s^{\prime} \in \Delta^{\prime} ; \widetilde{\vartheta}_{0}(f) \cap \hat{f}^{-1}\left(s^{\prime}\right)$ ist dann und nur dann leer, wenn IK $K^{\prime} s^{\prime}$ total nichtdiskret ist.

c) Ist $s \in \widetilde{F}_{0}(f)$ und $\hat{f}(s) \in \Delta_{1}^{\prime}\left(G^{\prime}\right)$ für eine offene Menge $G^{\prime} \subset R^{\prime}$, so ist auch $s \in \Delta_{1}\left(f^{-1}\left(G^{\prime}\right)\right)$.

a) Nach Satz 4 ist die Funktion $s \rightarrow E K_{s}\left(p_{0}^{\prime}\right)$ halbstetig. Daraus und aus der Tatsache, dass $\Delta_{1}(f)$ eine Borelsche Menge ist folgt sofort, dass auch $\mathfrak{F}_{0}(f)$ eine Borelsche Menge ist.

b) Ist $I K^{\prime} s^{\prime}$ nicht total nichtdiskret, so gibt es einen Punkt $s \in \Delta_{1}$, und eine positive Zahl $\alpha$, derart dass

$$
\alpha K_{s} \leq I K_{S^{\prime}}^{\prime} \leq K_{s^{\prime}}^{\prime} \circ f
$$

ist. Dann ist offenbar $s$ in $\mathfrak{F}_{0}(f)$ enthalten und $s \in \hat{f}^{-1}\left(s^{\prime}\right)$. Ist, umgekehrt, $s \in \hat{f}^{-1}\left(s^{\prime}\right) \cap \hat{\mho}_{0}(f)$, so ist nach der Eigenschaft $\left.f\right)$ von $\hat{f}$

$$
E K_{s}=\alpha K_{s^{\prime}}^{\prime}
$$

wo $\alpha$ eine positive Zahl ișt. Dạaụ und aus der Eigenschaft c) von $\hat{f}$ folgt 


$$
{ }_{\alpha}^{1} K_{s} \leq I K_{s^{\prime}}^{\prime}
$$

und $I K_{S^{\prime}}^{\prime}$ ist nicht total nichtdiskret.

c) Es ist nach der Eigenschaft f) von $\hat{f}$

$$
E_{f} K_{s}=\alpha K^{\prime} \hat{f}^{(s)} .
$$

$\mathrm{Da}$

$$
I_{f} K^{\prime} \hat{f}(s) \geq \frac{1}{\alpha} K_{s} \neq 0, \quad I_{G^{\prime}} K^{\prime} \hat{f}^{(s)} \neq 0
$$

ist, so ist nach Hilfssatz 6

$$
\underset{f^{-1}\left(G^{\prime}\right)}{E} \underset{f^{-1}\left(G^{\prime}\right)}{I} I_{f} K^{\prime} \hat{f}^{(s)}=I_{f} K^{\prime} \hat{f}(s) \geq \frac{1}{\alpha} K_{s} .
$$

Nach Hilfssatz 3 ist dann

$$
\underset{f^{-1}\left(G^{\prime}\right)}{E} \underset{f^{-1}\left(G^{\prime}\right)}{I} K_{s}=K_{s}
$$

und $s \in \Delta_{1}\left(f^{-1}\left(G^{\prime}\right)\right)$.

HilfSSATZ 17. Es sei $R \stackrel{f}{\longrightarrow} R^{\prime} \stackrel{f^{\prime}}{\longrightarrow} R^{\prime \prime}, R^{\prime} \notin O_{G}$ und $s \in \widetilde{\jmath}_{0}(f)$. s gehört der Menge $\widetilde{F}\left(f^{\prime} \circ f\right)$ dann und nur dann an, wenn $\hat{f}(s)$ der Menge $\widetilde{F}\left(f^{\prime}\right)$ angehört. In "iesem Falle ist

$$
\hat{f}^{\prime}(\hat{f}(s))=\widehat{f^{\prime} \circ f}(s) .
$$

Es sei $\hat{f}(s) \in \mathfrak{F}\left(f^{\prime}\right)$ und $\hat{G}^{\prime \prime}$ eine Umgebung von $\hat{f}^{\prime}(\hat{f}(s))$. Wir bezeichnen

$$
\begin{aligned}
& G^{\prime}=f^{\prime-1}\left(\hat{G}^{\prime \prime} \cap R^{\prime \prime}\right), \\
& G=\left(f^{\prime} \circ f\right)^{-1}\left(\hat{G}^{\prime \prime} \cap R^{\prime \prime}\right)=f^{-1}\left(G^{\prime}\right) .
\end{aligned}
$$

Nach der Definition von $\hat{f}^{\prime}$ ist $\hat{f}(s) \in \Delta_{1}\left(G^{\prime}\right)$ und nach der Eigenschaft c) von $\widetilde{\mho}_{0}(f)$ ist $s \in \Delta_{1}(G) . \quad \hat{M}_{f^{\prime} \circ f}(s)$ reduziert sich somit auf den Punkt $\hat{f}^{\prime}(\hat{f}(s))$ und $s \in \mathfrak{F}\left(f^{\prime} \circ f\right)$,

$$
\widehat{f^{\prime} \circ f(s)}=\hat{f}^{\prime}(\hat{f}(s))
$$

Es sei jetzt $s \in \widetilde{F}\left(f^{\prime} \circ f\right)$ und $\hat{G}^{\prime \prime}$ eine Umgebung von $\widehat{f^{\prime} \circ f}(s)$. Wir setzen $G^{\prime}$ und $G$ wie oben. Nach der Eigenschaft $f$ ) von $f^{\prime}$ ist

$$
E_{f} K_{s}=\alpha K^{\prime} \hat{f}(s), \quad I_{f} K^{\prime} \hat{f}^{(\varsigma)} \geq \frac{1}{\alpha} K_{s}
$$


wo $\alpha$ eine positive $\mathrm{Zahl}$ ist. $\mathrm{Da} s \in \Delta_{1}(G)$ ist, so ist

$$
I_{G} I_{f} K^{\prime} \hat{f}^{\prime s)} \geq \frac{1}{\alpha} I K_{G} \neq 0
$$

Nach Satz 12 ist

$$
I_{f_{0}} I K_{G^{\prime}}^{\prime} K_{\hat{f}(s)}=I_{\sigma} I_{f} K^{\prime} \hat{f}(s) \neq 0,
$$

wo $f_{0}$ die Abbildung von $G$ in $G^{\prime}$, die mit $f$ zusammenfällt ist. Daraus folgt

$$
I_{G^{\prime}} K^{\prime} \hat{f}(s) \neq 0
$$

und $\hat{f}(s) \in \Delta_{1}\left(G^{\prime}\right)$. Die Menge $\hat{M}_{f^{\prime}}(\hat{f}(s))$ reduziert sich somit auf den Punkt

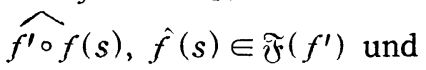

$$
\hat{f}^{\prime}(\hat{f}(s))=\widehat{f^{\prime} \circ}(s) .
$$

Es sei $\nu_{f}\left(p^{\prime}\right)$ die Zahl der Punkte der Menge $f^{-1}\left(p^{\prime}\right)$ und

$$
R_{k}^{\prime}=\left\{p^{\prime} \in R^{\prime} \mid \nu f\left(p^{\prime}\right) \geq k\right\} \quad(k<\infty) .
$$

$R_{k}^{\prime}$ ist eine offene Menge und $R_{k+1}^{\prime} \subset R_{k}^{\prime}$.

HiLfSSATZ 18. Ist $s^{\prime} \in \Delta_{1}^{\prime}(f)-\bigcap_{k=1}^{\infty} \Delta_{1}^{\prime}\left(R_{k}^{\prime}\right)$, so ist $\hat{f}^{-1}\left(s^{\prime}\right) \cap \mathfrak{\mho}_{0}(f)$ nicht leer, I $K_{s^{\prime}}^{\prime}$ ist diskret und

$$
I K_{s^{\prime}}^{\prime}=\int_{\hat{f}^{-1}\left(s^{\prime}\right) \cap \mathcal{F}_{0}(f)} K_{s} d\left(\delta_{s^{\prime}} \circ f\right)(s)=\sum_{\substack{\hat{f}\left(s_{i}\right)=s^{\prime} \\ s_{l} \in \mathfrak{F}_{0}(f)}} \alpha_{i} K_{s_{i}},
$$

wobei die letzte Summe endlich viele Glieder hat. Ist $s^{\prime} \in \Delta_{1}^{\prime}\left(R_{k+1}^{\prime}\right)$, so besteht $\hat{f}^{-1}\left(s^{\prime}\right) \cap \mho_{0}(f)$ aus höchstens $k$ Punkten.

Nach Satz 14 ist

$$
I_{f} K_{s^{\prime}}^{\prime}=\int_{\Delta_{1}(f)} K_{s} d\left(\delta_{s^{\prime}} \circ f\right)(s)
$$

Angenommen $I_{f} K_{s^{\prime}}^{\prime}$ wäre nicht diskret. Dann kann man in $\Delta_{1}(f) k$ abgeschlossene paarweise punktfremde Mengen $A_{1}, A_{2}, \ldots, A_{k}$ finden, so dass $\left(\delta_{s} \circ f\right)\left(A_{i}\right)>0(i=1,2, \ldots, k)$ ist. Es seien $\hat{G}_{i}(i=1,2, \ldots, k)$ paarweise puntfremde Umgebungen der Mengen $A_{i}$ und $G_{i}=\hat{G}_{i} \cap R . \quad$ Da $A_{i} \subset A_{1}\left(G_{i}\right)$ ist, so ist nạch dẹm Sạț $15^{\prime}$ 


$$
\underset{G_{i} G_{i}}{\operatorname{EI}} I_{f} K_{s^{\prime}}^{\prime} \geq \int_{A_{i}} K_{s} d\left(\delta_{s^{\prime}} \circ f\right)(s)>0
$$

und somit ist

$$
\underset{a_{i}}{I} I_{f} K_{s^{\prime}}^{\prime} \neq 0 .
$$

Wir bezeichnen $G_{i}^{\prime}=f\left(G_{i}\right)$ und es sei $f_{i}$ die Abbildung von $G_{i}$ in $G_{i}^{\prime}$, die mit $f$ zusammenfällt. Dann ist nach Satz 12

$$
I_{f_{i}} I K_{\alpha^{\prime} i} K_{S^{\prime}}^{\prime}=\underset{G_{i}}{I} I_{f} K_{S^{\prime}}^{\prime} \neq 0, \quad \underset{G^{\prime} i}{I} K_{s^{\prime}}^{\prime} \neq 0 .
$$

Laut des Hilfssatzes 10 ist $s \in \bigcap_{i=1}^{k} \Delta_{1}^{\prime}\left(G_{i}^{\prime}\right)=\Delta_{1}^{\prime}\left(\bigcap_{i=1}^{k} G_{i}^{\prime}\right)$. Es sei $p^{\prime} \in \bigcap_{i=1}^{k} G_{i}^{\prime}$. Dann kann man in jeder Menge $G_{i}$ einen Punkt $p_{i}$ finden, für welchen $f\left(p_{i}\right)=p^{\prime}$ ist. Es ist also $\nu_{f}\left(p^{\prime}\right) \geq k, \bigcap_{i=1}^{k} G_{i}^{\prime} \subset R_{k}^{\prime}$ und $s^{\prime} \in \Delta_{1}^{\prime}\left(R_{k}^{\prime}\right)$. Da aber $k$ beliebige war, so folgt $s^{\prime} \in \bigcap_{k=1}^{\infty} A_{1}\left(R_{k}^{\prime}\right)$, entgegen der Voraussetzung des Hilfssatzes. $I K_{s^{\prime}}^{\prime}$ ist also diskret :

$$
I K_{s^{\prime}}^{\prime}=\sum_{i} \alpha_{i} K_{s_{i}} \quad\left(\alpha_{i}>0\right) .
$$

Es ist offenbar $s_{i} \in \widetilde{F}_{0}(f)$ und $\hat{f}\left(s_{i}\right)=s^{\prime}$. Daraus folgt durch einfache Betrachtungen

$$
I K_{s^{\prime}}^{\prime}=\int_{\hat{f}^{-1}\left(s^{\prime}\right) \cap \widetilde{\mho}_{0}(f)} K_{s} d\left(\delta_{s^{\prime}}, \circ f\right)(s)=\sum_{\substack{\hat{f}\left(s_{i}\right)=s^{\prime} \\ s_{i} \ni \mathcal{F}_{0}(f)}} \alpha_{i} K_{s_{i}} .
$$

Ist $s^{\prime} \notin \Delta_{1}^{\prime}\left(R_{k+1}^{\prime}\right)$, so ergibt sich genau wie oben, dass $\hat{f}^{-1}\left(s^{\prime}\right) \cap \mathscr{F}_{0}(f)$ höchstens $k$ Punkte enthält.

SATZ 23. Es sei $A^{\prime} \subset \Delta_{1}^{\prime}-\bigcap_{k=1}^{\infty} \Delta_{1}^{\prime}\left(R_{k}^{\prime}\right)$ und

$$
u^{\prime}=\int_{A^{\prime}} K_{s^{\prime}}^{\prime} d \mu^{\prime}\left(s^{\prime}\right) \in H P\left(R^{\prime}\right)
$$

Dann ist

$$
I u^{\prime}=\int_{\hat{f}^{-1}\left(A^{\prime}\right) \cap \mathfrak{F}_{0}(f)} K_{s}^{\prime} d\left(\mu^{\prime} \circ f\right)(s) .
$$

Insbesondere ist

$$
I \omega\left(A^{\prime}, R^{\prime}\right)=\omega\left(\hat{f}^{-1}\left(A^{\prime}\right) \cap \mathfrak{F}_{0}(f), R\right) .
$$

Nach Hilfssatz 18 und Satz 14 ist

$$
I K_{S^{\prime}}^{\prime}=\int_{\hat{f}^{-1}\left(A^{\prime}\right) \cap \mathfrak{F}_{0}(f)} K_{s} d\left(\delta_{s^{\prime}} \circ f\right)(s),
$$


Daraus und aus dem Satz 14 folgt

$$
I u^{\prime}=\int_{\hat{f}^{-1}\left(A^{\prime}\right) \cap \mathfrak{F}_{0}(f)} K_{s} d\left(\mu^{\prime} \circ f\right)(s) .
$$

Folgesatz 6. Ist die von $f$ bestimmte Überlagerung endlich blättrig, d.h.

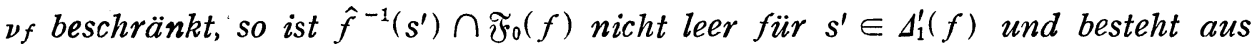
höchstens $k$ Punkten, wo $k=\sup \nu_{f}$ ist.

Es ist

$$
\begin{aligned}
& I \int_{\Delta^{\prime}} K_{s^{\prime}}^{\prime} d \mu^{\prime}\left(s^{\prime}\right)=\int_{\mathscr{\mho}_{0}(f)} K_{\supset} d\left(\mu^{\prime} \circ f\right)(s), \\
& I \omega\left(A^{\prime}, R^{\prime}\right)=\omega\left(\hat{f}^{-1}\left(A^{\prime}\right) \cap \mathfrak{F}_{0}(f), R\right) .
\end{aligned}
$$

Folgesatz 7. Ist $R^{\prime} \in U, f$ endlich-blättrig und $E I 1=1$, so ist $R \in U$. Ist $G^{\prime}$ eine offene Menge auf $R^{\prime}$, für die

$$
\underset{G^{\prime}}{E} I=1
$$

ist, so gehöhrt mindestens eine ihrer Komponente der Klasse U an.

Der letzte Teil dises Folgesatzes ist genau der Satz 12 von [1]; der hier angegebene Beweis benutzt aber nicht die universelle Überlagerungsfläche.

Da $R^{\prime} \in U$, so gibt es einen Punkt $s^{\prime} \in \Delta_{1}^{\prime}$ für welchen $K_{s^{\prime}}^{\prime}$ beschränkt ist. Aus $K_{s^{\prime}}^{\prime} \leq \alpha 1$ für eine positive Zahl $\alpha$ und aus Hilfssatz 3 folgt $E I K_{s^{\prime}}^{\prime}=K_{s^{\prime}}^{\prime}$ und somit ist

$$
I K_{S^{\prime}}^{\prime} \neq 0
$$

Nach Hilfssatz 18 ist

$$
I K_{s^{\prime}}^{\prime}=\sum_{\substack{\hat{f}\left(s_{i}\right)=s^{\prime} \\ s_{i} \in \widetilde{\mho}_{0}(f)}} \alpha_{i} K_{s_{i}}
$$

und alle $K_{s_{i}}$ müssen beschränkte Minimale sein, was den ersten Teil des Folgesatzes beweist. Der zweite folgt aus dem ersten durch evidente Betrachtungen.

HilfSSATZ 19. Ist $R \stackrel{f}{\longrightarrow} R^{\prime} \stackrel{f^{\prime}}{\longrightarrow} R^{\prime \prime}, f$ endlichblätrig und $\widehat{f^{\prime} \circ f}$ fast überall auf $\Delta_{1}(f)$ definiert, so ist auch $\hat{f}^{\prime}$ fast überall auf $\Delta_{1}^{\prime}(f)$ definiert.

Es ist $\mathrm{zu}$ beweisen, dass

$$
\chi_{R^{\prime}}\left(\Delta_{1}^{\prime}(f)-\mathfrak{F}\left(f^{\prime}\right)\right)=0
$$


ist. Nach Folgesatz 6 ist

$$
I_{f} \omega\left(\Delta_{1}^{\prime}(f)-\mathfrak{F}\left(f^{\prime}\right), R^{\prime}\right)=\omega\left(\hat{f}^{-1}\left(\Delta_{1}^{\prime}(f)-\mathfrak{F}^{\prime}\left(f^{\prime}\right)\right) \cap \mathfrak{F}_{0}(f), R\right) .
$$

Es sei $s \in \hat{f}^{-1}\left(\Delta_{1}^{\prime}(f)-\tilde{f}\left(f^{\prime}\right)\right) \cap \mathfrak{F}_{0}(f)$. Aus $\hat{f}(s) \notin \widetilde{F}\left(f^{\prime}\right)$ und Hilfssatz 17 folgt $s \notin \tilde{\jmath}\left(f^{\prime} \circ f\right)$. Es ist also

$$
\begin{aligned}
& \hat{f}^{-1}\left(\Delta_{1}^{\prime}(f)-\widetilde{F}\left(f^{\prime}\right)\right) \cap \widetilde{F}_{0}(f) \subset \Delta_{1}(f)-\widetilde{F}\left(f^{\prime} \circ f\right), \\
& \omega\left(\hat{f}^{-1}\left(\Delta_{1}^{\prime}(f)-\mathfrak{F}(f)\right) \cap \widetilde{F}_{0}(f), R\right) \leq \omega\left(\Delta_{1}(f)-\widetilde{F}\left(f^{\prime} \circ f\right), R\right)=0 .
\end{aligned}
$$

Mittels des Satzes 15 erhält man

$$
\omega\left(\Delta_{1}^{\prime}(f)-\widetilde{F}\left(f^{\prime}\right), R^{\prime}\right)=E_{f} I_{f} \omega\left(\Delta_{1}^{\prime}(f)-\mathfrak{F}\left(f^{\prime}\right), R^{\prime}\right)=0 .
$$

Hilfssatz 19'. Ist $G$ ein Gebiet auf $R, \eta$ die identische Abbildung von $G$ in $R$ und $f \circ \eta$ eine Lindelöfsche Abbildung, so ist $\hat{f}$ fast überall auf $\Delta_{1}(G)$ definiert.

Da $\eta$ einblättrig ist, so folgt dieser Hilfssatz unmittelbar aus dem Satz 18 und Hilfssatz 19.

Definition. Wir nennen $f$ eine Fatousche Abbildung, wenn man auf $R^{\prime}$ eine offene Menge $G^{\prime}$ finden kann, derart dass mindestens eine Komponente von $G^{\prime}$ eine Greensche Funktion besitzt und

$$
\underset{f^{-1}\left(G^{\prime}\right)}{E} \underset{f^{-1}\left(G^{\prime}\right)}{I} 1=1
$$

ist.

Es sei $F^{\prime}$ eine abgeschlossene Menge auf $R^{\prime}$ und $u^{\prime}$ eine harmonische Funtion auf $R^{\prime}-F^{\prime}$. Wir setzen

$$
G_{\alpha}^{\prime}=\left\{p^{\prime} \in R^{\prime}-F^{\prime} \mid u^{\prime}\left(p^{\prime}\right)>\alpha\right\}, \quad G_{\alpha}=f^{-1}\left(G_{\alpha}^{\prime}\right) .
$$

Ist, für ein $\alpha, \Delta_{1}-\Delta_{1}\left(G_{\alpha}\right)-\Delta_{1}\left(R-\bar{G}_{\alpha}\right)$ vom harmonischen Masse Null, so ist $f$ eine Fatousche Abbildung. Man kann nähmlich als Menge $G^{\prime}$ die Menge $G_{\alpha}^{\prime} \cup\left(R^{\prime}-\bar{G}_{\alpha}^{\prime}\right)$ setzen. Daraus folgt, dass jede Lindelöfsche Abbildung eine Fatousche Abbildung ist.

SATZ 24. $\hat{f}$ ist dann und nur dann fast überall auf $\Delta_{1}$ definiert, wenn $f$ eine Fatousche Abbildung ist.

Wir nehmen erst an, dass $f$ eine Fatousche Abbildung ist und es sei $G^{\prime}$ die in obiger Definition vorkommende Menge. Wir bezeichnnen mit $G_{i}$ die 
Komponenten von $f^{-1}\left(G^{\prime}\right)$ und mit $\eta_{i}$ die identische Abbildung von $G_{i}$ in $R$. Die Abbildung $f \circ \eta_{i}$ ist eine Lindelöfsche Abbildung, da eine, und somit alle Komponenten von $G^{\prime}$ Greensche Funtionen besitzen. Nach Hilfssatz $19^{\prime}$ ist $\hat{f}$ fast überall auf $\Delta_{1}\left(G_{i}\right)$ definiert und, da

$$
\Delta_{1}\left(f^{-1}\left(G^{\prime}\right)\right)=\bigcup_{i} \Delta_{1}\left(G_{i}\right)
$$

ist, so ist $\hat{f}$ auch fast überall auf $\Delta_{1}\left(f^{-1}\left(G^{\prime}\right)\right)$ definiert. Es ist aber $\Delta_{1}-\Delta_{1}\left(f^{-1}\left(G^{\prime}\right)\right)$ vom harmonischen Masse Null und somit ist $\hat{f}$ fast überall auf $\Delta_{1}$ definiert.

Es sei, umgekehrt, $\hat{f}$ fast überall auf $\Delta_{1}$ definiert, $p^{\prime}, q^{\prime} \in R^{\prime}, v_{p^{\prime} q^{\prime}}$ die im Beweis des Satzes 18 eingeführte Funktion und

$$
G_{\alpha}^{\prime}=\left\{r^{\prime} \in R^{\prime} \mid v_{p q^{\prime}}\left(r^{\prime}\right)>\alpha\right\} .
$$

Wir wählen $\alpha$ so, dass $G_{\alpha}^{\prime}$ relativkompakt und

$$
\chi_{R}\left(\hat{f}^{-1}\left(\partial G_{a}^{\prime}\right)\right)=0
$$

sei, was immer möglich ist, und bezeichnen $G^{\prime}=R^{\prime}-\partial G_{\alpha}^{\prime}$. Es sei $s \in \mathfrak{F}(f)-\hat{f}^{-1}\left(\partial G_{\alpha}^{\prime}\right)$. Dann ist entweder $\hat{f}(s) \in G_{\alpha}^{\prime}$, oder $\hat{f}(s) \in \hat{R}^{\prime}-G_{\alpha}^{\prime}$. In beiden Fällen ist $s \in \Delta_{1}\left(f^{-1}\left(G^{\prime}\right)\right)$, woraus

$$
\widetilde{\mho}(f)-\hat{f}^{-1}\left(\partial G_{\alpha}^{\prime}\right) \subset \Delta_{1}\left(f^{-1}\left(G^{\prime}\right)\right)
$$

und

$$
\underset{f^{-1}\left(G^{\prime}\right) f^{-1}\left(G^{\prime}\right)}{I} 1=\omega\left(\Delta_{1}\left(f^{-1}\left(G^{\prime}\right)\right)\right)=1
$$

folgt. $f$ ist also eine Fatousche Abbildung.

Es sei $\left\{a_{n}\right\}$ eine Folge in $|z|<1$, die gegen $|z|=1$ strebt, $\gamma_{n}$ der Kreis mit dem Mittelpunkt in $a_{n}$ und Radius $r_{n}$; wir wählen $r_{n}$ so klein dass

a) $\bar{\gamma}_{n} \subset\{|z|<1\}$,

b) $\bar{\gamma}_{n} \cap \bar{\gamma}_{m}=\phi \quad$ für $n \neq m$

ist. Wir bezeichnen

$$
P_{a b}(z)=\frac{z-a}{1-z \bar{a}} \cdot \frac{1-z \bar{b}}{z-b} .
$$

Auf $|z|=1$ ist $\left|P_{a b}(z)\right|=1$. Aus

$$
\left|\frac{z-a}{1-z \bar{a}}\right|^{2}=1-\frac{\left(1-|z|^{2}\right)\left(1-|a|^{2}\right)}{|1-z a|^{2}}
$$

folgt 


$$
\begin{aligned}
\left|P_{a b}(z)\right|^{2} & =\frac{1-\frac{\left(1-|z|^{2}\right)\left(1-|a|^{2}\right)}{|1-z \bar{a}|^{2}}}{1-\frac{\left(1-|z|^{2}\right)\left(1-|b|^{2}\right)}{|1-z \bar{b}|^{2}}}=1+\frac{\frac{1-|b|^{2}}{|1-z \bar{b}|^{2}}-\frac{1-|a|^{2}}{|1-z \bar{a}|^{2}}}{\left|\frac{z-b}{1-z \bar{b}}\right|^{2}}\left(1-|z|^{2}\right) \\
& =1+(1-|z|) \varepsilon_{a b}(z)
\end{aligned}
$$

mit

$$
\begin{gathered}
\varepsilon_{a b}(z)=\frac{\frac{1-|b|^{2}}{|1-z \bar{b}|^{2}}-\frac{1-|a|^{2}}{|1-z \bar{a}|^{2}}}{\left|\frac{z-b}{1-z \bar{b}}\right|^{2}}(1+|z|) \\
=\frac{\left(1+|z|^{2}\right)\left(|a|^{2}-|b|^{2}\right)+(z+\bar{z} a b)(\bar{b}-\bar{a})+(\bar{z}+z \bar{a} \bar{b})(b-a)}{|z-b|^{2}|1-z \bar{a}|^{2}}(1+|z|) .
\end{gathered}
$$

Es sei $\gamma_{n}^{\prime}$ der Kreis mit dem Mittelpunkt in $a_{n}$ und Radius $r_{n} / 2$. Für $b \in \gamma_{n}^{\prime}$ ist

$$
\varepsilon_{n}(b)=\sup \left\{\left|\varepsilon_{a_{n} b}(z)\right| \mid z \in R-\gamma_{n}\right\} \leq \frac{64\left|b-a_{n}\right|}{\gamma_{n}^{2}\left(1-\left|a_{n}\right|\right)^{2}} .
$$

Wir wählen den Punkt $b_{n}$ so nahe dem Punkte $a_{n}$, dass $b_{n} \in \gamma_{n}^{\prime}$ und

$$
\frac{64\left|b_{n}-a_{n}\right|}{r_{n}^{2}\left(1-\left|a_{n}\right|\right)^{2}}<\frac{1}{2^{n}}
$$

ist und bezeichnen

$$
\begin{aligned}
& f_{n}(z)=z \prod_{j=1}^{n} P_{a_{j} b_{j}}(z), \\
& f(z)=\lim _{n \rightarrow \infty} f_{n}(z)=z \prod_{j=1}^{\infty} P_{a_{j} b_{j}}(z) .
\end{aligned}
$$

Auf $|z|=1$ ist $\left|f_{n}(z)\right|=1$. Auf $\partial \gamma_{j}(j=1,2, \ldots, n)$ ist

$$
\begin{aligned}
& \left|f_{n}(z)\right| \leq|z| \prod_{j=1}^{\infty}\left(1+\frac{1-|z|}{2^{j}}\right) \leq|z| e^{1-|z|}<1, \\
& \left|f_{n}(z)\right| \geq|z| \prod_{j=1}^{\infty}\left(1-\frac{1-|z|}{2^{j}}\right) \geq|z| e^{-2(1-|z|)} .
\end{aligned}
$$

Daraus folgt, dass $f$ eine nichtkonstante meromorphe Funktion ist, die in $G=R-\bigcup_{n=1}^{\infty} r_{n}$ ein kleineres Modul als 1 hat. Es ist also

$$
f^{-1}(\{|w|<1\} \supset G
$$

Wir können immer die Radien $r_{n}$ so klein wählen, dass

c)

$$
\underset{G G}{E I} 1=1
$$


ist. Daraus folgt sofort, dass $f$ eine Fatousche Abbildung ist. Ihre Nullstellen sind gerade die Punkte $a_{n}$ und man kann sie so wählen, dass

$$
\sum_{n=1}^{\infty}\left(1-\left|a_{n}\right|\right)=\infty
$$

ist ; $f$ ist dann keine Lindelöfsche Abbildung. Nimmt man noch die Folge $\left\{a_{n}\right\}$ so, dass sie gegen den Punkt $z=1$ konvergent ist, so kann man zeigen, dass $f$, bis auf den Punkt $z=1$, sogar analytisch auf $|z|=1$ ist (somit ist auch $f^{*}$ fast überall auf $|z|=1$ definiert) und $f$ auch weiter nicht Lindelöfsche Abbildung bleibt.

Wenn wir noch eine Bedingung der Folge $\left\{a_{n}\right\}$ und den Kreisen $r_{n}$ zuschreiben, so erhalten wir ein Beispiel, woraus man ersehen wird, dass die Menge $\mathfrak{F}(f)-\mathfrak{F}^{*}(f)$ in Falle $R^{\prime}=\{|w| \leq \infty\}$ nicht immer leer ist (siehe Seite 64 und den Anhang). Zwar werden wir verlangen, dass

e) die Folge $\left\{a_{n}\right\}$ in allen Punkten $e^{i \theta}$ einen Winkeleingang hat

f) der hyperbolische Durchmesser von $\gamma_{n}$ kleiner als 1 sei. Nach der Bemerkung, die dem Hilfssatz 15 folgt, hat dann auch die Menge $\left\{b_{n}\right\}$ in allen Punkten $e^{i \theta}$ einen Winkeleingang. Daraus ergibt sich da $a_{n}$ die Nullstellen und $b_{n}$ die Pole von $f$ sind, dass die Menge $\widetilde{\vartheta}^{*}(f)$ leer ist, wogegen $\widetilde{F}(f)$ vom Lebesgueschen Masse $2 \pi$ ist.

HiLfSSATz 20. Es sei $R \stackrel{f}{\longrightarrow} R^{\prime} \stackrel{f^{\prime}}{\longrightarrow} R^{\prime \prime}$. a) $\widehat{f^{\circ} \circ f}$ ist auf $\hat{f}^{-1}\left(R^{\prime}\right)$ definiert und gleich $\left.f^{\prime} \circ \hat{f} . \quad b\right) \widehat{f^{\prime} \circ f}$ ist fast überall auf $\hat{f}^{-1}\left(\mathfrak{F}\left(f^{\prime}\right)\right)\left(R^{\prime} \notin O_{G}\right)$ definiert und gleich $\hat{f}^{\prime} \circ \hat{f}$.

Wir setzen $f^{\prime \prime}=f^{\prime} \circ f$.

a) Es sei $s \in \hat{f}^{-1}\left(R^{\prime}\right)$ und $p^{\prime \prime}=f^{\prime}(\hat{f}(s)) . f^{\prime-1}\left(G^{\prime \prime}\left(p^{\prime \prime}, \varepsilon\right)\right)$ ist eine Umgebung von $\hat{f}(s)$ und deshalb ist

$$
\underset{f^{\prime \prime-1}\left(G^{\prime \prime}\left(p^{\prime \prime}, \varepsilon\right)\right)}{I} K_{s}=\underset{f^{-1}\left(f^{\prime-1}\left(G^{\prime \prime}\left(p^{\prime \prime}, \varepsilon\right)\right)\right)}{I} K_{s} \neq 0
$$

Daraus folgt, dass $\hat{f}^{\prime \prime}$ in $s$ definiert und gleich $f^{\prime} \circ \hat{f}(s)$ ist.

b) Es sei $s^{\prime} \in \mathfrak{F}\left(f^{\prime}\right)$. Da

$$
\underset{G^{\prime}\left(S^{\prime}, 1 / n\right)}{I} K_{s^{\prime}}^{\prime} \neq 0, \underset{f^{\prime-1}\left(G^{\prime \prime}\left(\hat{f}^{\prime}\left(s^{\prime}\right), 1 / n\right)\right)}{I} K_{S^{\prime}}^{\prime} \neq 0
$$

ist, so ist laut des Folgesatzes 2 


$$
\underset{G^{\prime} n}{I} K_{s^{\prime}}^{\prime} \neq 0
$$

mit

$$
G_{n}^{\prime}=G^{\prime}\left(s^{\prime}, 1 / n\right) \cap f^{-1}\left(G^{\prime \prime}\left(\hat{f}^{\prime}\left(s^{\prime}\right), 1 / n\right)\right) .
$$

Ist ausserdem $s^{\prime}$ in $\Delta_{1}^{\prime}(f)$ enthalten, so folgt aus dem Hilfssatz 6

$$
\underset{f^{-1}\left(G n^{\prime}\right)}{E} \underset{f^{-1}\left(G n^{\prime}\right)}{I} I_{f} K_{S^{\prime}}^{\prime}=I_{f} K_{s^{\prime}}^{\prime}
$$

Daraus und aus den Sätzen 14 und $15^{\prime}$ erhalten wir

$$
I_{f} K_{s^{\prime}}^{\prime}=\int_{\Delta_{1}(f) \cap \Delta_{1}\left(f^{-1}\left(G_{n^{\prime}}\right)\right)} K_{s} d\left(\delta_{s^{\prime}} \circ f\right)(s) .
$$

Lassen wir $n$ gegen unendlich streben so ergibt sich

$$
I_{f} K_{S^{\prime}}^{\prime}=\int_{\Delta_{1}(f) \cap \underset{n=1}{\infty} \Delta_{1}^{\infty}\left(f^{-1}\left(G_{n^{\prime}}\right)\right)} K_{s} d\left(\delta_{s^{\prime}} \circ f\right)(s) .
$$

Wir bezeichnen mit $A$ die Menge

$$
A=\left\{s \in \hat{f}^{-1}\left(\mathfrak{F}\left(f^{\prime}\right)\right) \mid s \in \mathfrak{F}\left(f^{\prime \prime}\right), \quad \hat{f}^{\prime \prime}(s)=\hat{f}^{\prime}(\hat{f}(s))\right\} .
$$

$A$ ist offenbar eine Borelsche Menge: Man erkennt sofort, dass

$$
\Delta_{1}(f) \cap \bigcap_{n=1}^{\infty} \Delta_{1}\left(f^{-1}\left(G_{n}^{\prime}\right)\right) \subset A \subset \Delta_{1}(f)
$$

ist. Es ist also

$$
I_{f} K_{s^{\prime}}^{\prime}=\int_{A} K_{s} d\left(\delta_{s^{\prime}} \circ f\right)(s)
$$

und aus dem Satz 14 folgt

$$
\begin{aligned}
I_{f} \omega & \left(\widetilde{F}\left(f^{\prime}\right), R^{\prime}\right)=I_{f} \int_{\mathscr{F}\left(f^{\prime}\right)} K_{s^{\prime}}^{\prime} d \chi_{R^{\prime}}\left(s^{\prime}\right)=I_{f} \int_{\mathscr{F}_{\left(f^{\prime} ; \cap \Delta_{1^{\prime}}(f)\right.}} K_{s^{\prime}}^{\prime} d \chi_{R^{\prime}}\left(s^{\prime}\right) \\
& =\int_{A} K_{s} d\left(\chi_{R^{\prime}} \circ f\right)(s)=\int_{A} K_{s} d \chi_{R}(s)=\omega(A, R) .
\end{aligned}
$$

Laut des Satzes 17 ist aber

$$
I_{f} \omega\left(\widetilde{\mathfrak{F}}\left(f^{\prime}\right), R^{\prime}\right)=\omega\left(\hat{f}^{-1}\left(\widetilde{\jmath}\left(f^{\prime}\right)\right), R\right)
$$

und somit ist

$$
\hat{f}^{-1}\left(\widetilde{F}\left(f^{\prime}\right)\right)=A:\left[\%_{R}\right]
$$

was die Behauptung von b) bestätigt.

SAtz 25. Es sei $R \stackrel{f}{\longrightarrow} R^{\prime} \stackrel{f^{\prime}}{\rightarrow} R^{\prime \prime}$ und $f$ eine Fatousche Abbildung. Ist $R^{\prime} \notin O_{G}$ 
und $f^{\prime}$ eine Fatousche Abbildung oder ist $R^{\prime}$ beliebig und $\Delta_{1}(f)$ vom harmonischen Masse Null (was immer der Fall ist, wenn $R^{\prime} \in O_{G}$ ist), so ist $f^{\prime} \circ f$ eine Fatousche Abbildung.

Laut des Hilfssatzes 20 ist

$$
\hat{f}^{-1}\left(R^{\prime} \cup \mathfrak{F}\left(f^{\prime}\right)\right) \subset \mathfrak{F}\left(f^{\prime} \circ f\right):\left[\chi_{R}\right] .
$$

Ist $R^{\prime} \notin O_{G}$ und $f^{\prime}$ eine Fatousche Abbildung, so ist $\Delta^{\prime}-\mathfrak{F}\left(f^{\prime}\right)$ eine polare Menge und $\hat{f}^{-1}\left(\Delta^{\prime}-\mathfrak{F}\left(f^{\prime}\right)\right)$ eine Menge vom harmonischen Masse Null. Es ist also

$$
1 \geq \chi_{R}\left(\mathfrak{f}\left(f^{\prime} \circ f\right)\right) \geq \chi_{R}\left(\hat{f}^{-1}\left(R^{\prime} \cup \mathfrak{F}\left(f^{\prime}\right)\right)\right)=\chi_{R}\left(\hat{f}^{-1}\left(R^{\prime} \cup \Delta^{\prime}\right)\right)=\chi_{R}(\mathfrak{F}(f))=1
$$

und $f^{\prime} \circ f$ ist eine Fatousche Abbildung. Ist $\Delta_{1}(f)$ vom harmonischen Masse Null, so ist

$$
1 \geq \chi_{R}\left(\mathfrak{F}\left(f^{\prime} \circ f\right)\right) \geq \chi_{R}\left(\hat{f}^{-1}\left(R^{\prime}\right)\right)=\chi_{k}\left(\hat{f}^{-1}\left(R^{\prime} \cup \Delta^{\prime}\right)\right)=\chi_{R}(\mathfrak{F}(f))=1
$$

und $f^{\prime} \circ f$ ist eine Fatousche Abbildung.

SATZ 26. Es gibt eine Menge $Z(f) \subset \Delta_{1}$, vom harmonischen Masse Null, derart dass für jedes $s \in \Delta_{1}-Z(f)-\mathfrak{\wp}(f)$

$$
\hat{M}(s)=\hat{R}^{\prime}
$$

ist, und für jede Umgebung $\hat{G}$ von $s$ ist $R^{\prime}-f(\hat{G} \cap R)$ eine Menge der Kapazität Null.

Es sei $\left\{G_{i}^{\prime}\right\}$ eine Folge von Kreisscheiben auf $R^{\prime}$ die eine Basis in $R^{\prime}$ bilden. Die Mengen $f^{-1}\left(R^{\prime}-\bar{G}_{i}^{\prime}\right)$ sind offen; wir bezeichnen mit $\left\{G_{n}\right\}$ die Komponenten aller dieser Mengen $(i=1,2, \ldots)$. Ist $\hat{M}(s) \neq \hat{R}^{\prime}$, so gibt es ein $G_{i}^{\prime}$, für welches

$$
\hat{M}(s) \subset \hat{R}^{\prime}-\bar{G}_{i}^{\prime}
$$

und es ist $s \in \Delta_{1}\left(G_{n}\right)$ für ein $n$. Es ist also für $s \notin \bigcup_{n=1}^{\infty} \Delta_{1}\left(G_{n}\right)$

$$
\hat{M}(s)=\hat{R}^{\prime} \text {. }
$$

Wir bezeichnen

$$
Z_{1}=\bigcup_{n=1}^{\infty} \Delta_{1}\left(G_{n}\right)-\widetilde{F}(f)
$$

Laut des Hilfssatzes $19^{\prime}$ ist $\Delta_{1}\left(G_{n}\right)-\mathfrak{F}(f)$ und somit auch $Z_{1}$ vom harmonischen Masse Null.

Es sei jetzt $\left\{\hat{D}_{i}\right\}$ eine abzählbare Basis in $\hat{R}$ und $\mathfrak{N}$ die Menge der Zahlen 
$i$, für welche $R^{\prime}-f\left(\hat{D}_{i} \cap R\right)$ eine Menge mit positiver Kapazität ist. Es seien $\left\{G_{n}\right\}$ die Komponenten der Menge $\hat{D}_{i} \cap R$ für $i \in \mathfrak{N}$ und

$$
Z_{2}=\bigcup_{n} \Delta_{1}\left(G_{n}\right)-\mathfrak{F}(f) .
$$

Nach dem Hilfssatz $19^{\prime}$ folgt, dass $\Delta_{1}\left(G_{n}\right)-\mathfrak{F}(f)$ und somit $Z_{2}$ vom harmonischen Masse Null sind. Es sei $s \in \Delta_{1}-\cup \Delta_{1}\left(G_{n}\right)$ und $\hat{G}$ eine Umgebung von $s$. Dann gibt es ein $\hat{D}_{i}$ für welches $s \in \hat{D}_{i} \subset \hat{G}$ ist. Wäre $i \in \Re$, so folgt aus $s \in \Delta_{1}\left(\hat{D}_{i} \cap R\right)$, dass für wenigstens eine Komponente $G_{n}$ von $\hat{D}_{i} \cap R, s \in \Delta_{1}\left(G_{n}\right)$ ist, was der Voraussetzung über $s$ widerspricht. Es ist also

$$
R^{\prime}-f(\hat{G} \cap R) \subset R^{\prime}-f\left(\hat{D}_{i} \cap R\right),
$$

woraus man erkennt, dass $R^{\prime}-f(\hat{G} \cap R)$ eine Menge der Kapazität Null ist. Der Satz folgt sofort aus

$$
Z(f)=Z_{1} \cup Z_{2}
$$

\section{Abbildungen vom Typus Bl}

HilfsSatz 21. Es sei $S \in S P(R)$ und $v$ die grösste quasibeschränkte Minorante von $S$. Setzt man

$$
\begin{aligned}
& v=\int_{\Delta_{1}} K_{s} \theta(s) d \chi(s), \\
& A_{\alpha}=\left\{s \in \Delta_{1} \mid \theta(s)>\alpha\right\}, \\
& B_{\alpha}=\left\{s \in \Delta_{1} \mid \theta(s) \geq \alpha\right\}, \\
& G_{\alpha}=\{p \in R \mid S(p)>\alpha\},
\end{aligned}
$$

so ist

$$
A_{\alpha} \subset \Delta_{1}\left(G_{\alpha}\right) \subset B_{\alpha}:[\%] .
$$

Ist $S$ stetig (unendlich nicht ausgeschlossen), so ist auch

$$
\Delta_{1}-B_{\alpha} \subset \Delta_{1}\left(R-\bar{G}_{\alpha}\right):[\chi] \text {. }
$$

Da $D_{\alpha}=\{p \in R \mid v(p)>\alpha\} \subset G_{\alpha}$ ist, so ist $\Delta_{1}\left(D_{\alpha}\right) \subset \Delta_{1}\left(G_{\alpha}\right)$. Nach Hilfssatz 11 ist aber $A_{\alpha} \subset A_{1}\left(D_{\alpha}\right):[\chi]$, woraus $A_{\alpha} \subset \Delta_{1}\left(G_{\alpha}\right):[\chi]$ folgt. Auf $G_{\alpha}$ ist

$$
\underset{\sigma_{\alpha}}{I} 1 \leq 1 \leq \frac{1}{\alpha} S
$$

und somit ist 


$$
\underset{\sigma_{\alpha} \alpha \alpha}{E} I 1 \leq 1 \leq \frac{1}{\alpha} S
$$

auf $R$. Indem man die Definition von $v$ in Betracht zieht, ergibt sich

$$
\underset{\sigma_{\alpha} G_{\alpha}}{E I} 1 \leq \frac{1}{\alpha} v=\int_{\Delta_{1}} K_{s} \frac{\theta(s)}{\alpha} d \chi(s) .
$$

Nach Satz $15^{\prime}$ ist

$$
\underset{G \alpha G \alpha}{E I} 1=\int_{\Delta_{1}(G \alpha)} K_{s} d \chi(s)
$$

woraus, mittels des Hilfssatzes a, $\psi \leq \frac{\theta}{\alpha}$ folgt, wo $\psi$ die Charakteristische Funktion der Menge $\Delta_{1}\left(G_{\alpha}\right)$ ist. $\theta$ ist also auf $\Delta_{1}\left(G_{\alpha}\right)$ nicht kleiner als $\alpha$, bis auf eine Menge vom harmonischen Masse Null, was äquivalent mit $\Delta_{1}\left(G_{\alpha}\right) \subset B_{\alpha}:[\%]$ ist.

Wir bezeichnen $D=R-\bar{G}_{\alpha}$. Laut des Hilfssatzes 4 ist

$$
\alpha=\underset{D}{I} \alpha+H_{D}^{\alpha} \leq I_{D} \alpha+S \leq \underset{D D}{E I} \alpha+S
$$

auf $D$, denn $S \in \overline{\mathfrak{B}}_{D}^{\alpha}$. Auf $R-D$ ist

$$
\alpha \leq \underset{D D}{E I} \alpha+S
$$

offenbar gültig. Daraus folgt

$$
\alpha-\underset{D}{E} I_{D} \alpha \leq v
$$

und nach Satz $15^{\prime}$

$$
\alpha \int_{\Delta_{1}-\Delta_{1}(D)} K_{\varsigma} d \chi(s) \leq \int_{\Delta_{1}} K_{s} \theta(s) d \chi(s) .
$$

Es ist somit $\alpha \leq \theta(s):[\chi]$ auf $\Delta_{1}-\Delta_{1}(D)$ und deshalb

$$
\Delta_{1}-B_{\alpha} \subset \Delta_{1}(D):[\chi]
$$

was $\mathrm{zu}$ beweisen war.

Hilfssatz 22. Es ser $P^{\prime}$ ein Potenzial auf $R^{\prime}\left(R^{\prime} \notin O_{G}\right)$, u die grösste harmonische Minorante von $P^{\prime} \circ f$ und $v$ die quasibeschränkte Komponente von $u$ :

$$
u=\int_{\Delta_{1}} K_{s} d \mu(s), \quad v=\int_{\Delta_{1}} K_{s} \theta(s) d \chi(s) .
$$

Es ist

$$
\mu\left(\Delta_{1}(f)\right)=0,
$$




$$
\left\{s \in \Delta_{1} \mid \theta(s)=0\right\}=\Delta_{1}(f):[\%]
$$

Es sei

$$
u_{0}=\int_{\Delta_{1}(f)} K_{s} d \mu(s)
$$

Nach Hilfssatz 7 ist $u_{0} \in H P(f)$ und $E u_{0}$ ist harmonisch. Da aber $E u_{0}$ nicht grösser als das Potenzial $P^{\prime}$ ist, so sind $E u_{0}$ und $u_{0}$ Null und $\mu_{(}\left(\Delta_{1}(f)\right)=0$. Daraus folgt

$$
\left\{s \in \Delta_{1} \mid \theta(s)=0\right\} \supset \Delta_{1}(f):[\chi] .
$$

Wir bezeichnen

$$
\begin{aligned}
& G_{\alpha}^{\prime}=\left\{p^{\prime} \in R^{\prime} \mid P^{\prime}\left(p^{\prime}\right)>\alpha\right\}, \\
& G_{\alpha}=f^{-1}\left(G_{a}^{\prime}\right)=\left\{p \in R \mid P^{\prime} \circ f(p)>\alpha\right\} .
\end{aligned}
$$

Nach der Eigenschaft c! von $\hat{f}$ ist

$$
\left(\Delta_{1}-\Delta_{1}(f)\right) \cap \widetilde{F}(f) \subset \bigcup_{\alpha>0} \Delta_{1}\left(G_{\alpha}\right)
$$

und nach Hilfssatz 21 ist

$$
\Delta_{1}\left(G_{\alpha}\right) \subset\left\{s \in \Delta_{1} \mid \theta(s) \geq \alpha\right\}:[\chi] .
$$

Daraus folgt

$$
\left\{s \in \Delta_{1} \mid \theta(s)=0\right\} \subset \Delta_{1}(f):[\chi],
$$

was den Beweis beendigt.

Für $R^{\prime} \notin O_{G}$ bezeichnet Heins [5] mit $u_{p^{\prime}}$ die grösste harmonische Minorante von $g_{p^{\prime}}^{\prime} \circ f$ und mit $v_{p^{\prime}}$, bzw. $w_{p^{\prime}}$, die quasibeschränkte, bzw. singuläre, Komponente von $u_{p^{\prime}}$. Die Abbildung $f$ heisst vom Typus $\mathrm{Bl}$, bzw. $\mathrm{Bl}_{1}$, wenn $v_{p^{\prime}}$, bzw. $u_{p^{\prime}}$, verschwindet für alle $p^{\prime} \cdot{ }^{17)}$

SATz 27. $f$ ist vom Typus Bl dann und nur dann, wenn

$$
\Delta_{1}(f)=\Delta_{1}:[\chi]
$$

ist. Diese Bedingung ist gleichbedeutend mit

$$
\chi\left(\hat{f}^{-1}\left(R^{\prime}\right)\right)=0 .
$$

Ist $\Delta_{1}(f)=\Delta_{1}$, so ist $f$ vom Typus $B l_{1}$.

Da $g_{p^{\prime}}^{\prime}$ ein Potenzial auf $R^{\prime}$ ist, so folgt aus dem Hilfssatz 22, dass $v_{p^{\prime}}=0$

17) Sind $R$ und $R^{\prime}$ die Kreise $|z|<1$ und $|w|<1$, so folgt daraus, dass die Abbildungen vom Typus $\mathrm{Bl}$ gerade dịe Seịdęlschen Funḳtionen sind. 
und $\Delta_{1}(f)=\Delta_{1}:[\chi]$ äquivalente Beziehungen sind, woraus die erste Behauptung folgt. Ist $\Delta_{1}(f)=\Delta_{1}$, so haben wir aus demselben Hilfssatz $u_{p^{\prime}}=0$.

Für ein beliebiges $R^{\prime}$ und einen Punkt $p^{\prime} \in R^{\prime}$ heisst $f$ lokal vom Typus $\mathrm{Bl}$, bzw. $\mathrm{Bl}_{1}$, im Punkte $\not^{\prime}$, wenn man eine zusammenhängende Umgebung mit Greenscher Funktion $G^{\prime}$ von $p^{\prime}$ finden kann, für die alle Abbildungen der Komponenten von $f^{-1}\left(G^{\prime}\right)$ in $G^{\prime}$, die mit $f$ zusammenfallen, vom Typus Bl, bzw. $\mathrm{Bl}_{1}$, sind. Matsumoto [12] hat gezeigt, dass $f$ im Punkte $p^{\prime}$ lokal vom Typus $\mathrm{Bl}$ ist, dann und nur dann, wenn man ein Jordansches Gebiet $G^{\prime}$ finden kann, das $p^{\prime}$ enthält und für welches alle Komponenten von $f^{-1}\left(G^{\prime}\right)$ vom Typus $S O_{B B}$ sind. Für eine offene Menge $G$ auf $R$ sind alle Komponenten vom Typus $S O_{H B}$ dann und nur dann, wenn $\Delta_{1}(G)$ vom harmonischen Masse Null ist. Daraus folgt, dass $f$ im Punkie $p^{\prime}$ dann und nur dann lokal vom Typus $B l$ ist, wenn man eine Umgebung $G^{\prime}$ von $p^{\prime}$ finden kann, für die $\hat{f}^{-1}\left(G^{\prime}\right)$ vom harmonischen Masse Null ist.

Wir nehmen an, dass $R^{\prime} \notin O_{G}$ ist und setzen

$$
\begin{aligned}
& V_{f}=\left\{p^{\prime} \in R^{\prime} \mid v_{p^{\prime}} \text { nicht beschränkt }\right\} \\
& W_{f}=\left\{p^{\prime} \in R^{\prime} \mid w_{p^{\prime}} \neq 0\right\} .
\end{aligned}
$$

$W_{f}$ ist, wie Heins bewiesen hat [5], eine Menge vom Typus $F_{\sigma}$ und der Kapazität Null. $V_{f}$ ist gerade die Menge wo $f$ nicht lokal vom Typus $B l$ ist.

In der Tat ist $\phi^{\prime}$ nicht in $V_{f}$ enthalten, so ist $v_{p^{\prime}}<\alpha<\infty$ für eine bestimmte positive Zahl $\alpha$. Wir bezeichnen

$$
\begin{aligned}
& v_{p^{\prime}}=\int_{\Delta_{1}} K_{s} \theta_{p^{\prime}}(s) d \chi(s), \\
& G_{\alpha}^{\prime}=\left\{q^{\prime} \in R^{\prime} \mid g_{p^{\prime}}\left(q^{\prime}\right)>\alpha\right\}, \quad G_{\alpha}=f^{-1}\left(G_{\alpha}^{\prime}\right) .
\end{aligned}
$$

Nach Hilfssatz 21 ist

$$
\hat{f}^{-1}\left(G_{\alpha}^{\prime}\right) \subset \Delta_{1}\left(G_{\alpha}\right) \subset\left\{s \in \Delta_{1} \mid \theta_{p^{\prime}}(s) \geq \alpha\right\}=\phi:[\chi]
$$

und $f$ ist lokal vom Typus $\mathrm{Bl}$ im Punkte $p^{\prime}$. Umgekehrt, ist $f$ lokal vom Typus $\mathrm{Bl}$ im Punkte $p^{\prime}$, so ist $\hat{f}^{-1}\left(G^{\prime}\right)$ vom harmonischen Masse Null für eine Umgebung $G^{\prime}$ von $p^{\prime}$. Für $\alpha$ genugend gross ist $G_{\alpha}^{\prime} \subset G^{\prime}$ und nach Hilfssatz 21 ist

$$
\left\{s \in \Delta_{1} \mid \theta_{p^{\prime}}(s)>\alpha\right\} \subset \Delta_{1}\left(G_{\alpha}\right) \subset \Delta_{1}\left(\hat{f}^{-1}\left(G^{\prime}\right)\right):[\chi],
$$

woraus $v_{p^{\prime}} \leq \alpha$ und $p^{\prime} \notin V_{f}$ folgt. 
Ist $p^{\prime} \in W_{f}$, so ist $\hat{f}^{-1}\left(p^{\prime}\right)$ nicht leer und $w_{p^{\prime}}$ ist in der Form

$$
w_{p^{\prime}}=\int_{\hat{f}^{-1}\left(p^{\prime}\right)} K ; d \mu(s)
$$

darstellbar. Wir setzen

$$
D_{\alpha}=\left\{p \in R \mid w_{p},(p)>\alpha\right\} \subset G_{\alpha} .
$$

Die Behauptungen folgen unmittelbar aus den Gleichheiten

$$
\begin{aligned}
& w_{p^{\prime}}=\underset{D_{\alpha} D_{\alpha}}{E} w_{p^{\prime}}=\int_{\Delta_{1}\left(D_{\alpha}\right)} K_{s} d \mu(s)=\int_{\Delta_{1}(G \alpha)} K_{s} d \mu(s), \\
& \hat{f}^{-1}\left(p^{\prime}\right)=\bigcap_{\alpha} \Delta_{1}\left(G_{\alpha}\right) .
\end{aligned}
$$

Daraus folgt [5], dass $p^{\prime}$ ein asymptotischer Punkt ist.

SATz 28. Es sei $u \in H P(R)$ und $E u=u^{\prime}+P^{\prime} \neq \infty$, wo $u^{\prime} \in H P\left(R^{\prime}\right)$ und $P^{\prime}$ ein Potential ist. Dann ist

$$
P^{\prime}=\int_{V_{f} \cup W_{f}} g_{p^{\prime}}^{\prime} d \mu^{\prime}\left(p^{\prime}\right)
$$

Ist $u$ quasibeschränkt, so ist

$$
\mu\left(W_{f}-V_{f}\right)=0
$$

ist u singulär, so ist

$$
\mu\left(V_{f}-W_{f}\right)=0 .
$$

In diesem letzten Falle ist $u^{\prime}$ singulär. ${ }^{18)}$

Es sei

$$
P^{\prime}=\int_{R^{\prime}} g_{p^{\prime}}^{\prime} d \mu^{\prime}\left(p^{\prime}\right)
$$

und

$$
P^{\prime \prime}=\int_{R^{\prime}-V_{f} \cup W_{f}} g_{p^{\prime}}^{\prime} d \mu^{\prime}\left(p^{\prime}\right) .
$$

Dann ist $E u-P^{\prime \prime} \in S P\left(R^{\prime}\right)$ und $u \leq P^{\prime \prime} \circ f+\left(E u-P^{\prime \prime}\right) \circ f$. Nach dem Kjellbergschen Hilfssatz kann man zwei harmonische Funktionen $u_{1}, u_{2}$ finden, so dass

$$
\begin{aligned}
& u=u_{1}+u_{2}, \\
& 0 \leq u_{1} \leq P^{\prime \prime} \circ f, \quad 0 \leq u_{2} \leq\left(E u-P^{\prime \prime}\right) \circ f
\end{aligned}
$$

18) Aus $u$ quasibeschränkt folgt nicht immer $u^{\prime}$ quasibeschränkt. 
ist. Es ist aber

$$
P^{\prime \prime} \circ f=\int_{R^{\prime}-r_{f} \cup W_{f}}\left(g_{p^{\prime}}^{\prime} \circ f\right) d \mu^{\prime}\left(p^{\prime}\right) .
$$

Heins hat bewiesen [5], dass die grösste harmonische Minorante von $P^{\prime \prime} \circ f$ gleich

$$
\int_{R^{\prime}-V_{f} \cup w_{f}} u_{p^{\prime}} d \mu^{\prime}\left(p^{\prime}\right)
$$

ist. Es ist somit

$$
u_{1} \leq \int_{R^{\prime}-\mathrm{v}_{f} \cup W_{f}} u_{p^{\prime}} d \mu^{\prime}\left(p^{\prime}\right)=\int_{R^{\prime}-V^{\prime} f W_{f}} v_{p^{\prime}} d \mu^{\prime}\left(p^{\prime}\right),
$$

denn $u_{p^{\prime}}=v_{p^{\prime}}$ auf $R^{\prime}-V_{f} \cup W_{f}$. Daraus ersieht man, dass $u_{1}$ quasibeschränkt ist.

Aus

$$
E u=E\left(u_{1}+u_{2}\right) \leq E u_{1}+E u_{2} \leq E u_{1}+E u-P^{\prime \prime} \leq E u
$$

folgt $E u_{1}=P^{\prime \prime}$.

Für einen Punkt $p^{\prime} \notin V_{f}$ kann man eine Umgebung $G^{\prime}$ finden, für welche

$$
\underset{f^{-1}\left(G^{\prime}\right)}{I} 1=0
$$

ist. Daraus folgt

$$
\underset{f^{-1}\left(G^{\prime}\right)}{I}\left(u_{1} \wedge n\right) \leq n \underset{f^{-1}\left(G^{\prime}\right)}{I} 1=0
$$

und

$$
\underset{f^{-1}\left(f^{\prime}\right)}{I} u_{1}=\lim _{n \rightarrow \infty} \underset{f^{-1\left(f^{\prime}\right)}}{I}\left(u_{1} \wedge n\right)=0
$$

Nach Hilfssatz 4 ist

$$
H_{f^{-1}\left(G^{\prime}\right)}^{u_{1}}=u_{1}
$$

und nach Hilfssatz 1 ist $E u_{1}$ harmonisch in $G^{\prime}$. Es ist somit $\mu^{\prime}\left(G^{\prime}\right)=0$ und, da $p^{\prime}$ beliebig war,

$$
\mu^{\prime}\left(R^{\prime}-V_{f}\right)=0, \quad P^{\prime \prime}=0 .
$$

Ist $u$ quasibeschränkt, so folgt genau wie oben für $u_{1}$

$$
\mu^{\prime}\left(W_{f}-V_{f}\right) \leq \mu^{\prime}\left(R^{\prime}-V_{f}\right)=0 .
$$

Ist $u$ singulär, so bezeichnen wir

$$
P^{\prime \prime \prime}=\int_{V_{f}-w_{f}^{\prime}} g_{p^{\prime}}^{\prime} d \mu^{\prime}\left(p^{\prime}\right),
$$


Mittels des Kjellbergschen Hilfssatzes kann man zwei harmonische Funktionen $u_{1}, u_{2}$ finden, derart dass

$$
\begin{aligned}
& u=u_{1}+u_{2}, \\
& 0 \leq u_{1} \leq P^{\prime \prime \prime} \circ f, \quad 0 \leq u_{2} \leq\left(E u-P^{\prime \prime \prime}\right) \circ f
\end{aligned}
$$

ist. $u_{1}$ ist nicht grösser als die grösste harmonische Minorante von $P^{\prime \prime \prime} \circ f$, welche, wie Heins bewiesen hat, gleich

$$
\int_{\mathrm{v}_{f}-w_{f}} u_{p^{\prime}} d \mu^{\prime}\left(p^{\prime}\right)
$$

ist. Hier kann man $v_{p^{\prime}}$ an Stelle von $u_{p^{\prime}}$ setzen, da $w_{p^{\prime}}$ Null auf $V_{f}-W_{f}$ ist. Daraus erkennt man, dass $u_{1}$ quasibeschränkt und somit Null ist. Es ist also auch $P^{\prime \prime \prime}$ Null und

$$
\mu\left(V_{f}-W_{f}\right)=0 .
$$

Es sei $v^{\prime}$ eine nichtnegative beschränkte harmonische Funktion auf $R^{\prime}, v^{\prime} \leq E u$. Mittels des Kjellbergschen Hilfssatzes sieht man genau wie oben, dass $u \leq\left(E u-v^{\prime}\right) \circ f$ ist, woraus $v^{\prime}=0$ folgt.

Wir werden mit $O_{H B_{n}}(1 \leq n \leq \infty)$ die Klasse der Riemannschen Flächen mit Greenscher Funktion bezeichnen, für die $\Delta_{1}$ aus höchstens $n$ Punkten $s_{1}, s_{2}$, $\ldots, s_{n}$, für die $K_{s}$ beschränkt ist, und aus einer Menge vom harmonischen Masse Null besteht [1].

SATz 29. Ist $R \in O_{H B_{\infty}}$, so ist $f$ lokal vom Typus $B l$ [12]. Ist $R \in O_{H B_{n}}$ $-\bigcup_{i<n} O_{H B_{i}}(1 \leq n \leq \infty)$ und $R^{\prime} \notin O_{G}$, so ist $R^{\prime} \in O_{H B_{n^{\prime}}}-\bigcup_{i<n^{\prime}} O_{H B_{i}}$ für ein $n^{\prime}$,

$$
n^{\prime} \leq n \leq n^{\prime} \sup \nu_{f}
$$

Laut des Folgesatzes 3 ist $\hat{f}^{-1}\left(R^{\prime}\right) \subset \Delta_{1}-\bigcup_{i=1}^{n}\left\{s_{i}\right\}$ und somit vom harmonischen Masse Null, woraus sofort folgt, dass $f$ lokal vom Typus Bl ist. Ist $R^{\prime} \notin O_{G}$, so ist nach demselben Folgesatz $\hat{f}$ in allen Punkten $s_{i}$ definiert, $\hat{f}\left(s_{i}\right) \in \Delta_{1}^{\prime}$ und $K_{\hat{f}\left(s_{i}\right)}^{\prime}$ ist beschränkt. Es sei $A^{\prime}=\Delta_{1}^{\prime}-\bigcup_{i=1}^{n}\left\{\hat{f}\left(s_{i}\right)\right\}$. Dann ist nach Satz 17

$$
I \omega\left(A^{\prime}, R^{\prime}\right)=\omega\left(\hat{f}^{-1}\left(A^{\prime}\right), R\right) \leq \omega\left(\Delta_{1}-\bigcup_{i=1}^{n}\left\{s_{i}\right\}, R\right)=0 .
$$

Daraus und aus

$$
I \omega\left(A^{\prime}, R^{\prime}\right)=\omega\left(A^{\prime}, R^{\prime}\right) \circ f
$$


(Satz 27) folgt, dass $A^{\prime}$ vom harmonischen Masse Null ist. Es ist also $R^{\prime} \in O_{H B_{n^{\prime}}}-\bigcup_{i<n} O_{H B_{i}}$ mit $n^{\prime} \leq n$. Wir nehmen jetzt an, dass sup $\nu_{f}<\infty$ ist. Für jedes $s^{\prime} \in \Delta_{1}^{\prime}$ für welches $K_{s^{\prime}}^{\prime}$ beschränkt ist, enthält $\hat{f}^{-1}\left(s^{\prime}\right)$ höchstens sup $\nu_{f}$ Punkte $s_{i}$ (Folgesatz 6) woraus $n \leq n^{\prime} \sup \nu_{f}$ folgt.

\section{LITERATUR}

[1] Constantinescu, C. und Cornea, A., Über den idealen Rand und einige seiner Anwendungen bei der Klassifikation der Riemannschen Flächen, Nagoya Math. J., 13 (1958), 169-233.

[2] Constantinescu, C. et Cornea, A., Comportement des transformations analytiques des surfaces de Riemann sur la frontière de Martin, Comptes Rendus, Paris, 249 (1959), 355-357.

[3] Halmos, P., Measure Theory, D. Van Nostrand Company (1951).

[4] Heins, M., Riemann surfaces of infinite genus, Ann. of Math., 55 (1952), 296-317.

[5] Heins, M., On the Lindelöf principle, Ann. of Math., 61 (1955), 440-473.

[6] Heins, M., Lindelöfian maps, Ann. of Math., 62 (1955), 418-446.

[7] Heins, M., On the principle of harmonic measure, Comm. Math. Helv., 33 (1959), $47-58$.

[8] Kjellberg, B., On the growth of minimal positive harmonic functions in a plane region, Arkiv för Mat., Bd. I, Nr. 25 (1950), 347-351.

[ 9 ] Kuramochi, Z., Relations between harmonic dimensions, Proc. Japan Acad., 30 (1954), 576-580.

[10] Kuramochi, Z., On the ideal boundaries of abstract Riemann surfaces, Osaka Math. J., 10 (1958), 83-102.

[11] Martin, R. S., Minimal positive harmonic functions, Trans. Amer. Math. Soc., 49 (1941), 137-172.

[12] Matsumoto, K., Remarks on some Riemann surfaces, Proc. Japan Acad., 34 (1958), 672-675.

[13] Parreau, M., Sur les moyennes des fonctions harmoniques et analytiques et la classification des surfaces de Riemann, Ann. Inst. Fourier, 3 (1952) 103-197.

[14] Pfluger, A., Theorie der Riemannschen Flächen, Springer Verlag (1957).

[15] Stoïlow, S., Leçons sur les principes topologiques de la théorie des fonctions analytiques, Deuxième édition, Gauthier Villars (1956).

[16] Tsuji, M., Remarks on my former paper "On an extension of Löwner's theorem", Comm. Math. Univ. St. Pauli, 4 (1955) 109-110.

\section{Mathematisches Institut}

Rumänische Akademie 


\section{Anhang*}

Wir geben ein Beispiel einer analytischen Funktion $f$ im Kreise $|z|<1$, für die $\hat{f}$ im Punkte $z=1$ definiert und gleich $\infty$ ist, wogegen $f^{*}$ im Punkte $z=1$ nicht definiert ist. Dieses Beispiel zeigt, dass der Satz 21 auf den ausgeschlossenen Fall $R^{\prime}=\{|w|<\infty\}, \hat{f}\left(e^{i \theta}\right)=\infty$, nicht ausgedehnt werden kann.

Es sei $G$ die rechte Halbebene und $\gamma_{n}$ der Kreis

$$
\left|\zeta-2^{n^{3}}\right| \leq 1, \quad(n=1,2, \ldots)
$$

Es ist

$$
H_{G-r_{n}}^{\xi}(1) \leq\left(2^{n^{3}}+1\right) \omega_{n}(1), \quad(\xi=\operatorname{Re} \zeta)
$$

wo $\omega_{n}$ das harmonische Mass von $\partial \gamma_{n}$ auf $G-\gamma_{n}$ ist. Bezeichnet man mit $a_{n}$ den hyperbolischen Mittelpunkt von $\gamma_{n}$, so ist

$$
\boldsymbol{a}_{n}=\sqrt{2^{2 n^{3}}-1}
$$

$\omega_{n}(\zeta)$ ist $\mathrm{zu} \log \frac{\zeta+a_{n}}{\zeta-a_{n}}$ proportional; daraus folgt

$$
{ }^{\prime}{ }_{n}(1)=\frac{\log \frac{1+a_{n}}{1-a_{n}} \mid}{\log \mid \frac{2^{n^{3}}+1+a_{n}}{2^{n^{3}}+1-a_{n}}} .
$$

Es ist aber

$$
\begin{aligned}
& \log \left|\frac{1+a_{n}}{1-a_{n}}\right|=\log \left|\frac{1+\frac{1}{a_{n}}}{1-\frac{1}{a_{n}}}\right| \leq 2 \frac{\frac{1}{a_{n}}}{1-\frac{1}{a_{n}}} \leq \frac{2}{a_{n-1}} \leq \frac{1}{2^{n_{n}-2}}, \\
& \log \left|\frac{{\frac{n^{3}}{3}}^{3}+1+a_{n}}{2^{n^{3}}+1-a_{n}}\right|=\log \frac{\sqrt{2^{n^{3}}+1}+\sqrt{2^{n^{3}}-1}}{\sqrt{2^{n^{3}}+1}-\sqrt{2^{n^{3}}-1}} \geq \log \frac{2.2^{n^{3}}}{2}=n^{3} \log 2,
\end{aligned}
$$

und somit haben wir

$$
\omega_{n}(1) \leq \frac{1}{n^{3} 2^{n^{3}-2} \log 2}
$$

Daher ergibt sich

$$
H_{G-r_{n}}^{\xi}(1) \leq \frac{2^{n^{3}}+1}{n^{3} 2^{n^{3}-2} \log 2}
$$

* Added on March 14, 1960. 
und die Reihe $\sum_{n=1}^{\infty} H_{G-r_{n}}^{\xi}(1)$ ist konvergent. Für ein genügend grosses $n_{0}$ ist

$$
\sum_{n=n_{0}}^{\infty} H_{G-r_{n}}^{\xi}(1)<1
$$

und (Hilfssatz 4)

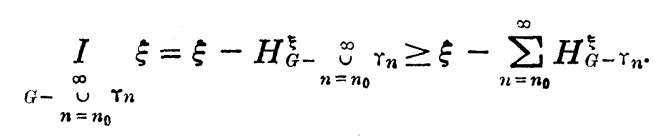

Daraus ergibt sich

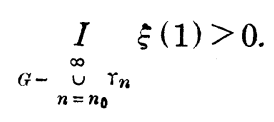

Ist $G_{\xi_{0}}=\left\{\zeta \mid \operatorname{Re} \zeta>\xi_{0}\right\}$, so ist offenbar

$$
\underset{G \xi \xi_{0}}{I} \xi>0
$$

und somit, laut des Folgesatzes 2,

$$
G_{\xi_{0}}-\sum_{n=1}^{I} r_{n} \xi>0 .
$$

Es sei

$$
P(\zeta)=\prod_{k=1}^{\infty}\left(1-\frac{\zeta}{2^{k^{3}}}\right)
$$

und $\sigma_{n}$ das Intervall $\left(2^{n^{3}}+1,2^{(n+1)^{3}}-1\right)$. Wir setzen

$$
\begin{aligned}
& \alpha_{n}=\inf \left\{|P(\xi)| \mid \xi \in \sigma_{n}\right\}, \\
& \beta_{n}=\inf \left\{|P(\zeta)| \mid \zeta \in \partial \gamma_{n}\right\} .
\end{aligned}
$$

Für $\xi \in \sigma_{n}$ ist

$$
\begin{aligned}
& \geq\left[\prod_{k=1}^{n-1}\left(\frac{2^{n^{3}}+1}{2^{k^{3}}}-1\right)\right]\left(\frac{2^{n^{3}}+1}{2^{n^{3}}}-1\right)\left(1-\frac{2^{(n+1)^{3}}-1}{2^{k^{3}}}\right)\left[\prod_{k=n+2}^{\infty}\left(1-\frac{2^{(n+1)^{3}}-1}{2^{k^{3}}}\right)\right] \\
& \geq\left(\frac{2^{n^{3}}}{2^{(n-1)^{3}}}-1\right)^{n-1} \frac{1}{2^{n^{3}}} \frac{1}{2^{(n+1)^{3}}} \prod_{k=n+2}^{\infty}\left(1-\frac{2^{(n+1)^{3}}}{2^{k^{3}}}\right) \\
& \geq\left(2^{3 n^{2-3 n}}\right)^{n-1} \frac{1}{2^{2(n+1)^{3}}} \prod_{k=n+2}^{\infty}\left(1-\frac{1}{2^{k^{3}-(n+1)^{3}}}\right) \geq 2^{n^{3}-12 n^{2}-3 n-2} \prod_{k=1}^{\infty}\left(1-\frac{1}{2^{k}}\right) .
\end{aligned}
$$

Daraus folgt $\lim _{n \rightarrow \infty} \alpha_{n}=\infty$. Für $\zeta \in \partial \gamma_{n}$ ist

$$
|P(\zeta)| \geq\left[\prod_{k=1}^{n-1}\left|1-\frac{\operatorname{Re} \zeta}{2^{k^{3}}}\right|\right] \frac{1}{2^{n^{3}}}\left[\prod_{k=n+1}^{\infty}\left|1-\frac{\operatorname{Re} \zeta}{2^{k^{3}}}\right|\right]
$$




$$
\begin{aligned}
& \geq\left[\prod_{k=1}^{n-1}\left(\frac{2^{n^{3}}-1}{2^{k^{3}}}-1\right)\right] \frac{1}{2^{n^{3}}}\left[\prod_{k=n+1}^{\infty}\left(1-\frac{2^{n^{3}}+1}{2^{k^{3}}}\right)\right] \\
& \geq\left(\frac{2^{n^{3}-1}}{2^{(n-1)^{3}}}-1\right) \frac{1}{2^{n^{3}}} \prod_{k=n+1}^{\infty}\left(1-\frac{1}{2^{k^{3}-n^{3}-1}}\right) \geq 2^{2 n^{3-4} n-1} \prod_{k=1}^{\infty}\left(1-\frac{1}{2^{k}}\right) .
\end{aligned}
$$

Es ist also $\lim _{n \rightarrow \infty} \beta_{n}=\infty$.

Für $\operatorname{Re} \zeta \in \sigma_{n}$ ist

$$
|P(\zeta)| \geq|P(\operatorname{Re} \zeta)| \geq \alpha_{n} .
$$

Für $\zeta \notin \bigcup_{n=1}^{\infty} \gamma_{n}$ und $\xi=R e \zeta \in \gamma_{n}$ ist $|P(\zeta)| \geq \beta_{n}$. Daraus folgt, dass man für jedes $\alpha>0$ ein $\xi_{0}(\alpha)$ finden kann, so dass

$$
P^{-1}(\{|w|<\alpha\}) \cap\left\{\operatorname{Re} \zeta>\xi_{0}(\alpha)\right\} \subset \bigcup_{n=1}^{\infty} \gamma_{n}
$$

ist.

Aus diesen Betrachtungen folgt, dass $\hat{P}$ im Punkte $\zeta=\infty$ definiert und gleich $\infty$ ist, da

$$
\underset{P^{-1}(\mid(|w|>\alpha))}{I} \xi \geq \underset{(^{(y} \xi_{0}(\alpha)-\underbrace{\infty}_{n=1} \gamma_{n}}{I} \xi>0
$$

ist. Setzen wir

$$
f(z)=P\left(\frac{1+z}{1-z}\right)
$$

so ist $\hat{f}$ im Punkte $z=1$ definiert und gleich $\infty$, wogegen $f^{*}$ in demselben Punkte nicht definiert ist. 